\title{
FUNCTIONAL 3-D CELLULOSE AND NITROCELLULOSE PAPER-BASED, MICROFLUIDIC DEVICE UTILIZING ELISA TECHNOLOGY FOR THE DETECTION/DISTINCTION BETWEEN HEMORRHAGIC AND ISCHEMIC STROKES
}

\author{
A Thesis \\ presented to \\ the Faculty of California Polytechnic State University, \\ San Luis Obispo
}

\author{
In Partial Fulfillment \\ of the Requirements for the Degree \\ Master of Science in Biomedical Engineering
}

by

Alicia Leanne Holler

December 2016 
(C)2016

Alicia Leanne Holler ALL RIGHTS RESERVED 


\section{COMMITTEE MEMBERSHIP}

TITLE:

Functional 3-D Cellulose and Nitrocellulose PaperBased, Microfluidic Device Utilizing ELISA Technology for the Detection/Distinction Between Hemorrhagic and Ischemic Strokes

AUTHOR: $\quad$ Alicia Leanne Holler

DATE SUBMITTED: December 2016

COMMITTEE CHAIR: $\quad$ Dr. David Clague, Ph.D.

Associate Professor, Biomedical Engineering California Polytechnic State University

COMMITTEE MEMBER: Dr. Amy Howes, Ph.D.

Lecturer, Biological Sciences

California Polytechnic State University

COMMITTEE MEMBER: Dr. Lily Laiho, Ph.D.

Associate Professor, Biomedical Engineering

California Polytechnic State University 


\begin{abstract}
Functional 3-D Cellulose and Nitrocellulose Paper-Based, Microfluidic Device Utilizing ELISA Technology for the Detection/Distinction Between Hemorrhagic and Ischemic Strokes
\end{abstract}

Alicia Leanne Holler

The purpose of this thesis project is to demonstrate and evaluate an enzyme-linked immunosorbent assay (ELISA) on a paper microfluidic device platform. The integration of ELISA technology onto paper microfluidic chips allows for a quantitative detection of stroke biomarkers, such as glial fibrillary acidic protein (GFAP). Dye experiments were performed to confirm fluid connectivity throughout the 3D chips. Several chip and housing designs were fabricated to determine an optimal design for the microfluidic device. Once this design was finalized, development time testing was performed. The results confirmed that the paper microfluidic device could successfully route fluid throughout its channels at a reasonable rate.

For the biochemistry portion of this thesis project, antibodies were selected to target the intended stroke biomarker: GFAP. However, due to antibody pairing complications, the protein chosen for this project was natural human cardiac troponin $\mathrm{T}$, which is elevated in the bloodstream of patients who have suffered a stroke. Several antibody experiments were performed to help finalize the procedure for performing an ELISA on the paper chip. The final antibody experiment was able to demonstrate that a paper microfluidic device utilizing ELISA techniques can successfully detect a stroke biomarker at physiologically relevant concentrations. Overall, this project supported the ability to accurately and effectively diagnose stroke in a timely manner through the use of a paper microfluidic device. 


\section{ACKNOWLEDGMENTS}

First and foremost I would like to thank my thesis advisor, Dr. David Clague. He was very supportive throughout the entire process and helped make key decisions in our brainstorming sessions. He was a knowledgeable advisor who was able to offer much guidance in this project, especially with the microfluidic concepts explored. He was very passionate about this project and was always hopeful even when we ran into issues; he would quickly make the changes needed to allow for the project to continue. I believe his passion for stroke research helped me realize the impact this project could have on thousands of people who have suffered a stroke.

Secondly, I would like to thank my biology advisor, Dr. Amy Howes. She was an amazing mentor for the biology portion of the project and was so wonderful to work with. She went out of her way to meet me on campus on several weekends to provide me with the reagents needed to perform my experiments. She also never gave up on me as I struggled to finalize my ELISA protocol, but instead comforted me when I didn't get good results and encouraged me to try again. One quote from her that I will always remember is, "Nothing works the first time in science”. This reminded me that many great scientists/engineers have had to push through numerous failures to achieve their successes. For this, I thank Dr. Amy Howes because she kept me motivated to finish my project even when I felt like nothing was going right.

Thirdly, I would like to thank my committee members, Dr. David Clague, Dr. Amy Howes, and Dr. Lily Laiho for taking the time to review my work and attend my defense.

Finally, I would like to thank my family and friends. My parents, Leo and Tracy Holler, have been my biggest supporters throughout my entire college career. Their willingness to support me financially is the only reason I am able to attend Cal Poly and pursue my dreams. 
Furthermore, their endless love has kept me positive through many hard and stressful times in college. They have endured many frantic phone calls from me about how "I will never finish my thesis", but have never lost faith in my ability to finish. For this, I love them more than I can ever express. I would also like to thank all of my close friends and loved ones, especially Nick Phillips, Shannon Durkee, and Amanda Simek, for putting up with my craziness over the past year. They have always been there for me through the ups and downs of this project, listening to me vent when my tests fail or celebrating with me when I finally see good results. I truly have the best support group ever and I love them all to death!

To everyone who has been a part of this process, including Dr. Dave Laiho, Carolyn Teng, and Erik Huynh, thank you so much for all of your help! I would not have been able to accomplish any of this without your support. 


\section{TABLE OF CONTENTS}

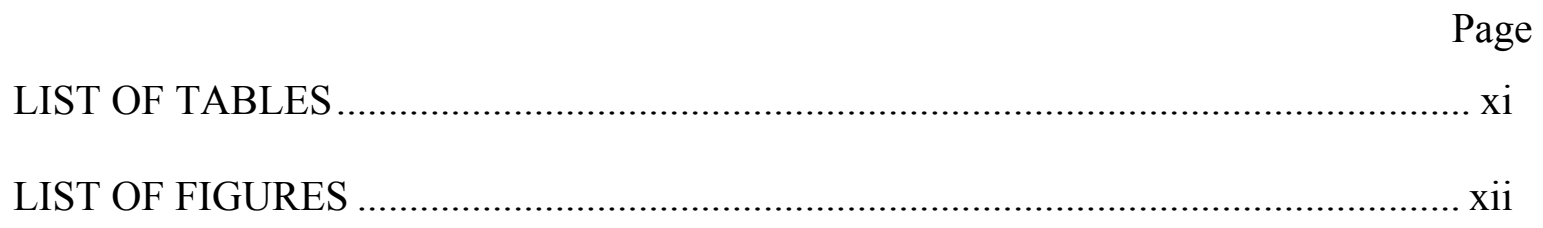

\section{CHAPTER}

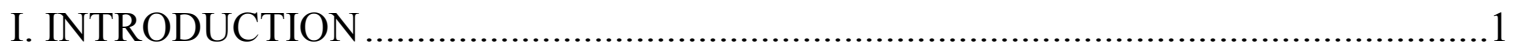

Specific Goals of this Thesis Project and Report.................................................

Background: Ischemic versus Hemorrhagic Stroke............................................2

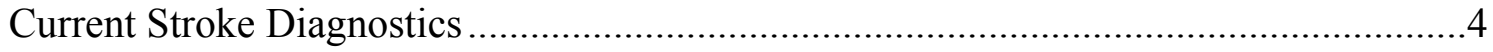

Point-of-Care Diagnostics: A Clinical Need.........................................................6

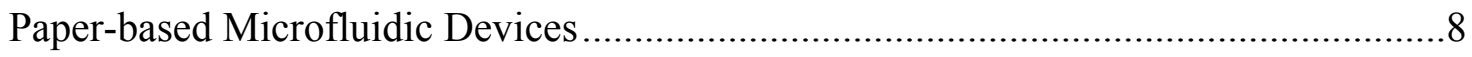

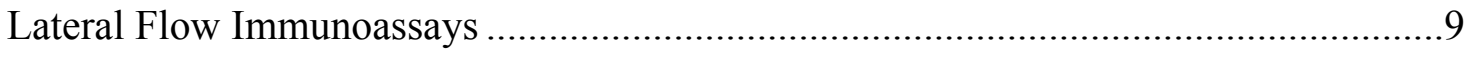

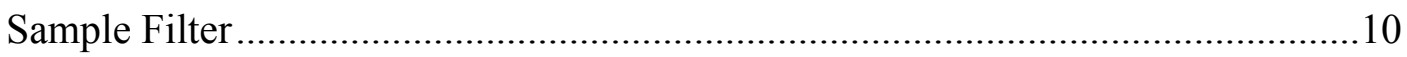

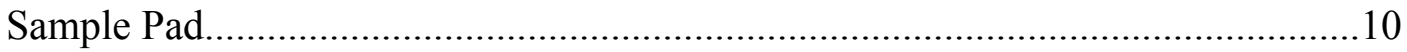

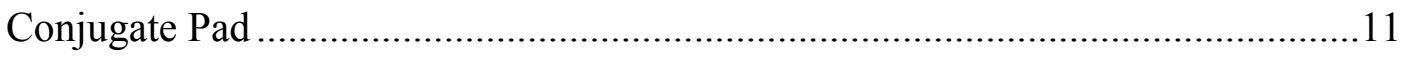

Fluid Routing Membrane .............................................................................. 13

Test and Control Lines.............................................................................. 13

Absorbent Pad ......................................................................................... 14

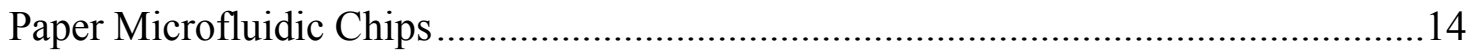

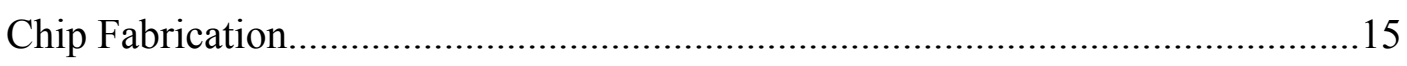

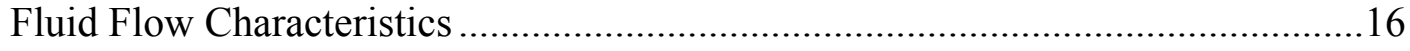

Enzyme-linked Immunosorbent Assay .............................................................. 18

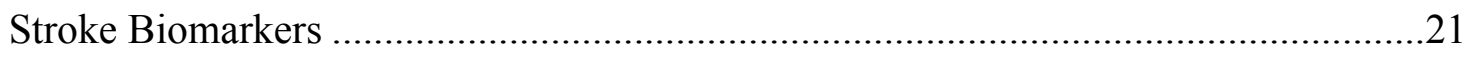

Project Specific Biomarker Selection: GFAP ...........................................................24 


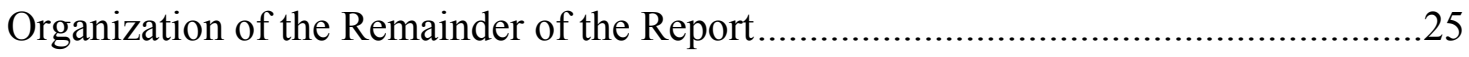

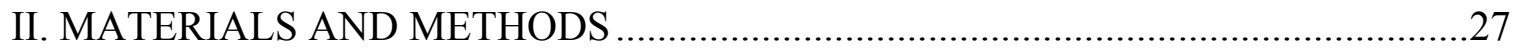

3D Paper Microfluidic Chip Design and Fabrication ............................................27

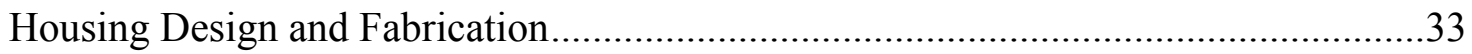

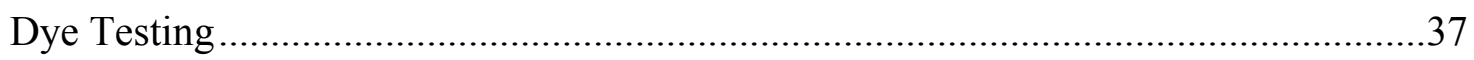

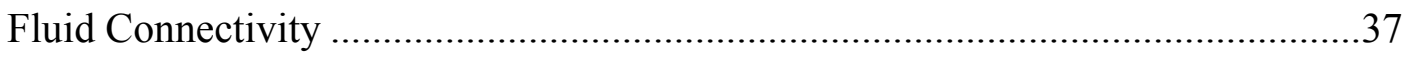

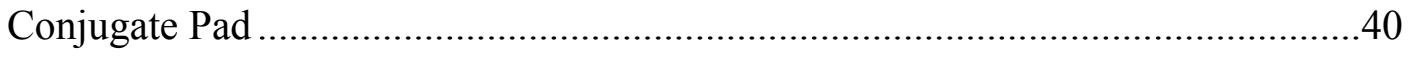

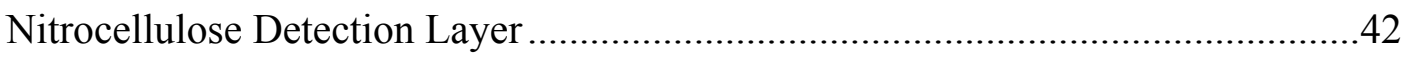

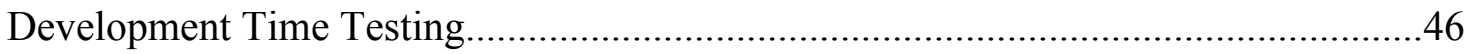

Antibody Experiments: 3D Paper Microfluidic Device........................................48

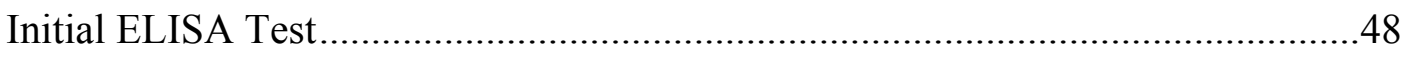

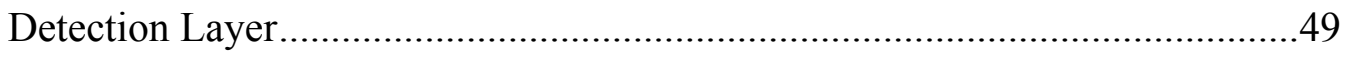

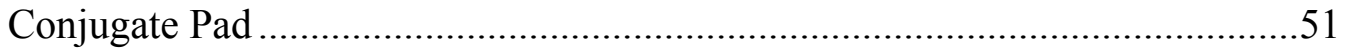

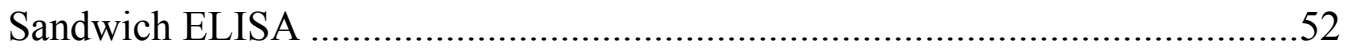

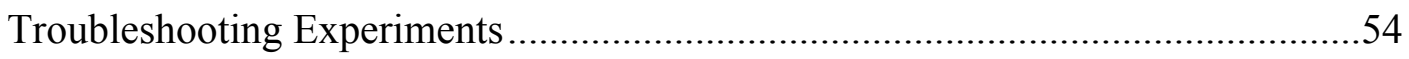

Detection Antibody Storage on Conjugate Pad ............................................54

Inclusion vs. Exclusion of Conjugate Pad …............................................55

BSA Blocking vs. No Blocking ..........................................................57

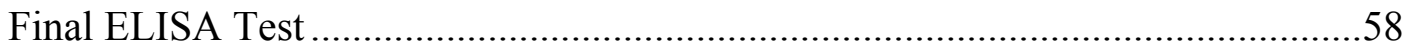

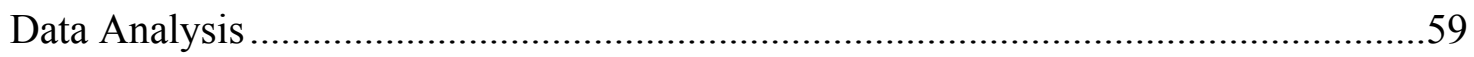

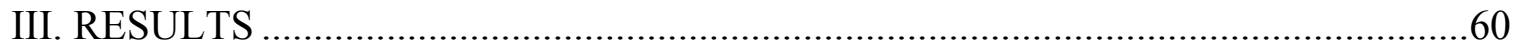

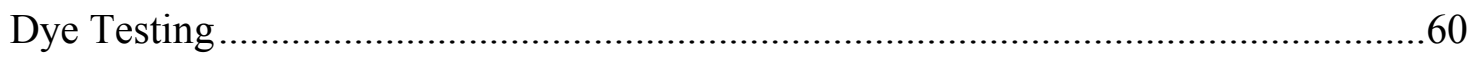

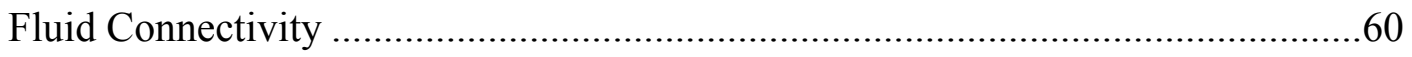


Conjugate Pad

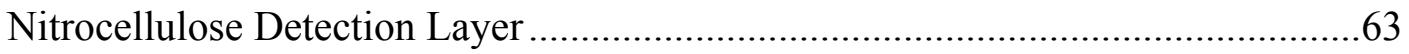

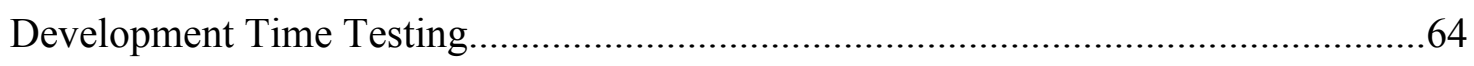

Antibody Experiments: 3D Paper Microfluidic Device..............................................65

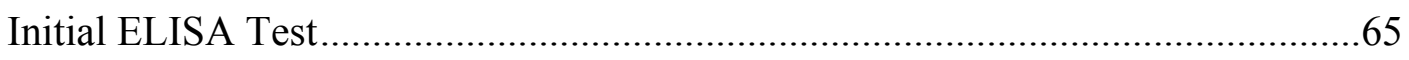

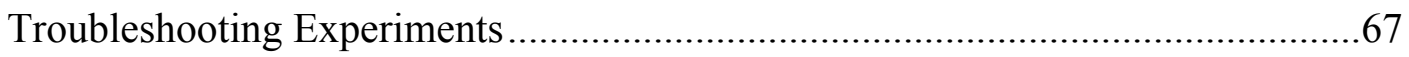

Detection Antibody Storage on Conjugate Pad ...............................................67

Inclusion vs. Exclusion of Conjugate Pad .......................................................69

BSA Blocking vs. No Blocking ....................................................................

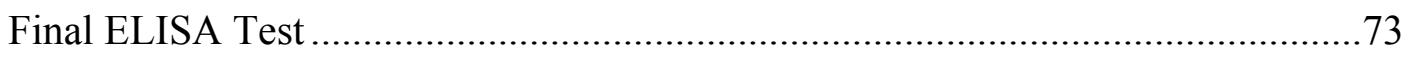

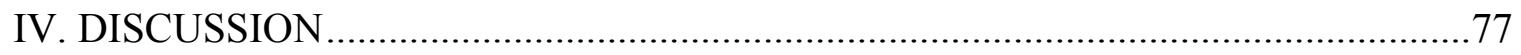

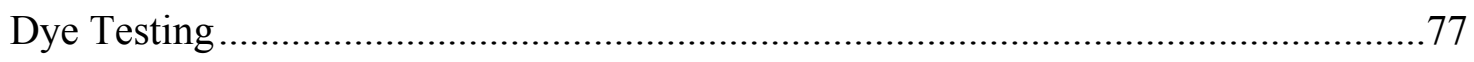

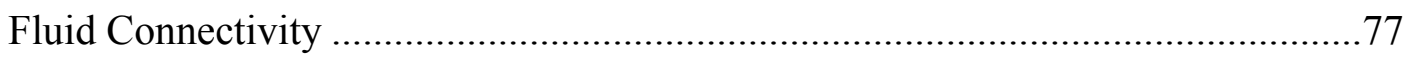

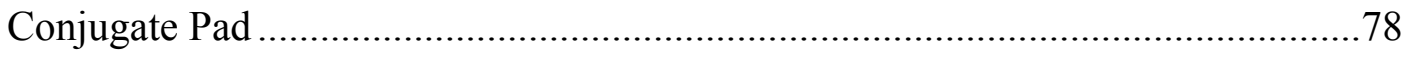

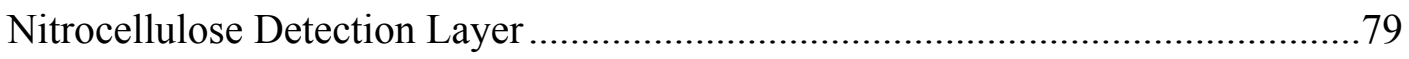

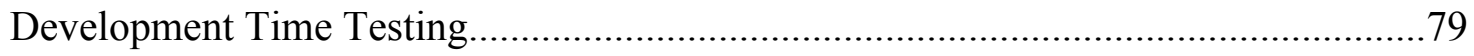

Antibody Experiments: 3D Paper Microfluidic Device.................................................80

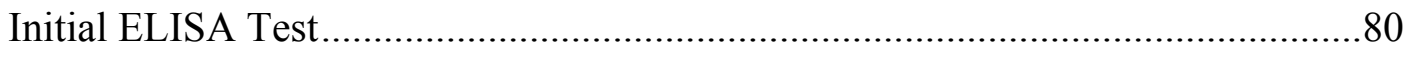

Troubleshooting Experiments ............................................................................. 81

Detection Antibody Storage on Conjugate Pad ................................................... 81

Inclusion vs. Exclusion of Conjugate Pad ....................................................... 82

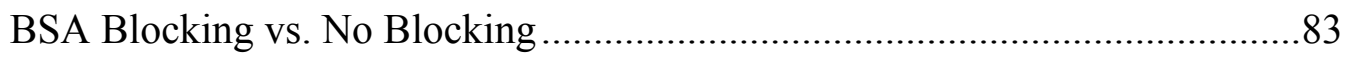

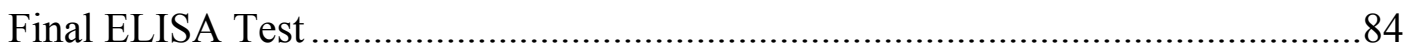


V. CONCLUSIONS.

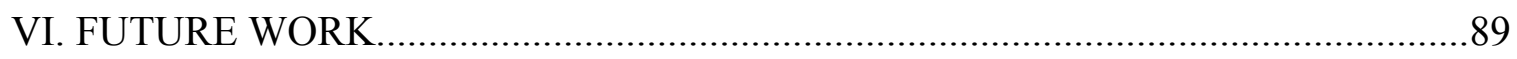

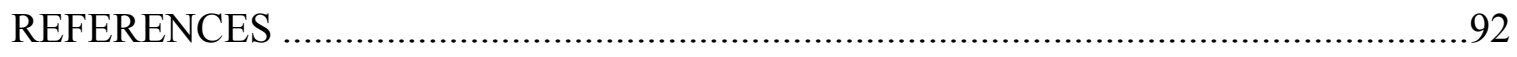

APPENDICES

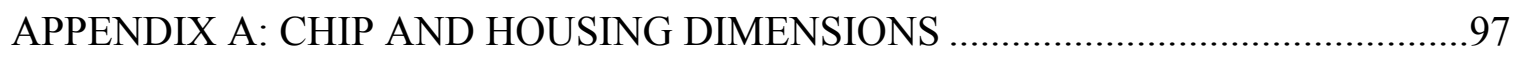

APPENDIX B: BIOCHEMISTRY CALCULATIONS ...........................................99

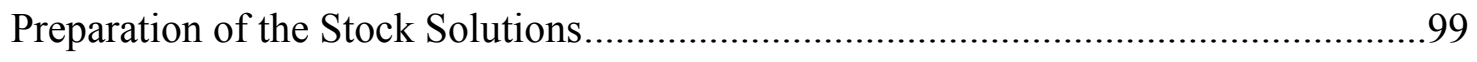

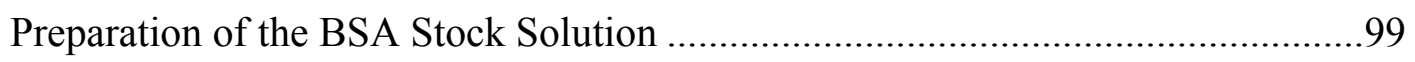

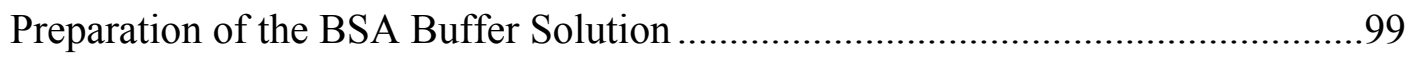

Preparation of the Streptavidin Stock Solution...............................................99

Preparation of the Antibody Stock Solutions........................................................ 100

Preparation of the Capture Antibody Stock Solution ..................................... 100

Preparation of the HRP-conjugate Detection Antibody Stock Solution................100

Minimum Troponin Mass in Stroke Patients ...................................................101

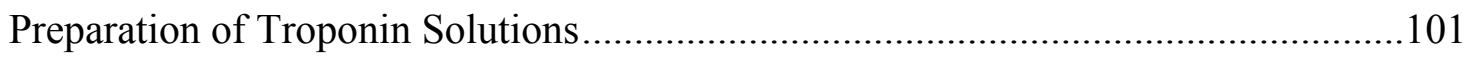

Preparation of $100 \mathrm{ng}$ Troponin Solution Used in the Initial ELISA Test ............101

Preparation of $10 \mathrm{ng}$ Troponin Solution Used in the Initial ELISA Test ..............101

Preparation of $1 \mathrm{ng}$ Troponin Solution Used in the Initial ELISA Test ...............102

Preparation of $1 \mu \mathrm{g}$ Troponin Solution Used in the Final ELISA Test .................102

Preparation of $100 \mathrm{ng}$ Troponin Solution Used in the Final ELISA Test..............103

Preparation of $10 \mathrm{ng}$ Troponin Solution Used in the Final ELISA Test................103 


\section{LIST OF TABLES}

Table

Page

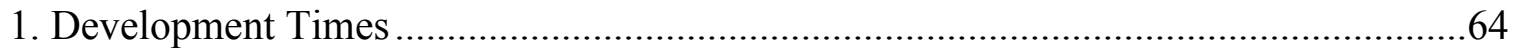

2. Means and Standard Deviations for Development Times ..............................................65 


\section{LIST OF FIGURES}

Figure $\quad$ Page

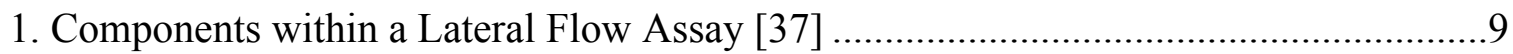

2. Schematic Representation of a Sandwich ELISA [38] ..........................................19

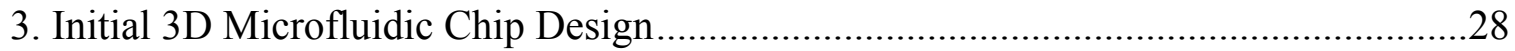

4. Second 3D Microfluidic Chip Design (First Circular Design) ................................. 29

5. Xerox Phaser 8560 Wax Printer Used to Print the Microfluidic Chips........................30

6. Clarkson Digital Hot Plate Used to Melt the Wax on the Microfluidic Chips ..............31

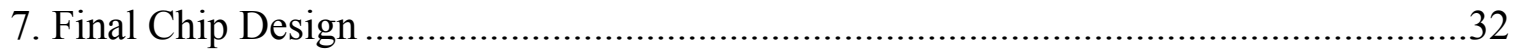

8. Top (left) and Bottom (right) Components of First Housing......................................34

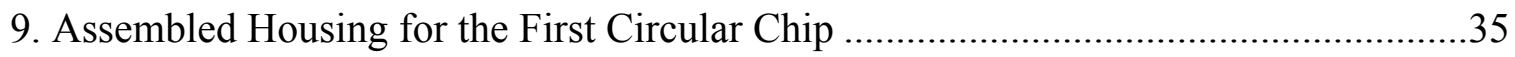

10. Benchtop Press Used to Press the Hex Threaded Inserts into the Housing .................36

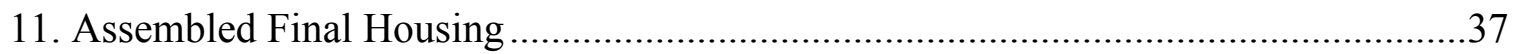

12. First Microfluidic Device Assembled with Screws .................................................38

13. First Microfluidic Device Assembled with Paper Clamps ......................................39

14. Final Microfluidic Device Pictured during Dye Testing .......................................40

15. Dyed Conjugate Pad Made from Cellulose Paper ...................................................41

16. Dyed Fiberglass Conjugate Pad Attached to Top Layer of Chip..............................42

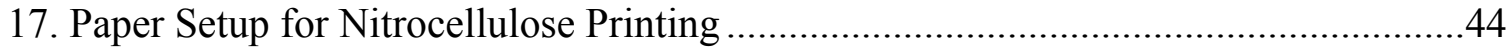

18. Cutting Strategy Used to Remove Nitrocellulose Layer from the Sheet of

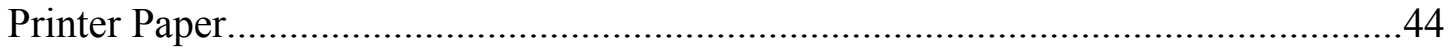

19. Nitrocellulose Detection Layer Taped to the Top Layer of the 3D Chip ...................46

20. Humidity Chamber Attached to Vacuum Pump ....................................................50 
21. BSA Soaking on the Nitrocellulose Chip During the Blocking Step in the ELISA

Procedure

22. Dye Testing Results for First Circular Chip Positioned in the Housing Assembled with Screws 60

23. Dye Testing Results for the First Circular Chip Positioned in the Housing Assembled with Paper Clamps 61

24. Dye Testing Results for the Final Chip Positioned in the Final Housing 61

25. Dye Testing Results for Chip Utilizing the Conjugate Pad Made with Cellulose Paper 62

26. Dye Testing Results for the Chip Utilizing the Conjugate Pad Made with Fiberglass 63

27. Dye Testing Results for the Chip with the Nitrocellulose Layer Included 64

28. Results for First (left) and Second (right) Final Chips Used in the First ELISA Test. 65

29. Quantitative Results for the First Chip Used in the First ELISA Test Obtained Using Custom Matlab Code .66

30. Quantitative Results for the Second Chip Used in the First ELISA Test Obtained Using Custom Matlab Code.

31. Results for Wax-backed (left) and Paper-backed (right) Chips Used in the Detection Antibody Storage on Conjugate Pad Experiment. 68

32. Quantitative Results for Wax-backed (left) and Paper-backed (right) Chips Used in the Detection Antibody Storage on Conjugate Pad Experiment Obtained Using Custom Matlab Code 69 
33. Results for the Inclusion vs. Exclusion of Conjugate Pad Experiment with Channel $\mathrm{C}$ a control, Channels 1 and 2 Excluding the Conjugate Pad, and Channels 3 and

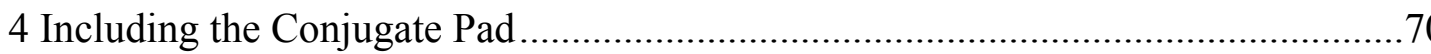

34. Quantitative Results for the Inclusion vs. Exclusion of Conjugate Pad Experiment Obtained Using Custom Matlab Code. Channel C Used as a control, Channels 1 and 2 Excluded the Conjugate Pad, and Channels 3 and 4 Included the Conjugate Pad.

35. Results for the BSA Blocking vs. No Blocking Experiment with Channel C a Control, Channels 1 and 2 Blocked with BSA, and Channels 3 and 4 Unblocked .....72

36. Quantitative Results for BSA Blocking vs. No Blocking Experiment Obtained Using Custom Matlab Code. Channel C Used as a Control, Channels 1 and 2 Blocked with BSA, and Channels 3 and 4 Unblocked

37. Results for the Final ELISA Test with Channel $\mathrm{C}$ a control, Channels 1 and 4 Using $10 \mathrm{ng}$ Troponin, Channels 2 and 5 Using $100 \mathrm{ng}$ Troponin, and Channels 3 and 6 Using $1 \mu \mathrm{g}$ Troponin.

38. Quantitative Results for the Final ELISA Test Obtained Using Custom Matlab Code. Channel C Used as a control, Channels 1 and 4 Using 10 ng Troponin, Channels 2 and 5 Using $100 \mathrm{ng}$ Troponin, and Channels 3 and 6 Using $1 \mu \mathrm{g}$ Troponin...... .75

39. Quantitative Results for the Standard ELISA Test Obtained Using Custom Matlab Code. Well C Used as a Control, Well 1 Using 5 ng Troponin, Well 2 Using 50 ng Troponin, Well 3 Using $500 \mathrm{ng}$ Troponin, and Well 4 Using $1.5 \mu \mathrm{g}$ Troponin 76 
40. Dimensions of the Second Chip Design (First Circular Chip)

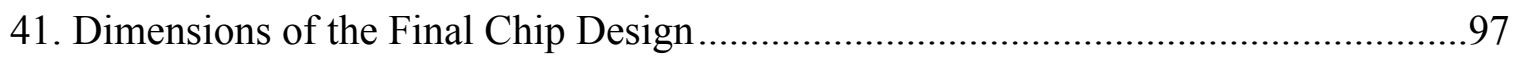

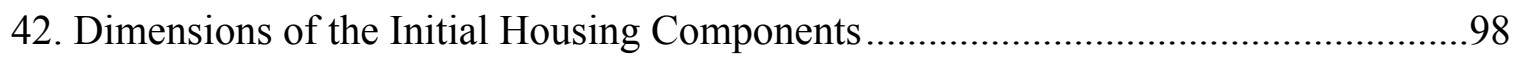

43. Dimensions of the Final Housing Components .................................................... 98 


\section{INTRODUCTION}

\section{Specific Goals of this Thesis Project and Report}

Stroke has a significant impact on the healthcare system in the US and globally. Every year an estimated 15 million people suffer a stroke and deal with issues of immobility, etc. [1]. Accurate, timely, and affordable methods of diagnosing and differentiating different types of stroke remains challenging. For example, CT scans - the current standard diagnostic technique for stroke - do not detect over $80 \%$ of strokes, which often times leads to misdiagnosis [2]. The purpose of this thesis project is to demonstrate and evaluate an enzyme-linked immunosorbent assay (ELISA) on a paper microfluidic device platform. By doing so, we can develop an affordable diagnostic tool that can be used to detect and distinguish between the two major types of stroke: hemorrhagic and ischemic. Furthermore, since many developing countries do not have easy access to imaging equipment, there is a large need for an inexpensive and accessible diagnostic device. Recent innovation in microfluidic paper-based analytical devices has created the potential for a new point-of-care diagnostic tool that is easy to use and accessible. With the discovery of biomarkers that can potentially distinguish between hemorrhagic and ischemic stroke, this paper-based microfluidic device can be designed to target stroke biomarkers and provide a quick, accurate way of diagnosing stroke. By the end of this project, we will have successfully shown that a specific biomarker can be detected on a paper microfluidic chip utilizing ELISA technology. We will accomplish this goal by completing the following:

- Design a full multi-layer paper-chip for ELISA assay

- Design a housing component to apply the appropriate pressure to mediate fluid transfer between layers

- Assemble device in housing and test fluid connectivity via dye tests 
- Redesign chip and housing component and optimize fluid connectivity via dye tests

- Demonstrate ELISA assay on chip

- Quantify the signal to demonstrate a ladder effect using different protein masses

- Compare the results to a standard ELISA

\section{Background: Ischemic versus Hemorrhagic Stroke}

Stroke is a widely studied disease in both the neurological and cardiovascular fields. With it being the third leading cause of death in more industrialized countries, there is a crucial need to improve current diagnostic and treatment methods [3]. To better understand the biological mechanisms for stroke, the disease must be divided into two major types: ischemic and hemorrhagic [3]. Unfortunately, these types of stroke have different underlying causes that lead to similar symptoms, making differentiation of the two difficult.

An ischemic stroke is caused by a lack of blood supply to the brain, often caused by a blockage within an artery that supplies the brain. With reduced blood supply, the brain becomes oxygen-deprived and brain cells begin to die. Cell death is the primary cause of stroke symptoms. An ischemic stroke can be further segmented based on the origin of the blockage or blood clot. The two types of ischemic stroke are thrombotic and embolic. In a thrombotic stroke a blood clot forms in an artery that directly supplies blood to the brain. On the other hand, in an embolic stroke a blockage or blood clot originates elsewhere in the body and travels through the bloodstream to an artery that supplies blood to the brain [4]. However, in both cases, the blockage prevents the brain from receiving an adequate supply of oxygen-rich blood thus leading to ischemia. 
With the reduction of oxygen delivery in an ischemic stroke, the brain cells are unable to produce adenosine triphosphate (ATP). This prevents ATP-dependent ion pumps from correctly pumping $\mathrm{Na}^{+}$across brain cell membranes, leading to an altered $\mathrm{Na}^{+}$concentration gradient [5]. This concentration gradient leads to altered behavior in the glutamate transporters, which then releases an excessive amount of glutamate in the extracellular space.. Increase in extracellular glutamate causes an increased inflow of $\mathrm{Ca}^{2+}$ via NMDA, AMPA, and kainate receptors. The influx of $\mathrm{Ca}^{2+}$ eventually leads to high intracellular levels of $\mathrm{Ca}^{2+}$ that causes cell death by necrosis and apoptosis [5]. This irreversible brain damage leads to a loss of function in many areas of the brain, causing symptoms such as weakness and/or numbness on one side of the body, speech, vision, and cognitive impairment, and a severe headache.

Hemorrhagic stroke affects the brain in a different manner than ischemic. In a hemorrhagic stroke, a blood vessel in the brain leaks or bursts causing a pooling of blood in the brain. There are two types of hemorrhagic stroke that can be differentiated based on the location of the burst blood vessel. An intracerebral hemorrhagic stroke occurs when a blood vessel inside the brain bursts. A subarachnoid hemorrhagic stroke occurs when a blood vessel on the surface of the brain bursts and the blood pools between the inner and middle layers of the membrane that covers the brain [4]. Both types lead to an accumulation of blood in or around the brain, which causes it to swell. This immediate loss of blood causes ischemia in the brain. As the brain swells it also pushes against the skull and intracranial pressure increases drastically. This increased pressure causes damage to the brain tissue and may also lead to ischemia if the pressure is large enough to cut off blood supply [5].

Overall, an ischemic stroke is caused by a blockage of the blood supply to the brain, while a hemorrhagic stroke is caused by an accumulation of blood in the brain due to a burst 
blood vessel. These two conditions require different treatments, thus differentiation between the two is crucial during diagnosis. The most effective treatment for ischemic stroke is thrombolytic therapy, in which drugs are administered to break up blood clots. The only FDA approved thrombolytic drug for ischemic stroke is tissue plasminogen activator (tPA). However, for this drug to be effective it must be administered within 3 hours of symptom onset [6]. This makes the diagnosis of stroke a time sensitive process. However, if tPA is accidently administered to a patient with hemorrhagic stroke their condition will likely worsen since clotting prevents further hemorrhage. Therefore, tPA will exacerbate the injury by increasing the rate of hemorrhage and causing an even larger accumulation of blood in the brain. Thus, to treat stroke patients effectively and in a timely manner, a quick and accurate way of distinguishing between the two types of strokes is needed. Unfortunately, these types of stroke display almost identical clinical symptoms since they both ultimately lead to brain cell damage. A large area of research is aimed toward improving the differentiation between ischemic and hemorrhagic stroke during the diagnostic process.

\section{Current Stroke Diagnostics}

There are currently few techniques used to diagnose a stroke. When a patient comes in showing symptoms of a stroke, they are first examined by a doctor. The doctor then decides if further testing is necessary based on their subjective opinion on whether or not they believe the patient has had a stroke. Unfortunately, strokes are often confused with hypoglycemia, inner ear infection, and seizures since they have similar symptoms. Although recent statistics have shown that $92 \%$ of stroke cases are properly diagnosed by a primary care physician, this only accounts for doctors who are regularly exposed to stroke victims [3]. However, doctors who are less familiar with the condition may be less confident in their diagnosis causing this number to drop. 
This subgroup of less-experienced physicians often misdiagnose stroke and send patients home without further investigation of their condition. This leads to a delayed diagnosis, which can lead to more severe brain damage and other complications. Furthermore, even when a stroke is diagnosed accurately the physician must then differentiate between the two major types of stroke to effectively treat the patient. Currently, the only method available for differentiating between ischemic and hemorrhagic stroke is neuroimaging.

The two most commonly used imaging technologies for the diagnosis of stroke are computed tomography (CT) and magnetic resonance imaging (MRI). However, $\mathrm{CT}$ is known to have a wider accessibility and shorter processing time than MRI. Due to this, CT is the most common imaging diagnostic used in the ER today [7]. However, CT scans are not as effective for the detection of ischemic strokes due to their insensitivity toward early ischemia. Since ischemic strokes make up about $87 \%$ of all stroke cases, detection of ischemia is crucial in the diagnostic process [8]. MRI diffusion-weighted imaging (DWI) has proven to be much more sensitive in diagnosing stroke in the first few hours than noncontrast CT (NCCT), with sensitivity levels of $95 \%-100 \%$ compared to $42 \%-75 \%$, respectively [3]. However, MRI scanners are expensive and thus not as widely available. Furthermore, the MRI scan takes about 15-20 minutes, which is not ideal for diagnosing such a time sensitive condition [3]. Since there is such a small window of opportunity for effective treatment with tPA, every minute in the diagnostic process counts.

Although neuroimaging is currently the standard diagnostic technique for stroke, there are many drawbacks to using these imaging technologies. First, it may take a few hours for the stroke-affected area of the brain to show any abnormalities on a CT scan. This would mean that CT images may not be reliable within the first few hours of symptom onset. Unfortunately, 
those first few hours are the most crucial point in the diagnostic process since ischemic stroke can only be treated with tPA in the first 3 hours [6]. Furthermore, the contrast solution used in magnetic resonance angiography (MRA), the most common type of MRI, can cause complications in patients with kidney problems. This contrast solution is known to have detrimental effects on kidney health [9]. Additionally, if the stroke-affected area in the brain is too small or in a position that is difficult to image, the physician may misdiagnose the patient due to an inaccurate interpretation of the MRI or CT images [10]. Overall, the current diagnostic process for stroke is inefficient due to time constraints, inaccessibility of equipment, and the dependence on physicians to accurately interpret the results of CT or MRI scans.

\section{Point-of-Care Diagnostics: A Clinical Need}

Point-of-care (POC) technology is shaping the future of diagnostics across the world. With an increasing need for quick and easy-to-use diagnostic tools, researchers continue to improve and develop POC devices. These devices are intended to be used at the site of care, whether it be at home, in a disaster response, in low-income healthcare systems, or in the field of battle for military forces. POC technology can be administered by a single person in almost any location, unlike MRI and CT scanners that require the laboratory staff or a dedicated facility to produce results. The user can follow simple instructions with little training to perform the test and the results will be displayed within seconds to hours. Typically, these devices require input of some bodily fluid, such as blood, urine, or saliva, and target a particular protein, metabolite, human cell, etc. within that fluid [11]. Interpretation of the result is also simple as it is commonly displayed with a color change or stripe on the device.

There are many advantages to using POC technology -- these easy-to-use diagnostic tools save both time and money. Instead of traveling to a dedicated imaging clinic with the necessary 
equipment, patients or physicians can perform diagnostic tests on the spot in almost any location since the devices can easily fit in a backpack or hand bag. This is a huge benefit in low-resource settings where expensive medical equipment is difficult to access. Recent statistics show that the incidence of stroke in developing countries is increasing rapidly and it is predicted that stroke mortality will triple in the next two decades in Latin America, the Middle East, and sub-Saharan Africa [1]. This highlights the immediate need for low-cost and accessible diagnostic tools. Furthermore, the results of POC devices do not need to be sent to a laboratory or specialist for interpretation, but can instead be read directly by the user at the site of care, allowing nonmedical users to use the diagnostic device and reliably interpret the results. Additionally, as more POC devices are intended to be used by patients at home, researchers are developing ways for physicians to monitor this self-testing process and access the data collected. This helps ensure that patients are testing themselves correctly and allows for a double assessment of the results by both the patient and their physician through telecommunication [11]. Also, by allowing patients to perform these diagnostic tests at home they no longer have to worry about the cost of traveling to a clinic. The POC devices are typically low-cost and medical facilities are able to reduce operating expenses by admitting fewer patients and reducing use or procurement of expensive medical machinery, such as MRI scanners. Furthermore, POC devices do not require power to operate, allowing them to be used in places where electricity may be unreliable.

Many current POC devices utilize microfluidic techniques to function. As the field of microfluidics advances it has become clear that microfluidic chips make a great platform for these POC devices. Their small size and relatively fast wicking times make these chips ideal for portable diagnostic tools. Furthermore, since the samples are transported at the microscopic 
level, small amounts of sample and reagents are needed. For these reasons, research involving the use of microfluidics in POC devices has increased drastically over the past few years [12].

\section{Paper-based Microfluidic Devices}

Microfluidic paper-based analytical devices ( $\mu$ PADs) are an emerging form of POC technology that are designed to be used as diagnostic tools in low-resource settings. According to the World Health Organization, POC diagnostic devices "should be ASSURED: affordable, sensitive, specific, user-friendly, rapid and robust, equipment free and deliverable to end-users" [13]. Although these ASSURED diagnostic assays can have different types of platforms, a new common material being used is paper. The devices utilizing this new platform are known as microfluidic paper-based analytical devices ( $\mu$ PADs). These devices require only small amounts of sample since the fluid moves through the device on a microscopic level. Furthermore, since $\mu$ PADs utilize microfluidic techniques, there is no need for external supporting equipment since the fluid is capable of moving through the device on its own due to capillarity and evaporation.

Due to the wide availability of paper, these devices can typically be made for less than one cent, which is affordable even in developing countries [13]. Aside from being inexpensive, paper has several other advantages: it is lightweight, flexible, and compatible with biological samples, modifiable to house a variety of functional groups, light-colored allowing for high contrast with any color changes, and flammable allowing for safe disposal after use.

Furthermore, paper can be engineered into a variety of forms with varying properties such as pore size and the presence of additional fibers or polymers. This allows researchers to select a specific paper type based on the properties they desire for a particular application [13]. Overall, paper platforms allow for the development of a wide variety of paper-based microfluidic devices. One common form of these paper-based diagnostic assays is a lateral-flow immunoassay. 


\section{Lateral Flow Immunoassays}

The lateral-flow immunoassay is the most common assay integrated into POC devices today. Some of the many applications of this assay include pregnancy confirmation, drug and infectious disease screening, and detection of protein markers that may aid in the diagnosis of numerous conditions such as stroke and heart attack [11]. Lateral flow assays are made up of different components that all play an important role in the detection process of the device. These components are a sample filter, sample pad, conjugate pad, test and control lines, and absorbent pad. Each of these components will be discussed in detail in the following sections. Figure 1 illustrates these components, with the exception of the sample filter.

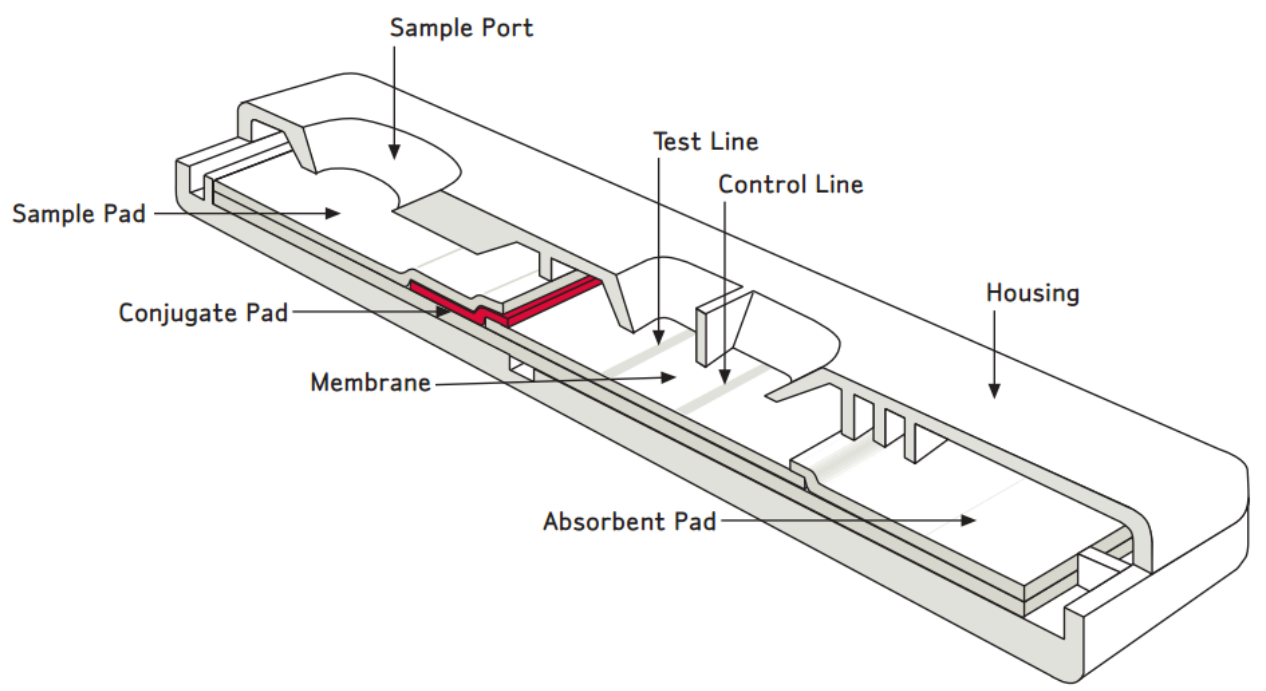

Figure 1: Components within a Lateral Flow Assay [37]

Each component relies on porous membranes for the transportation of a sample throughout the device. As the sample is wicked through the assay it is exposed to antibodies or nucleic acid fragments which are used to target and capture a particular analyte, which is the substance being identified or measured. If the targeted analyte is present in the sample, the device will signal a positive result with either a color change or stripe once a substrate is added 
[14]. This detection process requires each section of a lateral-flow assay to be prepared with specific materials and reagents.

\section{Sample Filter}

A sample, such as blood, must be properly filtered to remove any unwanted components that may affect the detection process of the lateral flow assay before it is applied to the device. Many biological samples require filtration to prepare and sterilize the sample to ensure that impurities, micro-organisms, and unnecessary cells are removed before being exposed to the device. For example, when dealing with whole blood samples, red blood cells (RBC) must be filtered out to isolate the plasma portion of the sample. Filtration is a crucial step for POC devices because RBCs typically have larger diameters than the membrane pores of the lateral flow assay, which causes the RBCs to clog the membrane and prevents the sample from flowing through the assay. Furthermore, RBCs may also hemolyse making the entire assay unusable [15]. Some common biological sample filters used are polyethersulfone (PES) and polyvinylidene fluoride (PVDF). These filters are favorable because they have low proteinbinding affinity, which allows for the filter to catch unwanted particles while still allowing proteins to flow through [16]. Since lateral flow assays often target proteins, allowing for proteins to remain within the sample is critical. Once the sample is properly filtered, it is pipetted onto the device.

\section{$\underline{\text { Sample Pad }}$}

The filtered sample is introduced to the lateral flow assay by directly dropping it onto the sample pad. The main purpose of the sample pad is to spread the sample evenly before it enters the conjugate pad, controlling the flow of the sample to the conjugate pad so the sample does not over saturate the detection reagent. Proteins and surfactants may be added to the sample pad to 
help resolubilize the detection reagent that is dried on the conjugate pad, as well as reduce nonspecific binding of the detection reagent and analyte to other materials in the lateral flow assay. The components that are typically added to this pad are the protein albumin and surfactants SDS or Tween 20 [14]. By adding a blocking agent to the sample pad, such as albumin, the membrane downstream from the pad will also be successfully blocked. This prevents the antibodies in the assay from binding to non-specific proteins present due to charge attraction. The blocking agent blocks the charge of all the proteins in the sample so that the antibodies will only bind to the target analyte [17]. Lastly, components, such as buffer salts, may be added to the sample pad to alter the chemical nature of the sample. This alteration may be necessary to control the $\mathrm{pH}$ or ionic strength of the sample to ensure that it will react with detection and capture reagents appropriately [14].

The material for the sample pad needs to be capable of holding and dispersing large volumes of fluid. The two common materials used are woven meshes and cellulose filters. While woven meshes can evenly disperse the sample, they are unable to hold large volumes. Thus, cellulose filters are more commonly used since they have a much larger bed volume and are able to hold large amounts of sample and added components [14]. However, cellulose filters are extremely brittle and must be handled with great care.

\section{Conjugate Pad}

After wicking through the sample pad, the sample is directed onto the conjugate pad. The main purpose of the conjugate pad is to hold the detection reagent and introduce the sample as it flows through the pad. If the analyte is present in the sample, the detection reagent will bind to the analyte and form an immunocomplex that will be detected further downstream at the test line. The conjugate pad also controls the flow of the detection reagent and sample such that they 
flow through the conjugate pad and onto the fluid routing membrane at a similar rate. As the sample moves through the conjugate pad, the detection reagent is solubilized and carried with the sample fluid. The detection reagent consists of an antibody specific to the target analyte attached to a detector agent, which generates a signal when a substrate is later added to the assay. Some common detector agents used are: colloidal-gold, silver enhanced gold, blue latex bead, carbon black nanoparticles, and horseradish peroxidase [18].

To maximize detection of the target analyte, there needs to be minimal non-specific binding of the detection reagent or analyte to the conjugate pad. The blocking agents from the sample pad should wick throughout the conjugate pad and prevent this from occurring. The detection reagent and sample must migrate toward the test line in a uniform flow. As long as the analyte in the sample reaches the test line before or at the same time as the detection reagent, it can bind to both the capture and detection reagents and contribute to the detection signal. This signal is generated by the interaction between the HRP conjugated to the detection reagent and the 3,3',5,5'-Tetramethylbenzidine (TMB) substrate later added to the device. However, if the sample moves at a slower rate than the detection reagent, the analyte will reach the test line after the detection reagent has already passed. This will prevent the analyte from binding to the detection reagent, which means the analyte will not contribute to the detection signal since the detector agent is part of the detection reagent. To ensure the detection reagent and analyte flow through the membrane at the same rate, the detection reagent should be similar in size to the analyte, as smaller molecules travel faster in fluid. Therefore, a consistent molecular size between the detection reagent and analyte is desired to ensure the particles reach the test line at the same time [19]. The materials commonly used for the conjugate pad are non-woven filters. 
These filters need to have low non-specific binding and be able to hold large volumes. The filters can be made up of glass fiber, cellulose, surface-treated polyester, or polypropylene [14].

\section{Fluid Routing Membrane}

For the sample to travel from the conjugate pad to the test and control lines, it needs to flow through a fluid routing membrane. For the proteins in the sample to travel with little interference, this routing membrane must have low protein-binding affinity. The most common membrane used for this fluid routing layer is cellulose acetate. Cellulose acetate membrane filters are composed of cellulose di- and triacetate and exhibit low static charge. For this reason, the filter has low protein-binding affinity because it has little attraction toward the dipole moment of proteins [20].

\section{$\underline{\text { Test and Control Lines }}$}

The test and control lines are integrated into the fluid routing membrane to catch and hold the target analyte or detection antibody. The test line consists of immobilized capture antibodies, which bind to the target analyte as it flows over the line, sandwiching the target analyte between the capture and detection antibodies while holding it in one place. Capturing the target analyte this way allows for the generated signal to be concentrated in one area on the device, making it easier to observe and analyze. The control line is further downstream from the test line and consists of a line of immobilized antibodies targeting the detection antibody [21]. These antibodies bind the detection antibody and also hold it in one place. This line is used to verify that the sample has successfully made it to the detection region. Once the fluid has wicked over both lines, unbound detection antibody will bind to the control line and generate a signal after the addition of TMB substrate. This signal ensures that the sample was successfully exposed to both the test and control lines. If the test line does not generate a signal, the user can assume that the 
target analyte is not present in the solution. However, if the control line does not generate a signal, this may indicate that either the fluid was unable to reach the test and control lines or there was not a sufficient concentration of detection antibody in the conjugate pad to generate a signal. With the lack of signal at the control line, the test results are unreliable and a new test should be performed with a larger sample volume.

For the test and control lines to be able to hold the capture and control antibodies, the membrane must have high protein-binding affinity. One common material used for these detection areas is nitrocellulose. The strong dipole of the nitrate ester within nitrocellulose is highly attracted to the dipole of peptide bonds in proteins, forming a strong bond between proteins and the nitrocellulose membrane [14].

\section{$\underline{\text { Absorbent Pad }}$}

Once the fluid travels past the test and control lines, it is directed toward the absorbent pad. The main purpose of this pad is to provide a large bed volume where the fluid can be absorbed. This allows for larger sample volumes since the pad provides a place for the fluid to accumulate, allowing for additional fluid to continue wicking through the device. Without the absorbent pad, the bed volume of the membrane would quickly be filled and no additional fluid would be able to flow. The most common material used for these pads are cellulose fibers. The thickness and length of the absorbent pad can be designed to accommodate for a specific sample volume [14].

\section{Paper Microfluidic Chips}

To serve as a platform for microfluidic POC devices, the lateral flow assay can be miniaturized into a chip format. In this format the assay can be separated into many channels that are isolated from one another, which allows for multiple tests to be performed at once. 
Although the chip contains all of the same components as a lateral flow assay, the layers are stacked on top of one another instead of laid laterally in a row. This requires the fluid to flow vertically and laterally through the chip. For the fluid to stay within specific channels, there must be barriers within the chip to direct the fluid in the appropriate directions and prevent crosscontamination between channels.

\section{Chip Fabrication}

Microfluidic chips can be manufactured using various methods to ensure separation of these channels. In general, the channels should be composed of a hydrophilic material with the surrounding areas being covered in a hydrophobic material. These hydrophobic barriers prevent the fluid from flowing outside of the hydrophilic channels and thus direct fluid flow. To fabricate hydrophobic regions, various techniques have been used, including photolithography, plotting method with hexanes-dissolved PDMS, plasma treatment, inkjet printing, and wax printing [22].

For the purposes of this thesis, the wax printing method is used to fabricate the microfluidic chip, as it has become the gold standard for patterning paper in the chip fabrication process. The wax printing method is simple and relatively fast since it only requires printing wax onto paper and baking it until the wax melts through. The process can be performed in 5-10 minutes. Furthermore, wax printing is low cost and environmentally friendly because both wax and paper are cheap and disposable through burning since no organic compounds are present. Since the wax is hydrophobic, it is printed in the areas surrounding the designated channels to keep fluid flow within the channels.

Once the channel design is created in a mapping software such as AutoCAD, the design can be printed using a wax printer. The cellulose or nitrocellulose paper is fed into the printer 
and the wax is printed on in the designed pattern. Then, the wax-printed paper is placed either in an oven or on a hot plate at a certain temperature for about 5 minutes to allow the wax to melt all the way through the paper. The paper microfluidic chip is now ready for fluid entry [22].

\section{$\underline{\text { Fluid Flow Characteristics }}$}

There are a few governing equations in microfluidics that explain how fluid flows through a microfluidic chip. The first important equation involves the Reynold's number, a dimensionless parameter that is defined as the ratio of inertial forces to viscous forces. The Reynold's number helps to predict what the dominant forces are that move a fluid through a particular medium [23]. The Reynold's number can be expressed as follows:

$$
\mathrm{R}_{\mathrm{e}}=\left(\rho \mathrm{VD}_{\mathrm{H}}\right) / \eta
$$

In this equation, $\rho$ is the fluid density $\left(\mathrm{kg} / \mathrm{m}^{3}\right), \mathrm{V}$ is the fluid velocity $(\mathrm{m} / \mathrm{s}), \mathrm{D}_{\mathrm{H}}$ is the hydraulic diameter of the channel $(\mathrm{m})$, and $\eta$ is the fluid viscosity $(\mathrm{kg} /(\mathrm{m} * \mathrm{~s}))$ [24]. The equation for the hydraulic diameter can be found in Equation 2.

$$
\mathrm{D}_{\mathrm{H}}=2 \mathrm{ab} /(\mathrm{a}+\mathrm{b})
$$

In this equation, $a$ is the width of the channel and $b$ is the height of channel [24]. The Reynold's number for fluids in microfluidics is typically much smaller than one. When the Reynold's number is this small, the inertial forces can be disregarded and the flow can be considered laminar. This impacts another governing equation in microfluidics known as the Navier-Stokes equation. The Navier-Stokes equation for an incompressible fluid, e.g. blood, can be found in Equation 3. 


$$
\rho_{\infty}[(\mathrm{d} / \mathrm{dt}) \mathrm{v}+(\mathrm{v} \cdot \nabla) \mathrm{v}]=-\nabla \mathrm{P}+\eta \nabla^{2} \mathrm{v}+\rho_{\infty} \mathrm{g}
$$

In this equation, $\rho_{\infty}$ is the fluid density $\left(\mathrm{kg} / \mathrm{m}^{3}\right), \mathrm{v}$ is the fluid velocity $(\mathrm{m} / \mathrm{s}), \mathrm{P}$ is the pressure $\left(\mathrm{N} / \mathrm{m}^{2}\right), \eta$ is the fluid viscosity $(\mathrm{kg} / \mathrm{s} \cdot \mathrm{m})$, and $\mathrm{g}$ is gravitational acceleration $\left(\mathrm{m} / \mathrm{s}^{2}\right)[25]$. Since a low Reynold's number indicates that viscous forces have a much larger effect than inertial forces on fluid flow, the inertial term on the left side of the Navier-Stokes equation can be ignored. When dealing with microfluidics in which no external forces are present and $\mathrm{R}_{\mathrm{e}}<<1$, this leaves only the viscous and pressure terms in the equation. The Navier-Stokes equation reduces to the Stokes equation:

$$
0=-\nabla P+\eta\left(\nabla^{2}\right) v
$$

This linear equation can be used to describe the typical flow seen in microfluidic chips, known as Poiseuille flow. Poiseuille flow is characterized by pressure-driven, steady state flow through a straight channel. In this type of flow, the fluid is subject to a non-slip boundary condition on the surface. Also, the area of the channel downstream from the fluid has a lower pressure than the area where fluid is currently flowing through, creating a pressure gradient. This pressure gradient is the driving force behind fluid flow [26]. The flow through a porous medium, such as cellulose paper, can be described by Darcy's Law [27].

$$
V=-\frac{K}{\eta} \Delta P
$$

In this equation, $\mathrm{K}$ is the medium permeability $\left(\mathrm{m}^{2}\right), \Delta \mathrm{P}$ is the pressure gradient within the channel, and $\eta$ is the fluid viscosity $(\mathrm{kg} / \mathrm{s} \cdot \mathrm{m})$. In a porous medium, the pressure gradient is a consequence of capillary action. Due to a balance between the forces that spread a liquid and the 
surface tension that minimizes the interfacial area, the liquid-gas interface in a porous medium is curved [28]. The pressure of the concave side of the interface is greater than the convex side. The Young-Laplace equation can be used to describe this pressure difference [28].

$$
\nabla P=\lambda\left(1 / \mathrm{R}_{1}+1 / \mathrm{R}_{2}\right)
$$

In this equation, $\lambda$ is the surface tension of the fluid $(\mathrm{N} / \mathrm{m})$ and $\mathrm{R}_{1}$ and $\mathrm{R}_{2}$ are principal radii of curvature of the fluid $(\mathrm{m})$. Once this pressure difference is known, the volumetric flow rate through the paper channels can be calculated using the following equation [27].

$$
Q=-\frac{K w h}{\eta L} \nabla P
$$

In this equation, $\mathrm{w}$ is the width of the channel $(\mathrm{m}), \mathrm{h}$ is the thickness of the channel (m), and $\mathrm{L}$ is the length of the channel (m). These governing equations rely on both the width and length of a rectangular channel within a microfluidic chip. For this reason, the fluid flow characteristics on a chip can be controlled by altering the dimensions of the channels. The width component depends on both the membrane pore size as well as the width of the channel. Either one of these properties can be changed to control fluid flow rate. Furthermore, the length of a channel can be altered to increase or decrease fluid flow. Based on Equation 6, fluid flow is much slower in longer channels. Overall, the channel dimensions on a microfluidic chip have a large impact on fluid flow characteristics. This is important in POC devices because these characteristics ultimately determine the development time of the lateral flow assay on the chip.

\section{Enzyme-linked Immunosorbent Assay}

Another assay that uses antibodies and a color change to identify if a particular analyte is present in a sample is the enzyme-linked immunosorbent assay (ELISA). This assay is similar to 
a lateral flow assay except that it is typically performed in wells instead of on paper. The two most common types of ELISA are a sandwich ELISA and a competitive ELISA. Both have a similar end result consisting of a series of wells with varying color intensity depending on how much of the target analyte is present in each sample. A higher concentration of analyte present creates a more intense color change. A schematic representation of a sandwich ELISA can be seen in Figure 2.

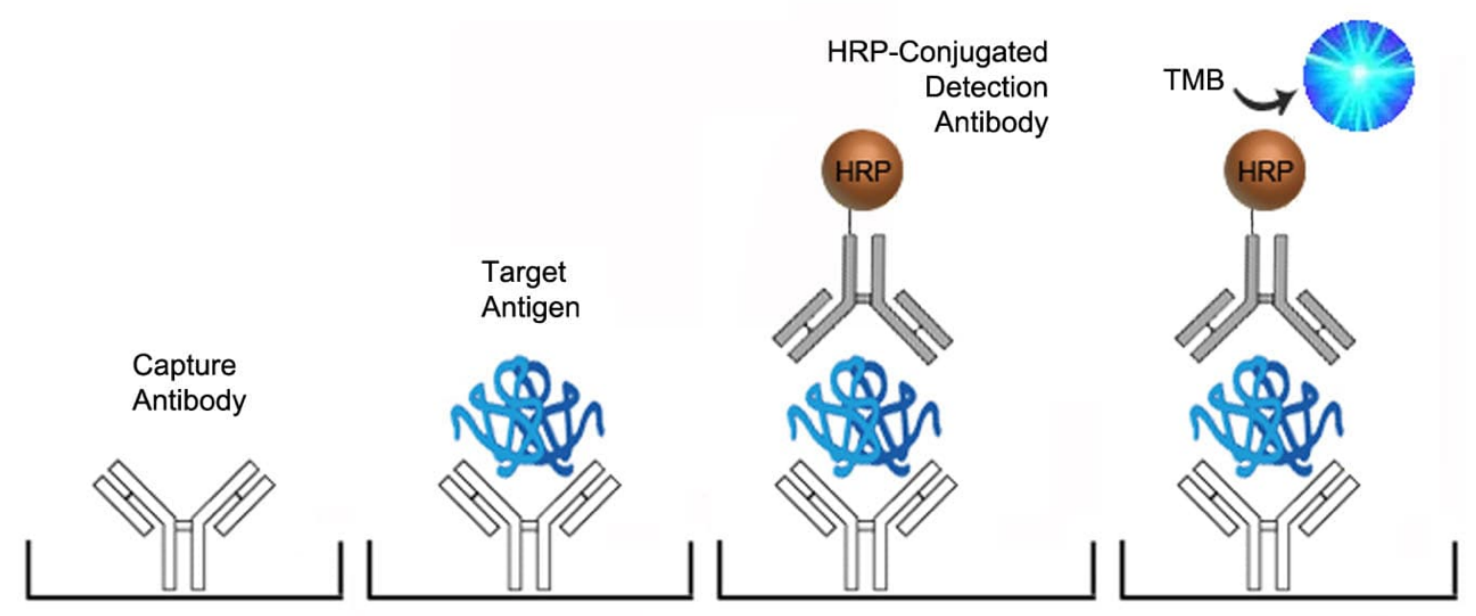

Figure 2: Schematic Representation of a Sandwich ELISA [38]

A sandwich ELISA involves a matched antibody pair consisting of a capture antibody and detection antibody. Both antibodies bind to the target analyte in different locations, sandwiching the analyte between the two. The first step in this type of ELISA is to coat the wells of the test plate with the capture antibody. The plate is then washed to remove any unbound capture antibody and a blocking agent is added to prevent non-specific binding [29]. Next, the sample is added to the wells and the capture antibodies bind to any target analyte present in the sample. The plate is then incubated to promote antibody/analyte binding, followed by a wash to remove any unbound analyte. Conjugated detection antibody is added next and binds to the target analyte, sandwiching it between the capture antibody and itself. Another wash is then performed to wash away any unbound detection antibody. Next, a detection agent, such 
as streptavidin-HRP, is added and bound to the conjugated detection antibody. After a final wash, a substrate solution like TMB is added to react with the detection agent and cause a color change. The intensity of this color change depends on the concentration of the analyte captured from the sample. The reaction is then stopped by adding an acidic solution, which turns the plate solutions a different color [29].

A competitive ELISA is often performed when only one antibody for the analyte is available or the analyte is so small that only one antibody is able to bind to it. In this type of ELISA the wells of the plate are first coated with the target analyte and a blocking agent is added to prevent non-specific binding. Next, the sample is added to the wells, followed by the detection antibody. If the target analyte is present in the sample, the detection antibody will bind to both the bound analyte in the wells as well as the analyte in the solution. Any detection antibody bound to the analyte in solution is then washed away in the wash step. Thus, once the detection agent and substrate are added to the wells, only the detection antibody that is bound to the analyte coated on the wells will generate a signal. A weaker signal suggests that a large number of detection antibody was washed away, indicating a large concentration of analyte in the sample solution. Therefore, a competitive ELISA determines how much analyte is present in a sample by observing the reduction of signal in the plate wells [29].

The main difference between a lateral flow assay and an ELISA is that an ELISA is able to give quantitative results. Instead of just having a color change indicate a yes/no response to whether or not target analyte is present in a sample, an ELISA can indicate the concentration of the target analyte based on the intensity of the color change. For this reason, the ELISA is becoming the preferred assay for diagnostic devices. Since many diseases can be marked by an increase or decrease in biomarkers normally present in the body, a quantitative test is needed to 
determine if there is a change in the biomarker concentration. For the purposes of this thesis, ELISA techniques will be integrated into the paper format of a lateral flow assay within a microfluidic chip. This allows the POC diagnostic device to display quantitative results while still conveniently utilizing a paper platform. For these ELISA techniques to be specialized for stroke diagnosis, specific biomarkers must be targeted.

\section{Stroke Biomarkers}

The use of biomarkers for the diagnosis of disease has become commonplace in the medical world. In search of a better diagnostic method, researchers have begun investigating potential biomarkers for stroke. The discovery of a biomarker that can predict the occurrence of a stroke will allow for a quicker diagnostic process that no longer relies on accurate interpretations of MRI or CT images. As mentioned earlier, current neuroimaging techniques are expensive and time consuming. Furthermore, CT scans are rather insensitive toward ischemia and both MRI and CT scans may be unable to detect abnormalities if the stroke area is too small. For these reasons, strokes are often misdiagnosed or not treated in a timely manner. To develop a more accessible and accurate diagnostic tool, researchers are turning toward biomarkers to shape the future of stroke diagnostics.

Over the past few years, many potential biomarkers for stroke have been explored. These biomarkers typically originate in the brain and end up in the bloodstream due to brain cell damage. During a stroke, the blood-brain barrier (BBB) is broken due to endothelial cell death, allowing contents from the cytoplasm of these cells to leak across the BBB [30]. These proteins migrate into the bloodstream where they can be detected in the plasma of the patient. Although most of the studied biomarkers originate in the brain, there have been some of nonbrain origin that still show a strong correlation with stroke. Across multiple studies, there are four 
biomarkers that seem to be the best predictors of stroke: S100 calcium binding protein B (S100b), matrix metalloproteinase-9 (MMP-9), N-methyl-D-aspartic acid (NMDA) receptor peptides, and glial fibrillary acidic protein (GFAP).

$\mathrm{S}-100 \mathrm{~b}$ is a calcium binding protein found in the cytoplasm of astroglial cells in the brain and spinal cord. This protein is able to cross the BBB when there is damage to these astroglial cells, which can occur during a stroke. The plasma concentration of S-100b is correlated with the extent of tissue damage observed in a stroke. The larger the infarct volume, the higher number of damaged astroglial cells and thus a higher concentration of S-100b that is able to leak from the brain into the bloodstream. However, a study conducted by Reynolds et al showed no significant differences in S-100b concentration levels within the first 6 hours of stroke symptom onset. This suggests that $\mathrm{S}-100 \mathrm{~b}$ alone is not a great biomarker for diagnosing stroke in a time efficient manner. However, it is still a good indicator of stroke and infarct volume size outside of the initial 6 hours. For this reason, S-100b is typically used in a panel with other biomarkers to aid in the diagnosis of stroke [31]. Since S-100b is unable to accurately predict stroke in the first 6 hours of onset, this biomarker is more helpful when diagnosing patients who have already passed their window for treatment with tPA.

Another common biomarker used for the diagnosis of stroke is matrix metalloproteinase9 (MMP-9). MMP-9 is a protein that is found in the cerebral tissue of the brain and is responsible for the degradation of extracellular matrix proteins. MMP-9 is released in the brain in response to injury and contributes to the pathological pathway of stroke by degrading extracellular proteins that are needed to maintain homeostasis. This leads to the breakdown of the BBB and allows for the release of MMP-9 and other proteins into the bloodstream. An increase in MMP-9 concentrations has been seen in both ischemic and hemorrhagic patients 
upon ER admittance, indicating an early release of the protein across the BBB [31]. This is desired in a stroke biomarker to diagnose the patient within the first 6 hours of stoke symptom onset. Furthermore, an increase in MMP-9 levels in patients who are treated with a thrombolytic therapy, such as tPA, has been shown to predict the development of intracerebral hemorrhage [32]. Due to this, MMP-9 can help predict if a patient will experience hemorrhagic complications after treatment with tPA and can thus be used to determine if alternative treatment is necessary.

NMDA receptors are found on neuronal cells in the brain and are shown to fragment into peptides in response to ischemic events. In a study conducted by Dambinova et al., the serum concentration of NMDA receptor peptides was significantly higher in ischemic stroke and transient ischemic attack (TIA) patients than in the controls. However, there was no change in NMDA peptide levels in intracranial hemorrhagic stroke patients [3]. For this reason, NMDA receptor peptides can serve as a biomarker for ischemic stroke, but not hemorrhagic stroke. This allows for this biomarker to differentiate between ischemic and hemorrhagic stroke, which is a crucial step in the stroke diagnostics process. Furthermore, studies have shown that the diagnosis of ischemic stroke or TIA using these peptides has a high sensitivity (97\%) and specificity (98\%) within the first 3 hours of symptom onset [3]. This is much desired for stroke diagnostics since patients with ischemic stroke can only be treated with tPA within the first 3 hours of symptom onset [6]. Since serum concentrations of NMDA receptor peptides show a significant increase shortly after symptom onset, this biomarker has the potential to shorten the diagnostic process for stroke and thus allow for a more time efficient treatment.

Glial fibrillary acidic protein (GFAP) has proven to be the most promising biomarker for differentiating between ischemic and hemorrhagic stroke. Since this protein is specific to the 
brain, there are less confounding variables in the diagnostic process since only brain related injuries can trigger its release. Furthermore, in a study conducted by Foerch et al. the window of opportunity for distinguishing between hemorrhagic and ischemic stroke using GFAP concentrations was shown to be $2-6$ hours after stroke symptom onset [3]. Since this window falls within the time constraints for tPA treatment, GFAP can help detect/distinguish between the two types of stroke in a timely manner so that the stroke may be treated quickly and efficiently. For this reason, GFAP is the primary biomarker that should be targeted by the microfluidic device developed in this thesis.

\section{Project Specific Biomarker Selection: GFAP}

For the microfluidic chip to serve as a diagnostic tool for stroke, the ELISA assay performed on the chip must target a single biomarker or panel of biomarkers. Ideally, a panel of biomarkers would be used since different markers are able to predict different aspects of a stroke and related complications. Furthermore, by targeting multiple biomarkers the device will provide the results of numerous tests all at once. In combination, these biomarkers will provide a more accurate prediction of the patient's condition. However, for the purposes of this thesis, only one biomarker will be targeted. Due to its ability to quickly distinguish between intracranial hemorrhagic and ischemic stroke, it was determined that the best biomarker to target is GFAP.

GFAP is able to reliably distinguish between intracranial hemorrhagic and ischemic stroke because of the different protein release times seen by the two. In multiple studies, it has been shown that GFAP has a delayed release in ischemic stroke patients, reaching maximum serum concentration levels between 2 to 4 days after symptom onset [33]. On the other hand, in a study conducted by Foerch et al., it was found that maximum GFAP concentration levels were 
reached within 2-6 hours after symptom onset in intracranial hemorrhagic stroke patients [3]. GFAP is likely released into the bloodstream faster during hemorrhagic strokes than ischemic due to the more sudden disruption of the blood-brain [33]. This difference in release time allows for a rapid distinction between the two types of strokes.

Ideally, the POC device would be used within the first 6 hours of symptom onset to allow for quick treatment of the patient. If high levels of GFAP are detected in the bloodstream within those first 6 hours, it can be predicted that the patient has suffered an intracranial hemorrhagic stroke. In the study conducted by Foerch et al., a cutoff point for the GFAP concentration level was found to be $2.9 \mathrm{ng} / \mathrm{L}$. Thus, any concentrations above $2.9 \mathrm{ng} / \mathrm{L}$ in the first 6 hours of symptom onset is considered to be high and likely indicates an intracranial hemorrhagic stroke has occurred. This particular study saw a 0.79 sensitivity and 0.98 specificity using this cutoff concentration for the differentiation of intracranial hemorrhagic stroke from ischemic stroke [33]. However, if the test is performed at a later time, it becomes less accurate at differentiating between the two types of stroke since GFAP levels will start to increase for both. Overall, GFAP is a strong indicator of intracranial hemorrhagic stroke within the first 6 hours of symptom onset and can thus be used as a rapid diagnostic marker. If tested for within the first few hours of symptom onset, this biomarker can help speed up the diagnostic process by providing a quick and easy way to differentiate between the two types of stroke. This will allow for the patient to be effectively treated in a more time efficient manner.

\section{Organization of the Remainder of the Report}

In chapter II, the methods taken to design and manufacture a 3D microfluidic chip utilizing both cellulose and nitrocellulose paper are described. Then, the protocol for successfully running an ELISA on the microfluidic chip will be explained in detail, highlighting 
several changes made throughout the testing process. In chapter III, the results of the ELISA experiments will be displayed. The implications of these results will be discussed in chapter IIII and the conclusions that can be drawn from them will be mentioned in chapter V. Lastly, in chapter VI, recommendations for future work will be discussed. 


\section{MATERIALS AND METHODS}

Throughout this project, many steps were taken to develop a functioning 3D microfluidic chip and its housing component. First, several 3D chips were developed throughout the process of determining an optimal chip design. The channel widths utilized in the chip designs were selected based on thesis work done by Jennifer Ward and Mackenzie Tageson. Housing components were designed and manufactured for each 3D chip to optimize pressure application to the chips. Dye tests were performed to verify connectivity and successful control of fluid flow throughout the 3D microfluidic chips. Development times were collected during these dye tests to determine an optimal pressure.

The second set of experiments performed in this project were aimed toward integrating ELISA techniques onto the 3D microfluidic chip. These experiments utilized the proteins and antibodies needed for an actual diagnostic device to function. First, the protein, antibody, buffer, and wash solutions were prepared to have specific concentrations for the ELISA test. Next, individual 2D chips were treated appropriately with these solutions. After being treated, these chips were reassembled in the housing and the ELISA experiment was performed. After several attempts, the protocol for the process described above was finalized. For this thesis to be considered a success, all of these experiments must be completed with satisfactory results.

\section{D Paper Microfluidic Chip Design and Fabrication}

Throughout the process of developing the 3D microfluidic chip, the chip design was changed several times due to the results of optimization tests and the acquisition of new knowledge. Each chip design was developed using AutoCAD. The initial chip design was meant to support a blotting assay type, where fluid primarily travels vertically with little lateral flow. To accommodate for this vertical flow, the channels were designed to be circular dots that 
overlapped layer to layer, allowing for fluid to flow between layers through these overlapping circles. This minimizes lateral flow by eliminating the need for long fluid-routing channels, which are typically used to connect layers. Based on the combined results from Jennifer Ward and Mackenzie Tageson's theses, the optimal channel widths appeared to be $1.6 \mathrm{~mm}$ to $2.5 \mathrm{~mm}$ [19,34]. For this chip, the detection layer and fluid-routing layer were designed to have circular channels with diameters of $2.5 \mathrm{~mm}$. Since the whole blood filter and conjugate layer contributed little to the fluid-routing path they were designed to have larger diameters to serve as a sample pad. The initial chip design can be seen in Figure 3 below.

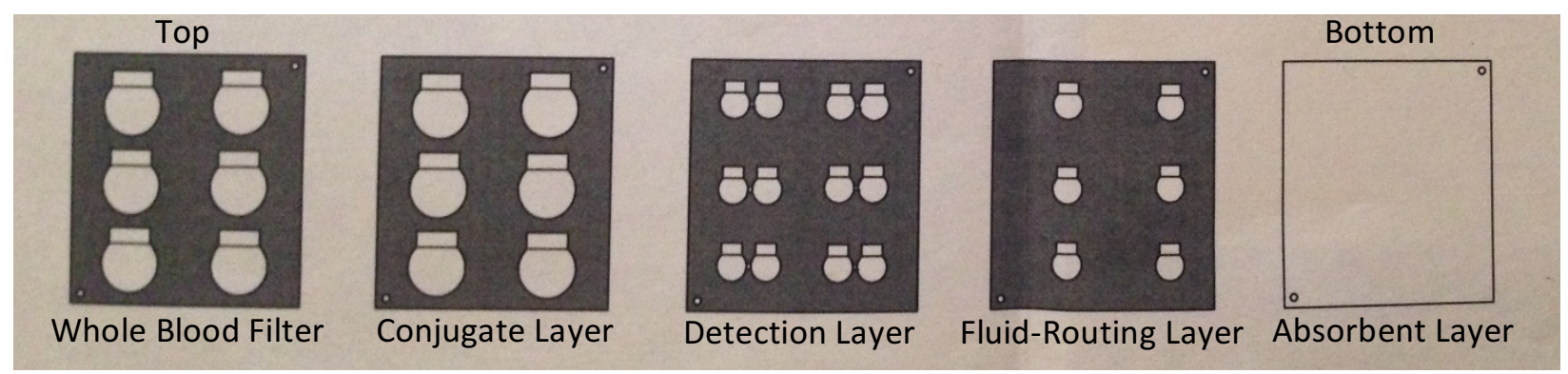

Figure 3: Initial 3D Microfluidic Chip Design

As seen in Figure 3, each circular region is topped with a rectangular area. These rectangular areas were incorporated into the design to indicate where pegs from the housing component would come into contact with the chip, creating pressure points atop each circular region. Six sample ports were included allowing for six tests to be run simultaneously. With the ability to run multiple tests at once, one channel could be designated as a control channel.

However, before this chip was manufactured, the design was discussed with both Dr. Clague and Dr. Howes, the thesis and biology advisors. Based on advice from Dr. Howes, it was decided that the design needed to include a larger channel area to accommodate for the multiple wash steps that take place during an ELISA. After much discussion, Dr. Clague suggested the use of a pin-wheel design that was able to spin, opening up new channels with each spin. Building off 
this pin-wheel idea, a circular chip was designed with adjacent channels that each sample port could be spun over, accommodating for multiple wash steps. This chip design can be seen in Figure 4 below.

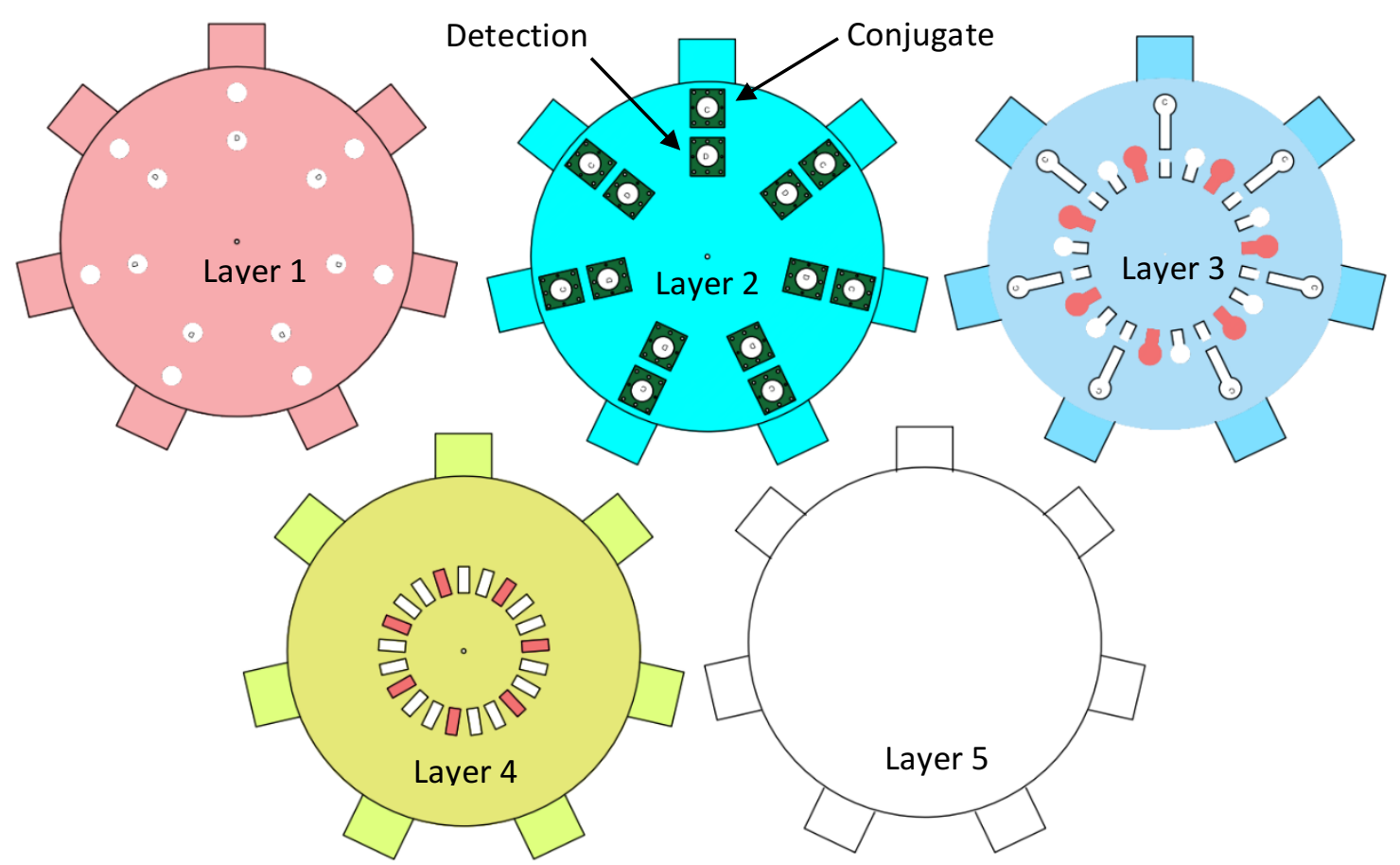

Figure 4: Second 3D Microfluidic Chip Design (First Circular Design)

As seen in Figure 4, the first layer of the chip acts as a sample pad, the second layer consists of the conjugate and detection regions, the third layer consists of the multiple fluidrouting channels available for each sample port, the fourth layer acts as a fluid-routing layer, and the fifth layer serves as the absorbent pad. Spokes were added around the outside of each layer to allow for an easy grip of the chip while it is in its housing. This was a necessary design feature since the top two layers of the chip needed to be spun while in the housing to direct fluid into new, dry channels on layer 3. The dimensions of the channels on this chip were again chosen based on the theses work of Mackenzie Tageson and Jennifer Ward. The details of these dimensions can be found in Appendix A. To create the hydrophobic barriers on each layer 
shown above (colored areas), a wax-printing technique was used. The wax was printed onto cellulose paper using the XEROX Phaser 8560 wax printer, shown in Figure 5.

The cellulose paper used was Whatman with a pore radius of 11 microns. The cellulose paper was fed into the upper tray of the printer to adjust for its smaller size. The designs were printed from an AutoCAD file and the print window size was adjusted to fit the cellulose paper.

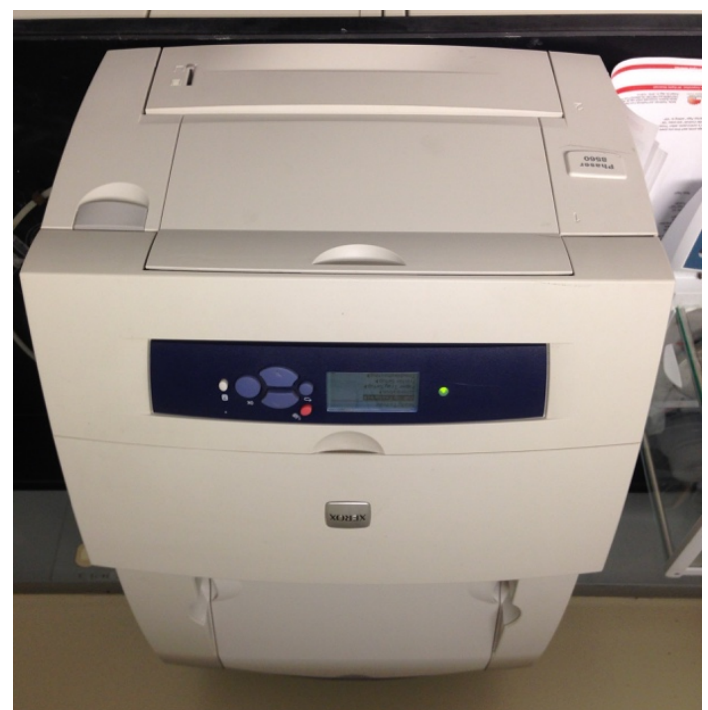

Figure 5: Xerox Phaser 8560 Wax Printer Used to Print the Microfluidic Chips

Once all five layers were printed out, they were individually placed onto a Clarkson digital, ceramic top hot plate. The hot plate was set at $125^{\circ} \mathrm{C}$ and each cellulose membrane was placed on the plate for about 60 seconds, or until the wax was melted all the way through the paper. To determine if the wax was melted all the way through, the cellulose paper was flipped and examined to see if any white spots still existed on wax-covered areas on the membrane. The membrane was heated on the hot plate until no white patches were seen on either side. Tweezers 
were used to push any lifted areas on the membrane down onto the hot plate to ensure successful melting. The hot plate that was used can be seen in Figure 6 .

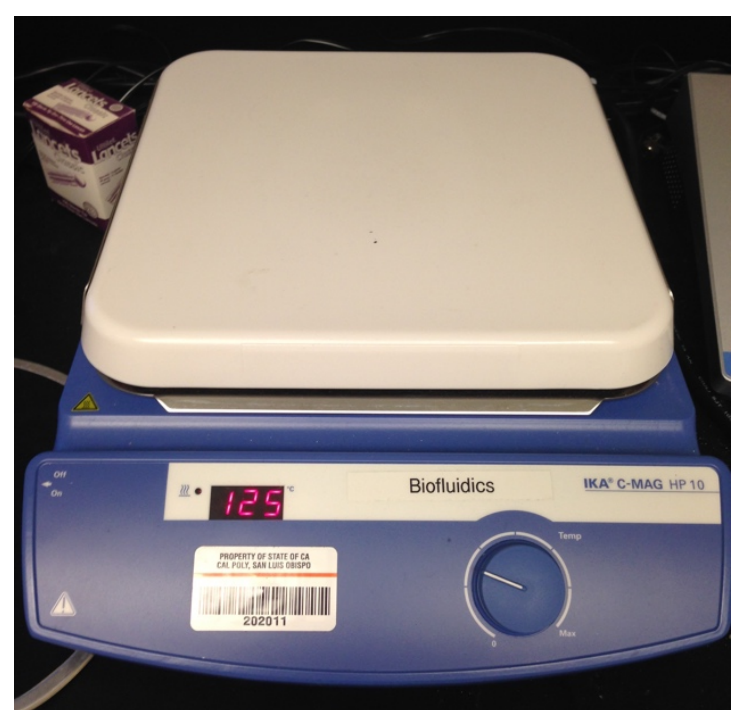

Figure 6: Clarkson Digital Hot Plate

Used to Melt the Wax on the Microfluidic Chips

After the wax was melted through each 2D chip, the chips were cut out appropriately.

Each chip was cut out along its outside edge and the center hole was cut out to allow for a screw to be pushed through it while in the housing. They were now ready to be assembled into a 3D chip and placed within the housing to perform dye testing. The dye testing procedures will be discussed later in this section. Based on the results of the dye testing performed on the first circular chip, a final chip was designed. This design can be seen in Figure 7. 


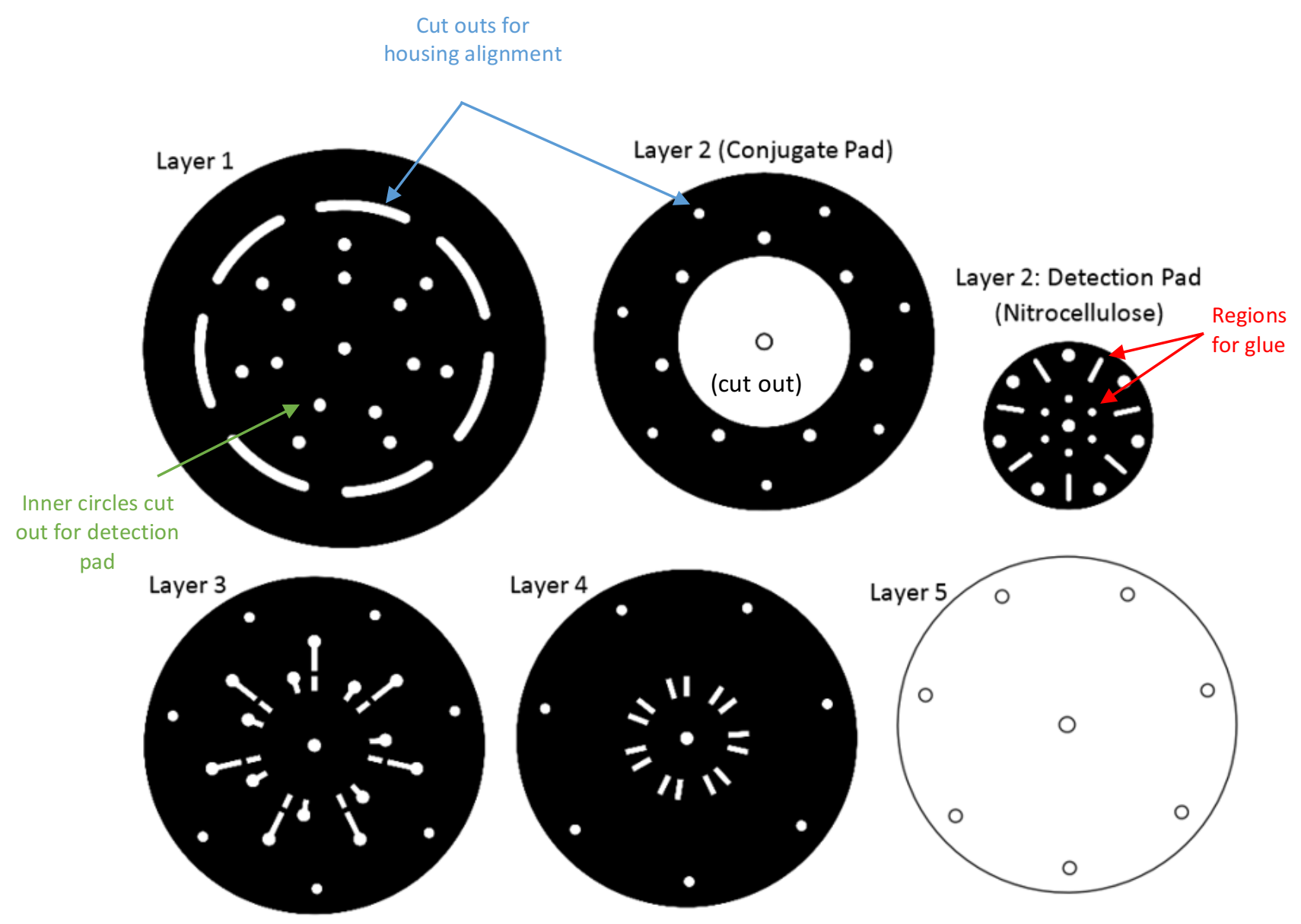

Figure 7: Final Chip Design

Based on results from dye testing with the first circular chip, the channel dimensions on the final chip were increased. These final dimensions can be found in Appendix A. This chip was manufactured using the same process as described for the first circular chip. When cutting out the layers, the seven outer circles on each layer (or elongated regions on layer 1) were also cut out to allow for the screws to go through the entire chip in these areas while in the housing. This helps keep the chips aligned in the housing. The elongated regions cut out on the first layer allows for this layer to be easily rotated in the housing without removing the screws. This enables the user to spin the top layer of the chip twice during the testing process, opening up new channels on layer 3 with each spin and thus accommodating for multiple washes. Furthermore, 
the top layer is slightly larger than the rest so that its outer edge extends out from the housing and allows for the user to grab and spin this layer without removing the chip from the housing. The detection pad has designated areas for glue (the spokes in between each detection region and the smaller circles toward the middle) which allow for the layer to be easily glued to the top layer of the chip during assembly. For the purposes of the dye testing, each layer was printed on a cellulose membrane. Once the layers were printed and the wax was melted through, the 2D chips were ready to be assembled and placed within the new housing.

\section{Housing Design and Fabrication}

To perform dye experiments on the printed 3D chips, housings were designed and fabricated for both chip designs. These housings are needed to apply pressure to the chips to ensure fluid connectivity between layers. Without pressure, there may be gaps of air between the chip layers causing a disruption to fluid flow since it cannot travel by capillary action across these gaps. As seen in Figure 4, the first circular chip design has several circular regions on the top layer of the chip. The outer ring of circles is where the samples are first introduced onto the chip. The inner ring of circles is where the detection region is located and where the second wash is introduced. To allow for the introduction of the sample and washes, the housing must have openings at these specific regions. AutoCAD was used to design the housing components. The AutoCAD design of the top and bottom components of the housing for the first circular chip can be seen in Figure 8 . 

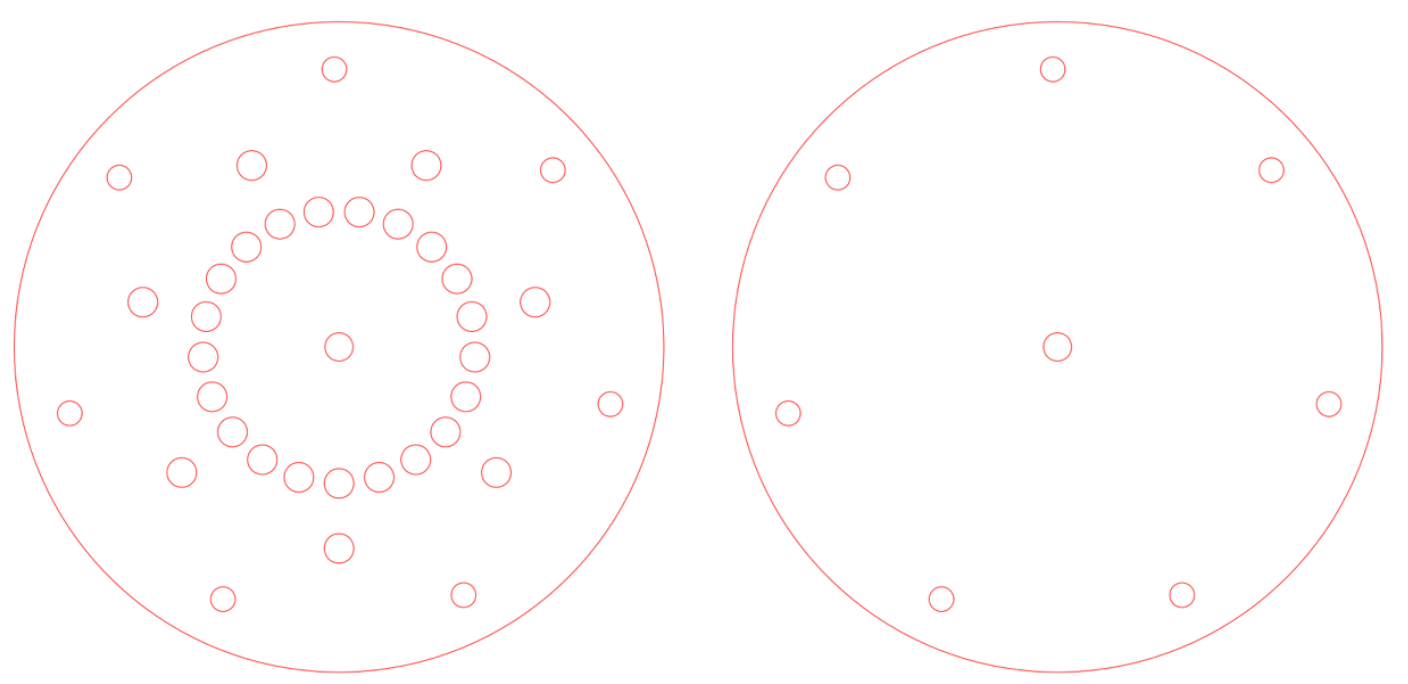

Figure 8: Top (left) and Bottom (right) Components of First Housing

The details of the dimensions for this housing can be found in Appendix A. Once this design was finalized, the housing was fabricated using a laser cutter in the IME lab on Cal Poly's campus. A twelve-inch squared piece of acrylic with a thickness of 0.060 inches was placed in the laser cutter. The AutoCAD file was then opened on the laptop connected to the laser cutter and the design was cut out of the acrylic sheet. After the cutting was complete, the housing components were separated from the excess acrylic pieces and brushed off. Larger holes were strategically located in the middle and around the outside of the housing to allow for the insertion of screws. The screws used have a $1 \frac{1}{2}$ inch thread length with a $1 / 4$ inch diameter. The two housing components were held together by screwing the screws into washers located beneath the bottom piece. When screwed together, the acrylic housing applies pressure to the chip by sandwiching the chip in between the two pieces. The housing for the first circular chip can be seen in Figure 9. 


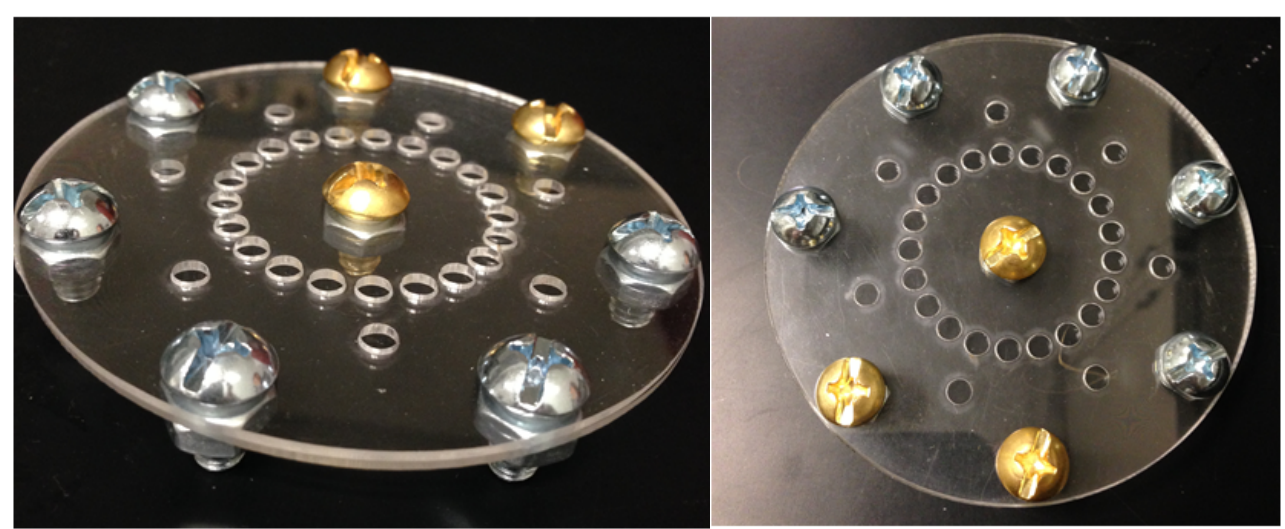

Figure 9: Assembled Housing for the First Circular Chip

Based on the results from dye testing with the first chip and housing pair, a final chip was designed and thus a final housing was made. Despite the differences between the first circular chip and final chip, the housings were both made from the same design. However, due to the increase in channel size and overall chip size, the initial housing design was modified to accommodate for these changes. Using the AutoCAD design of the first housing seen in Figure 8 , the size of the circular cut-outs and the entire housing component itself were increased. The dimensions of the final housing can be found in Appendix A. The laser cutter was then used to cut this modified design out of acrylic sheets. To increase the pressure applied by the housing, a thicker acrylic with a 0.50 inch thickness was used for the bottom component. Acrylic with a 0.060 inch thickness was still used for the top component. After both components were cut out, a drill press was used to make the screw holes in the bottom housing piece a little larger. This was done to fit the 0.266 inch diameter hex threaded inserts needed for screw insertion. The hex 
threaded inserts were inserted into the bottom housing piece using a benchtop press similar to the one seen in Figure 10.

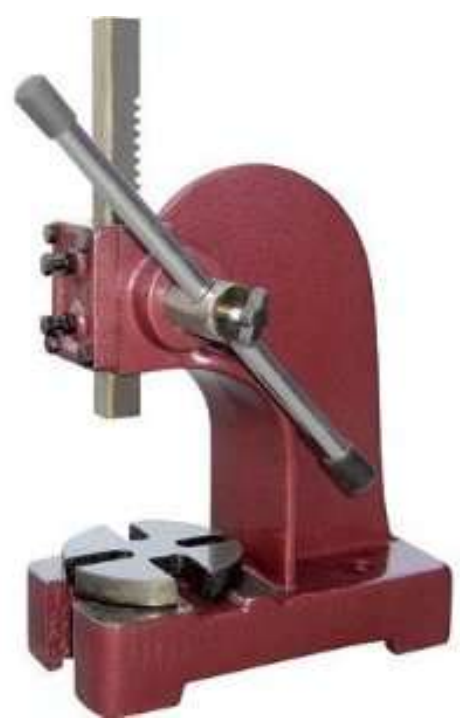

Figure 10: Benchtop Press Used to Press the Hex Threaded Inserts into the Housing

Once the hex threaded inserts were placed within the bottom housing piece, screws with knurled heads were screwed into the inserts, holding the top and bottom housing components together. These screws were chosen because they make it easier for the user to tighten and loosen the housing with their bare hands. The screws had 8-32 threads with a 3/8 inch length. Since the chip was expected to be spun in the housing for each wash step, a shoulder screw was used in the middle to allow for a smoother spin. The assembled final housing can be seen in Figure 11. 


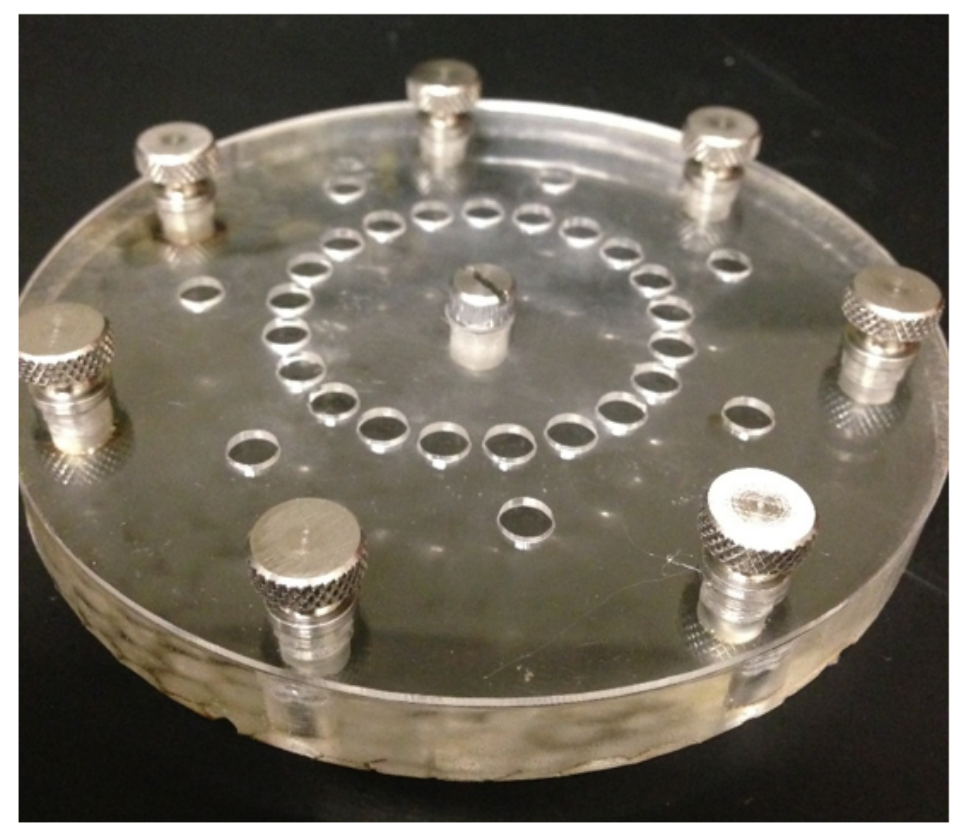

Figure 11: Assembled Final Housing

\section{Dye Testing}

\section{Fluid Connectivity}

Once the first circular chip and housing were fabricated, dye experiments were performed to ensure fluid connectivity throughout the chip. First, the chip was assembled according to Figure 4, starting with layer 5 on bottom and stacking each consecutive chip on top of the next, ending with layer 1 on top. During this process, it is crucial that the chips are carefully assembled so that the channels line up perfectly from layer to layer. Next, the assembled chip was then positioned within the housing. Each screw was tightened with a screwdriver until it reached a point where it was tight enough to give resistance. This ensured the housing was screwed with a uniform tightness at each screw, but was not tight enough to collapse the pores of the chip membrane. The assembled microfluidic device can be seen in Figure 12. 


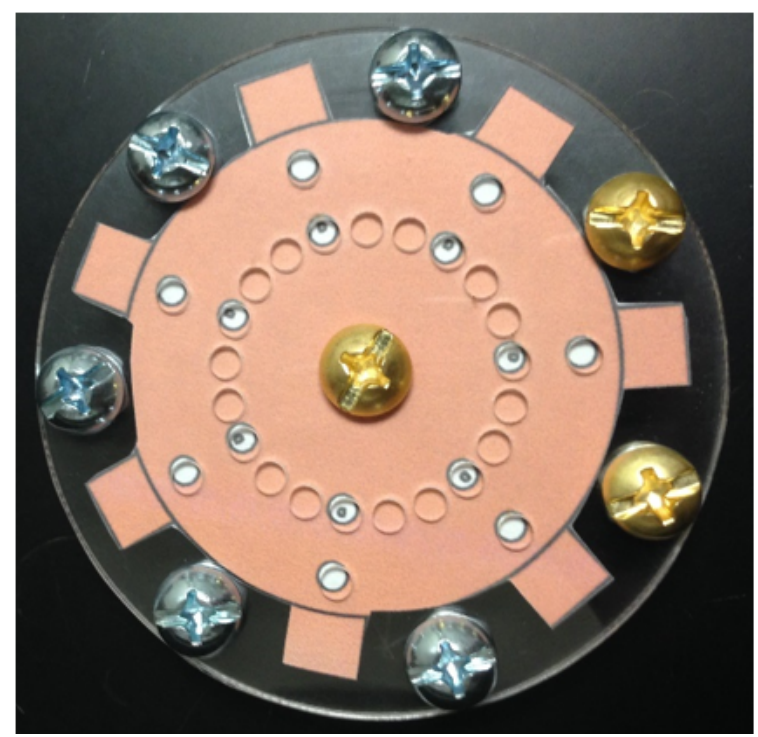

Figure 12: First Microfluidic Device Assembled with Screws

Once the first circular chip was assembled in the housing, it was ready for dye testing. The dye solution was created by mixing one drop of blue food coloring into $20 \mathrm{ml}$ of DI water. A $20 \mu 1$ pipette was then used to pipette $20 \mu \mathrm{l}$ of the dye solution onto the sample pad for each channel on the chip (the outer ring of circles seen in Figure 12). Once the fluid was fully wicked through the chip, the chip was removed from the housing and examined to see if the fluid remained in the designated channels.

Based on the initial dye testing results, it was determined that the screws were not providing enough pressure to the chip. For the next set of dye testing, paper clamps were used to apply pressure along the outside of the housing to determine if the first circular chip was able to successfully route fluid under more pressure. The clamped device can be seen in Figure 13. 


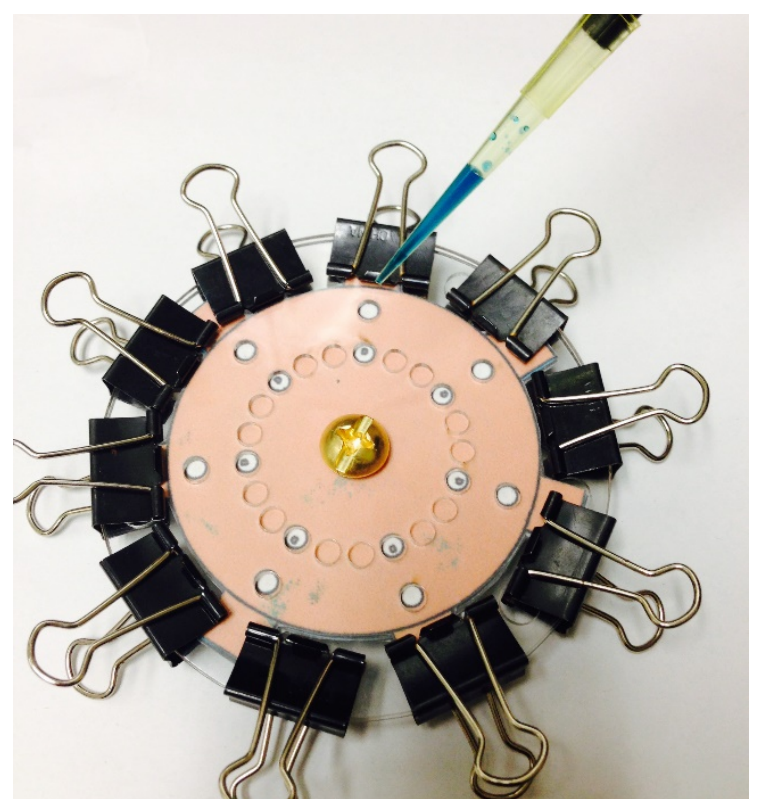

Figure 13: First Microfluidic Device Assembled with Paper Clamps

Once the device was clamped appropriately, the dye experiments were performed again using the same procedure as described above. As can be seen in Figure 13, $20 \mu 1$ of blue dye solution was pipetted onto the chip at each of the seven circular regions in the outer ring of the chip. Once the fluid was completely wicked through the chip, the chip was removed from the housing and examined once again.

Based on the results from dye testing with the first microfluidic device, a new chip and housing was created. The chip was assembled according to Figure 7, starting with layer 5 on bottom and stacking each consecutive layer on top of the next, ending with layer 1 on top. During this process, it is crucial that the chips are carefully assembled so that the channels line up perfectly from layer to layer. The assembled chip was then positioned in the housing to create the final microfluidic device. Each screw was tightened by hand as far as it would go to tighten the housing as much as possible. Since turning the screws by hand has a lot less power than using a screwdriver, collapsing the membrane pores was not a concern. Once the device was assembled, dye testing was performed using the same process as described above, except with 
red dye instead of blue. A pipette was used to pipette $20 \mu 1$ of the red dye solution onto each sample pad region (the outer circles). The final microfluidic device can be seen in Figure 14, with the pipette over the region where the dye solution was introduced for each channel.

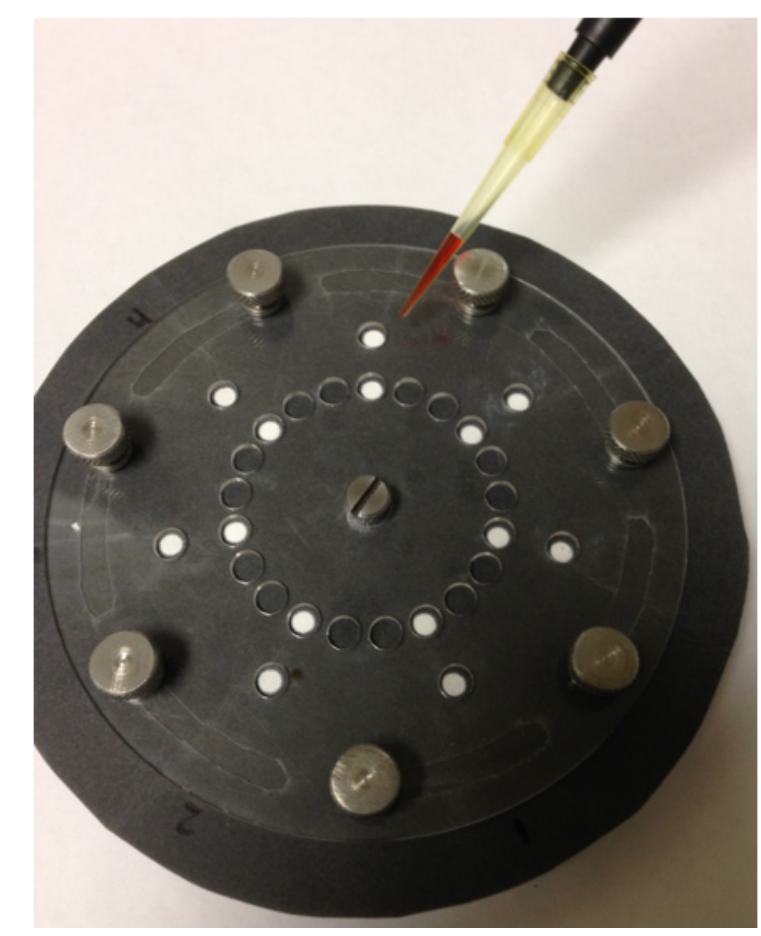

Figure 14: Final Microfluidic Device Pictured during Dye Testing

Once the fluid was completely wicked through the chip, the chip was removed from the housing and examined to see if the fluid remained in the designated channels.

\section{Conjugate Pad}

Once fluid connectivity was confirmed in the final microfluidic device, dye testing involving the conjugate pad was performed. In the first dye experiment, the conjugate pad was made out of cellulose paper. In later tests involving real reagents, the detection antibody will be dried onto this conjugate layer in the 3D chip. Once the sample flows through the conjugate pad, the detection antibody is expected to resolubilize and continue flowing through the remainder of the chip. To simulate this drying and resolubilizing process, red dye was dried onto the 
conjugate pad made from cellulose paper. The dyed cellulose membrane was left to dry over night at room temperature. This conjugate pad can be seen in Figure 15.

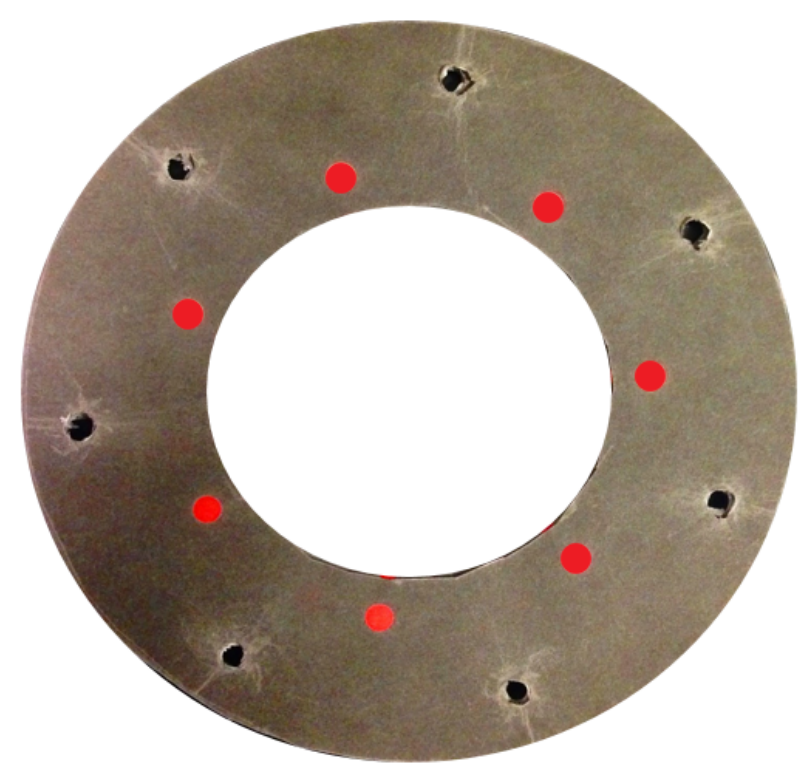

Figure 15: Dyed Conjugate Pad Made from Cellulose Paper

Once the red dye was dried onto the conjugate pad, the layer was integrated into the 3D chip appropriately beneath the sample pad layer. The chip was assembled in the same manner as described in the initial dye experiments and was then positioned in the housing. The screws were tightened by hand as far as they could turn. A $20 \mu$ pipette was then used to pipette $20 \mu 1$ of plain DI water onto each sample pad region. The DI water was expected to flow through the conjugate pad, resolubilizing the red dye and thus spreading the red dye throughout the remainder of the chip. The results of this experiment will be discussed in the Results section of the report.

In the second experiment involving the conjugate pad, the pad was made from a fiberglass membrane. The membrane used was Millipore $10 \mathrm{~mm}$ by $300 \mathrm{~mm}$ fiberglass strips. The conjugate pad was cut from a strip into a small rectangular piece that fit over the sample pad region of the chip. Drops of red food dye were then added to the fiberglass conjugate pad until 
the entire pad was saturated in the dye. The pad was then left to dry over night at room temperature. Once the conjugate pad was dry, it was attached on the bottom side of the top layer of the chip, covering the sample pad area. The pad was attached to the top layer using super glue on all four corners of the pad. The top layer of the chip with the conjugate pad attached can be seen in Figure 16.

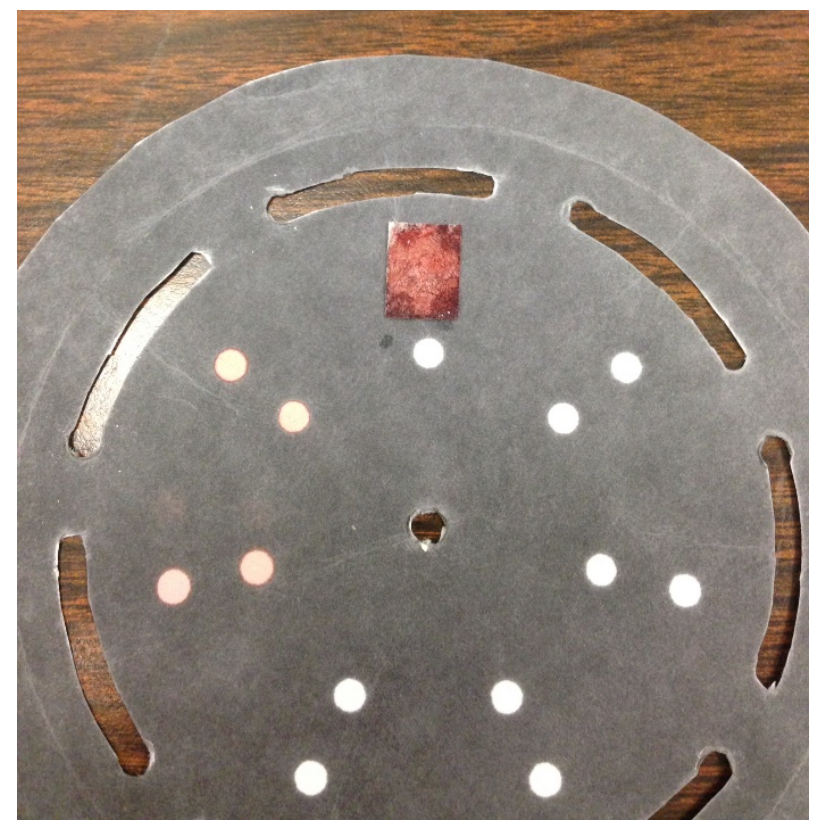

Figure 16: Dyed Fiberglass Conjugate Pad Attached to Top Layer of Chip

Once the conjugate pad was glued onto the bottom side of the lop layer of the chip, the entire chip was assembled in the same manner as described above and positioned in the housing. A $20 \mu \mathrm{l}$ pipette was then used to pipette $20 \mu \mathrm{l}$ of plain DI water onto the sample pad of the chip in the channel containing the attached conjugate pad. Once the DI water was wicked through the entire channel, the chip was removed from the housing to see if the dried dye was successfully resolubilized and spread throughout the remainder of the chip in the designated channel.

\section{Nitrocellulose Detection Layer}

Once fluid connectivity was confirmed for a fully assembled chip made out of cellulose paper, the detection layer was finally printed on a nitrocellulose membrane for the last set of dye 
experiments. Due to the cost and fragility of nitrocellulose paper, the previous experiments used a cellulose membrane for the detection layer. This was done to confirm fluid connectivity and finalize the chip design before any nitrocellulose paper was used. However, to prepare the final chip for testing with real reagents, the detection layer needed to be printed on nitrocellulose paper to allow for protein binding.

Since the nitrocellulose is a fragile membrane, it must be handled with great care during the printing process. First, the detection layer was printed out onto a regular sheet of paper. This was done to determine the correct size and paper orientation needed to feed the nitrocellulose membrane through the printer. The nitrocellulose was cut out and placed directly over the printed detection layer on the regular sheet of paper. All four edges of the nitrocellulose cut-out were then completely taped down onto the regular sheet of paper, with no free edges exposed. This paper setup can be seen in Figure 17. 


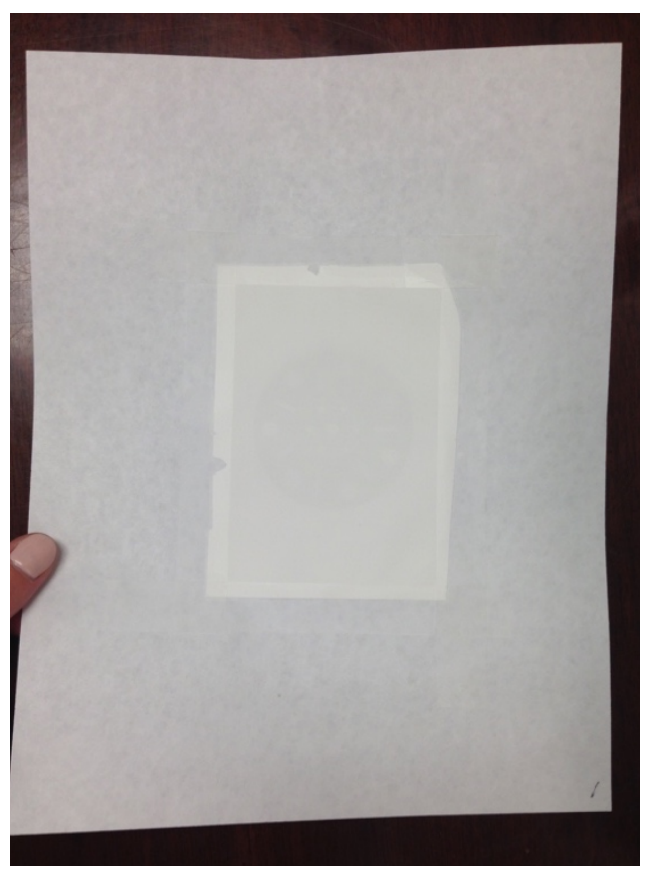

Figure 17: Paper Setup for Nitrocellulose Printing

The entire sheet of paper was then placed in the printer tray appropriately to ensure that the detection layer would print exactly onto the nitrocellulose region. Once the detection layer was printed onto the nitrocellulose paper, the nitrocellulose square was cut out from the taped edges. This process can be seen in Figure 18.

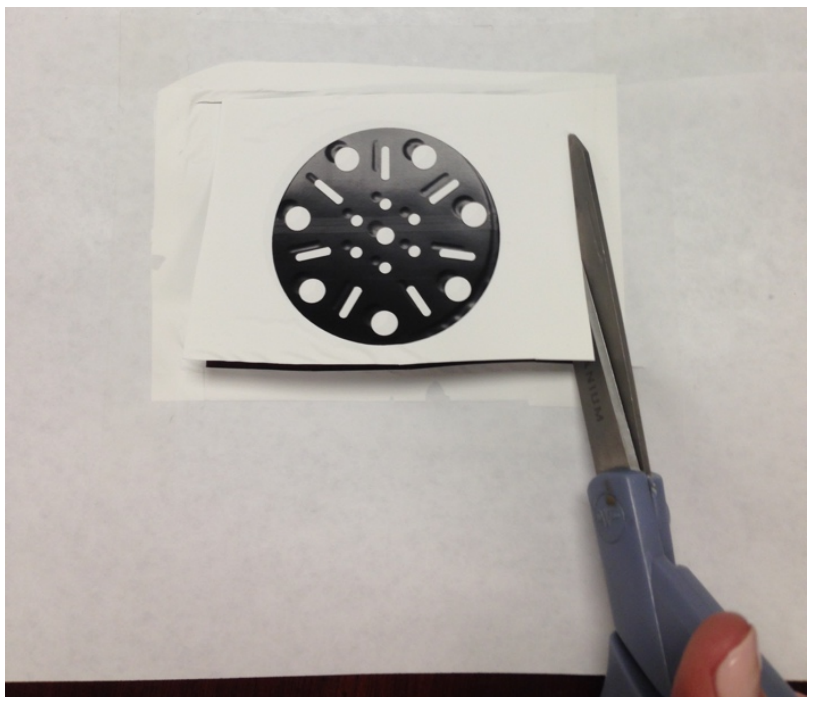

Figure 18: Cutting Strategy Used to Remove Nitrocellulose Layer from the Sheet of Printer Paper 
The nitrocellulose membrane, still backed by the regular paper, was then placed on the hot plate. The plate was heated to $125^{\circ} \mathrm{C}$ and the chip was left on it for about 60 seconds. Once the wax was melted through the nitrocellulose paper, the membrane become strong enough to peel away from the regular paper and was thus separated from it. This nitrocellulose paper was then placed back onto the hot plate until there were no white spots in the wax printed areas, ensuring that the wax melted all the way through. The detection layer was then cut out along the edges of its circular border. The layer was now ready to be integrated into the 3D chip.

To incorporate the detection layer into the 3D chip, the nitrocellulose membrane was attached to the bottom side of the top layer of the chip. Due to the messy nature of super glue, tape was taped over the designated areas for glue on the detection layer and the chip was taped to the top layer instead of glued. During this process, it was crucial to line up the circular channels on both the top layer and the detection layer. The top layer of the chip with the nitrocellulose layer taped to it can be seen in Figure 19. 


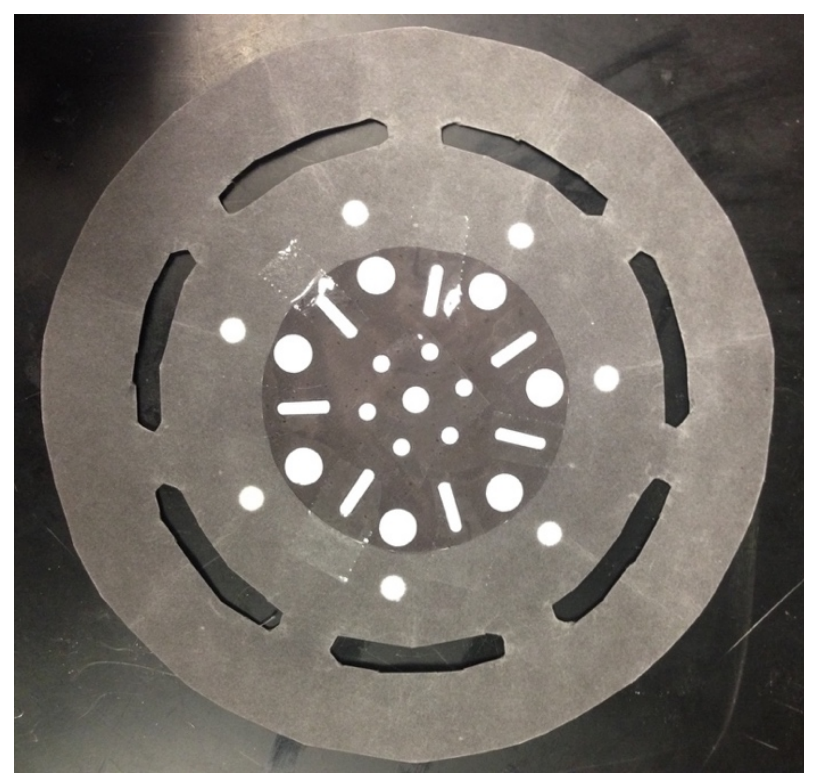

Figure 19: Nitrocellulose Detection Layer Taped to the Top Layer of the 3D Chip

Once the detection layer was successfully attached to the top layer of the chip, the entire chip was assembled in the same manner as described in previous dye experiments. The assembled chip was then positioned in the housing and the screws were tightened by hand as far as they could turn. Red dye solution was then made by mixing one drop of red food dye into 20 $\mathrm{ml}$ of DI water. Using a $20 \mu \mathrm{l}$ pipette, $20 \mu \mathrm{l}$ of the dye solution was pipetted onto each of the seven sample pad regions on the chip (outer ring of circles). Once the fluid was wicked completely through the chip, the chip was removed from the housing and examined to see if the fluid remained in the designated channels. Furthermore, the detection layer was observed to verify fluid connectivity through the nitrocellulose membrane.

\section{Development Time Testing}

After successfully completing the dye experiments, testing was performed to determine the development times of the fully assembled chip. A development time is the time it takes the fluid to travel from one point on the chip to the next. Three different development times were recorded: the time to the detection region, the time to the absorbent pad, and the time to the 
absorbent pad for the second wash. For the time to the detection region, the clock was started the moment the fluid was first introduced to the chip and stopped when the fluid reached the detection region. For the time to the absorbent pad, the clock was started the moment the fluid was first introduced to the chip and stopped when the fluid reached the absorbent pad. Lastly, for the time to the absorbent pad for the second wash, the clock was started the moment the wash fluid was introduced to the detection region and stopped when the fluid reached the absorbent pad.

To easily see where the fluid was on the chip, a red dye solution was used for this experiment. The solution was prepared by adding one drop of red food dye to $20 \mathrm{ml}$ of DI water. A full 3D chip, with both cellulose and nitrocellulose membranes, was fabricated and assembled appropriately in the housing. A $20 \mu 1$ pipette was then used to pipette $20 \mu 1$ of the dye solution onto one of the sample pads on the chip. The moment the fluid touched the sample pad, an iPhone 5 stopwatch was started. The chip was then observed closely to watch for the moment the red dye solution reached the detection region. As soon as the faintest bit of red fluid was seen on the detection region, the Lap button was hit on the iPhone timer to record the first development time. The chip was then observed again to watch for when the red fluid hit the absorbent pad; this was seen by holding the device up in the air and viewing the absorbent pad through the bottom housing piece. Once the red fluid was seen on the absorbent pad, the timer was stopped. The two times were then recorded. For the second wash step, the screws were loosened and the top layer of the chip was spun over the second channel on the layer beneath it. The screws were tightened once the channels were lined up correctly between the different layers. Lastly, $20 \mu \mathrm{l}$ of red dye solution was pipetted directly onto the detection region and the timer was started. The device was held up again to watch through the bottom housing piece for 
when the red dye hit the absorbent pad. Once the faintest amount of red was seen on the absorbent pad, the timer was stopped and the last development time was recorded. This process was repeated six more times for the remaining channels on the chip.

\section{Antibody Experiments: 3D Paper Microfluidic Device}

\section{$\underline{\text { Initial ELISA Test }}$}

To test the ability of the 3D paper microfluidic device to run an ELISA, an initial protocol was drafted referencing the theses work of Makenzie Tageson and Jennifer Ward for several procedural steps. However, research was also done on the specific antibodies and protein (troponin) used in this project to customize the ELISA protocol for these particular reagents. The calculations performed to come up with the following procedures can be found in Appendix B. First, the buffering and blocking solutions needed for the ELISA experiment were prepared. A BSA stock solution was created using the following procedure:

- Measure out $80 \mu \mathrm{l}$ of the $1 \mathrm{mg} / \mathrm{ml}$ stock solution of BSA

- Add $720 \mu \mathrm{l}$ of DI water to the solution

- This combination produces a $100 \mu \mathrm{g} / \mathrm{ml}$ BSA solution

A buffer solution using BSA was created using the following procedure:

- Measure out $24 \mu \mathrm{l}$ of the $1 \mathrm{mg} / \mathrm{ml}$ stock solution of BSA

- Add $216 \mu 1$ of DI water to the solution

- Dissolve $0.1 \mathrm{~g}$ of sucrose in $500 \mu \mathrm{l}$ of DI water and add $2 \mu \mathrm{l}$ of this solution to the BSA solution

- This combination produces a $110 \mu \mathrm{g} / \mathrm{ml}$ BSA buffer solution After these solutions were prepared, each chip layer was treated appropriately before being assembled into a 3D chip. 


\section{Detection Layer}

To prepare the nitrocellulose detection layer, the capture antibody needed to be dried onto the layer. To strengthen the binding of the capture antibody to the nitrocellulose region, the layer was coated in streptavidin and the capture antibody (anti-cardiac troponin T antibody) was biotinylated. The following procedure was used to biotinylate the capture antibody:

- Place the capture antibody vial in the Beckman Coulter Microfuge 16 for 10 seconds

- Measure out $50 \mu \mathrm{l}$ of the $2 \mathrm{mg} / \mathrm{ml}$ solution of the capture antibody (100 $\mu \mathrm{g}$ total)

- Centrifuge the Biotin Modifier vial for 10 seconds and add $5 \mu$ of it to the capture antibody solution

- Mix gently, then pipet onto lyophilized material

- Incubate at room temperature for 15 minutes

- Centrifuge the Biotin Quencher vial for 10 seconds and then add $5 \mu$ of it to the capture antibody solution

- Incubate at room temperature for 4 minutes

For the purposes of this experiment, two detection layers were prepared. Both 2D chips were first coated in streptavidin. To create the streptavidin solution, $36 \mu l$ of a $0.5 \mathrm{mg} / \mathrm{ml} \mathrm{stock}$ solution of streptavidin was mixed with $240 \mu \mathrm{l}$ of DI water. Then, $23 \mu 1$ of this solution was pipetted onto the six detection regions on each nitrocellulose chip (one region per chip was left as a control). Both chips were sitting on top of a piece of foil. The layers were dried in a humidity chamber for 10 minutes and then an additional 10 minutes in a chamber with a vacuum pump on. The humidity chamber with the vacuum pump attached can be seen in Figure 20 . 


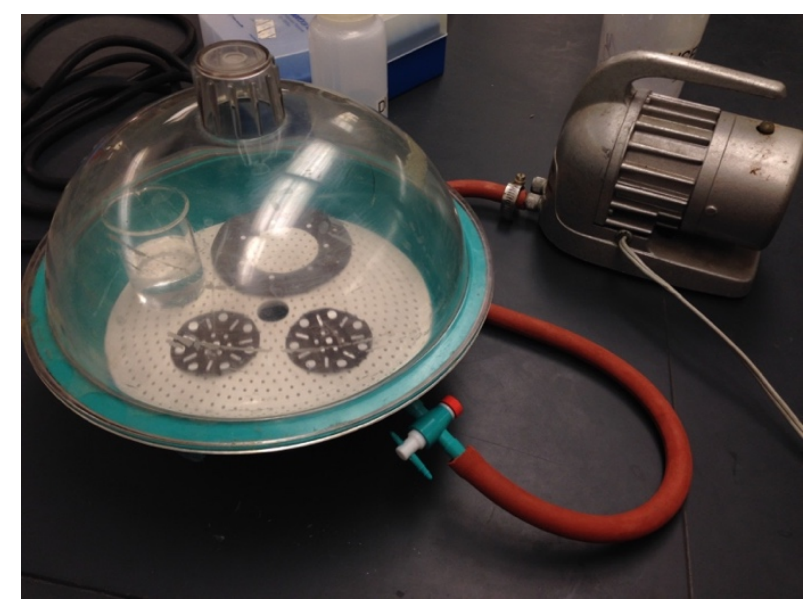

Figure 20: Humidity Chamber Attached to Vacuum Pump

Next, the capture antibody solution was created using the following procedure:

- Measure out $2 \mu \mathrm{l}$ of the biotinylated $2 \mathrm{mg} / \mathrm{ml}$ stock solution of the capture antibody

- Add $30 \mu \mathrm{l}$ of DI water to the solution

- This combination produces a $0.125 \mu \mathrm{g} / \mu \mathrm{l}$ solution of the capture antibody

The capture antibody solution was then ready to pipette onto the nitrocellulose chips. Since the area of each detection region is roughly half the area of a typical ELISA well, the typical amount of reagents used in an ELISA were halved for this experiment. Based on the pamphlets provided in the troponin/antibody pair kit, the typical amount of capture antibody used in an ELISA is $1 \mu \mathrm{g}$. Thus, for this test, $0.5 \mu \mathrm{g}$ of capture antibody was used on each detection region. To get this concentration, $4 \mu \mathrm{l}$ of the capture antibody solution was pipetted onto each detection region (six per chip). Both chips were then dried in a chamber with the vacuum pump on until visibly dry. 
To block the detection layer, $50 \mu \mathrm{l}$ of the BSA solution was pipetted onto each detection region on both chips. The chips sat on top of a piece of foil and the BSA solution was allowed to soak on the chip surfaces for 30 minutes. The BSA sitting on top of the nitrocellulose chip can be seen in Figure 21.

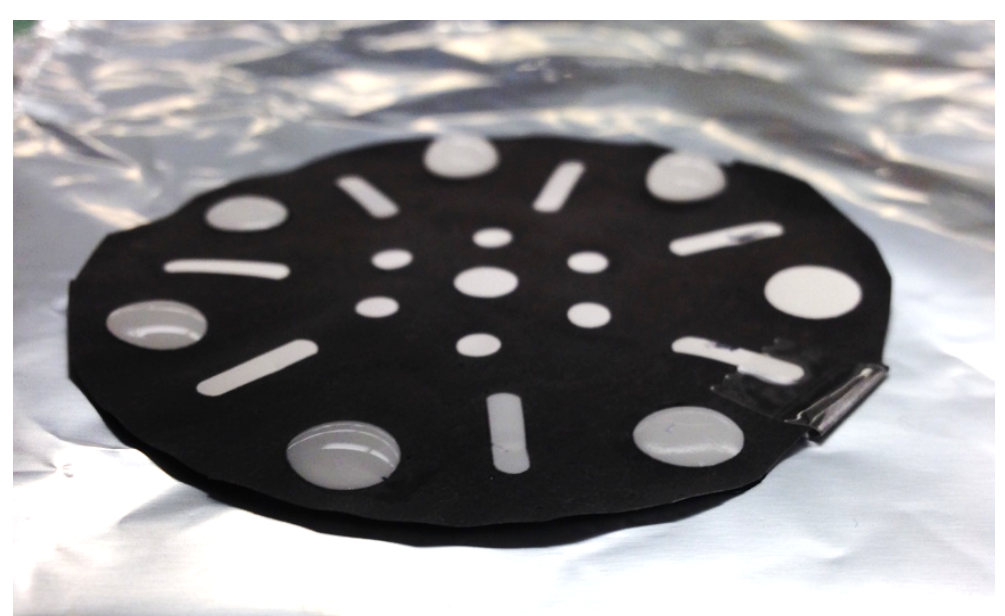

Figure 21: BSA Soaking on the Nitrocellulose Chip During the Blocking Step in the ELISA Procedure

Next, the membranes were washed with a $0.05 \%$ PBST stock solution by pipetting $50 \mu 1$ of the PBST onto each detection region for 15 minutes. This washing process was repeated two more times. Lastly, the chips were dried in a humidity chamber under vacuum until visibly dry. The detection layers were now ready to be assembled in the 3D chip.

\section{Conjugate Pad}

Due to previous complications with the fiberglass conjugate pad, it was decided that a cellulose membrane would be used for the conjugate pad. To prepare the conjugate pads, HRPconjugated detection antibody (HRP-conjugated anti-cardiac troponin T antibody) needed to be stored on the pad. First, a solution of the HRP-conjugated detection antibody was created using the following procedure:

- Measure out $5 \mu \mathrm{l}$ of the $0.9 \mathrm{mg} / \mathrm{ml}$ stock solution of detection antibody 
- Add $85 \mu 1$ of DI water to the solution

- This combination produces a $50 \mathrm{ng} / \mu \mathrm{l}$ solution of the detection antibody

According to the pamphlets that came with the troponin/antibody pair kit, the typical mass of detection antibody used in an ELISA well is $200 \mathrm{ng}$. Thus, $100 \mathrm{ng}$ will be used per conjugate region in this experiment since the region is about half the area of a typical ELISA well. To get this $100 \mathrm{ng}$ per conjugate region concentration, the following procedure was used:

- Measure out $24 \mu \mathrm{l}$ of the $50 \mathrm{ng} / \mu \mathrm{l}$ detection antibody solution

- Mix this $24 \mu$ l of detection antibody with the prepared buffer solution described above

- Pipette $22 \mu 1$ of this solution onto each region on both conjugate pads

- Dry the conjugate pads in a humidity chamber under vacuum until visibly dry The conjugate pads were now ready to be assembled in the 3D microfluidic chip.

\section{Sandwich ELISA}

Once both the detection layers and conjugate pads were prepared, the 3D chips were assembled appropriately. Since there was only one housing, the ELISA was run on each chip one at a time. This experiment is aimed toward detecting physiologically relevant levels of troponin in the bloodstream. In stroke patients, this concentration is greater than $50 \mathrm{ng} / \mathrm{L}$ [35]. Assuming that a typical blood sample is around $10 \mathrm{ml}$, the amount of troponin used should be greater than $0.5 \mathrm{ng}$ since this is the level that should be seen in a stroke patient. For this test, three different masses are used to see a ladder effect in the signal. The masses used are $1 \mathrm{ng}, 10$ ng, and 100ng. Since each chip contains seven channels, each mass was used on two channels (6 total) and the last channel was used as a negative control. The different troponin solutions were prepared using the following procedures: 
100 ng Sample

- Measure $2 \mu \mathrm{l}$ of the $1.93 \mathrm{mg} / \mathrm{ml}$ stock solution of troponin

- Add $77 \mu$ of DI water to the solution

10 ng Sample

- Measure out $2 \mu \mathrm{l}$ of the $100 \mathrm{ng}$ sample solution

- Add $18 \mu$ l of DI water to the solution

1 ng Sample

- Measure out $2 \mu \mathrm{l}$ of the $100 \mathrm{ng}$ sample solution

- Add $198 \mu \mathrm{l}$ of DI water to the solution

Once the troponin solutions were created, the ELISA was run on the 3D paper

microfluidic device. First, $4 \mu \mathrm{l}$ of the $1 \mathrm{ng}$ sample solution was mixed with $40 \mu \mathrm{l}$ of sterile PBS.

With the first two channels on the chip designated for the $1 \mathrm{ng}$ samples, $22 \mu \mathrm{l}$ of this mixture was pipetted onto each of those channels. Next, $4 \mu \mathrm{l}$ of the $10 \mathrm{ng}$ sample solution was mixed with 40 $\mu l$ of sterile PBS, and $22 \mu \mathrm{l}$ of this mixture was pipetted onto the third and fourth channels on the chip. Lastly, $4 \mu 1$ of the $100 \mathrm{ng}$ sample solution was mixed with $40 \mu \mathrm{l}$ of sterile PBS, and $22 \mu \mathrm{l}$ of this mixture was pipetted onto both the fifth and sixth channels on the chip. After 10 minutes, $20 \mu l$ of sterile PBS was added to all seven channels. After another 15 minutes, the top layer of the chip was spun in the housing to line up with the adjacent, dry channels available on the second layer. Then, $20 \mu \mathrm{l}$ of sterile PBS was pipetted directly onto the detection region of each channel. Lastly, after 10 more minutes, the chip was removed from the housing and $5 \mu 1$ of 1Step Ultra TMB-ELISA substrate solution was added to each detection region. Images were taken of the chip using an iPhone 5. This same procedure was then repeated for the second prepared 3D chip. 


\section{Troubleshooting Experiments}

Due to the poor results of the initial ELISA test, several experiments were run to pinpoint what went wrong. First, a test was performed to see if the detection antibody was able to be dried onto the conjugate pad successfully. This test was also used to compare results between a conjugate pad that was sitting on regular paper and a conjugate pad that was sitting on waxprinted paper during the drying process. Next, three different tests were run on a single chip to observe the effects of the conjugate pad and the BSA blocking step on ELISA results. Each test was performed on two channels ( 6 channels total) and one channel was used as a control. These four experiments are described in the following sections.

\section{Detection Antibody Storage on Conjugate Pad}

In this first test, two conjugate pads were used with one sitting atop a sheet of regular printer paper and one sitting atop wax-printed cellulose paper. Only one channel was used on each conjugate pad. This experiment was performed using the following procedure:

- Measure out $5 \mu \mathrm{l}$ of the $0.9 \mathrm{mg} / \mathrm{ml}$ stock solution of HRP-conjugated detection antibody

- Add $85 \mu 1$ of DI water to the solution (producing a $50 \mathrm{ng} / \mu 1$ solution)

- Mix $4 \mu 1$ of this solution with $40 \mu 1$ of DI water

- Pipette $22 \mu 1$ of the diluted solution onto one channel on each chip

- Dry both chips in a humidity chamber for 10 minutes and then an additional $10-20$ minutes with vacuum pump on until visibly dry

- Add $5 \mu \mathrm{l}$ of TMB substrate onto each channel and observe for a color change The color change on both chips was then analyzed to determine how successful each chip was in storing the HRP-conjugated detection antibody. 
Inclusion vs. Exclusion of Conjugate Pad

To determine if the conjugate pad was causing issues during the ELISA, tests were run with and without the conjugate pad in the 3D chip for comparison. Both of these tests were run on a single chip, each utilizing two channels on the chip. For the test without the conjugate pad incorporated into the chip, the HRP-conjugated antibody was mixed directly with the troponin solution before being introduced to the chip. For both tests, two concentrations of troponin were prepared. These solutions were prepared using the following procedures:

$1 \mu \mathrm{g}$ Troponin Solution

- Mix $3 \mu 1$ of the $1.93 \mu \mathrm{g} / \mu 1$ stock solution of troponin with $8.58 \mu 1$ of DI water

- Mix $10 \mu l$ of this solution with $90 \mu l$ of DI water

100 ng Troponin Solution

- Mix $10 \mu \mathrm{l}$ of the $1 \mu \mathrm{g}$ troponin solution with $90 \mu \mathrm{l}$ of DI water

Once these troponin solutions were made, the full ELISA test was run without the conjugate pad. The following procedure was used for this test:

- Mix $6 \mu 1$ of streptavidin with $40 \mu 1$ of DI water

- Pipette $23 \mu \mathrm{l}$ of this solution onto the first two channels of the nitrocellulose layer

- Dry the chip in a humidity chamber for 10 minutes and then an additional 20 minutes with the vacuum pump on

- Let the chip sit at room temperature until visibly dry

- Mix $2 \mu 1$ of the biotinylated $2 \mathrm{mg} / \mathrm{ml}$ capture antibody solution with $30 \mu \mathrm{l}$ of DI water

- Pipette $4 \mu \mathrm{l}$ of this solution onto the two channels on the nitrocellulose layer

- Dry the chip in a humidity chamber for 10 minutes and then an additional 2 minutes with vacuum pump on 
- Pipette $50 \mu \mathrm{l}$ of BSA dilution $(100 \mu \mathrm{l} / \mathrm{ml})$ onto both channels and let sit for 20 minutes

- Pipette $50 \mu \mathrm{l}$ of PBST onto each channel and let sit for 15 minutes

- Repeat this PBST wash step

- Dry chip in a humidity chamber with vacuum on until visibly dry

- Assemble the 3D chip (excluding the conjugate pad) and place in the housing

- Mix $2 \mu 1$ of the diluted HRP-conjugated detection antibody solution (from previous test) with $20 \mu 1$ of DI water

- Add $2 \mu l$ of the $100 \mathrm{ng}$ troponin solution into the diluted detection antibody solution and pipette the mixture onto the first channel of the 3D chip

- Repeat this process using $2 \mu \mathrm{l}$ of the $1 \mu \mathrm{g}$ troponin solution and pipette the mixture onto the second channel

- After 10 minutes, wash with $20 \mu$ l of DI water

- After another 15 minutes, spin the top layer of the chip once and wash with another $20 \mu 1$ of DI water

- After 10 more minutes, add $5 \mu$ of the TMB substrate to both channels While this test was being performed, another test involving the conjugate pad was being performed on the third and fourth channels of this chip simultaneously. This test essentially followed the same procedure as the one described above, except that it included the conjugate pad instead of premixing the HRP-conjugated antibody and troponin solutions. The conjugate pad was prepared using the following procedure:

- Mix $4 \mu \mathrm{l}$ of the diluted detection antibody solution (from the detection antibody storage test) with $40 \mu \mathrm{l}$ of DI water 
- Pipette $22 \mu 1$ of this solution onto the conjugate pad designated for the third and fourth channels on the chip

- Dry the conjugate pad in humidity chamber for 10 minutes and then with the vacuum pump on until visibly dry

Once the conjugate pad was prepared and incorporated into the 3D chip, the chip was assembled in the housing. The different troponin solutions were then pipetted onto the third and fourth channels of the chip (containing the conjugate pad). First, $2 \mu \mathrm{l}$ of the $100 \mathrm{ng}$ troponin solution was mixed with $20 \mu 1$ of DI water and pipetted onto the third channel of the chip. Then, $2 \mu \mathrm{l}$ of the $1 \mu \mathrm{g}$ troponin solution was mixed with $20 \mu \mathrm{l}$ of DI water and pipetted onto the fourth channel of the chip. The same wash steps as described above were then performed and finally 5 $\mu 1$ of TMB substrate was added to both channels. The results of the first test (without the conjugate pad) and the second test (with the conjugate pad) were then compared.

\section{BSA Blocking vs. No Blocking}

To determine if blocking the nitrocellulose membrane with BSA has an effect on the signal, a last test was performed without the BSA blocking step. For this test, the fifth and sixth channels of the chip were prepared using the same procedure described for the first and second channels, except without the BSA blocking step and PBST wash step. Once the streptavidin and capture antibody were dried onto the nitrocellulose layer, the layer was incorporated into the 3D chip without being blocked by BSA or washed with PBST. Using the same procedure described above, the HRP-conjugated detection antibody and troponin solutions were mixed and applied directly to the 3D chip onto the fifth and sixth channels. This test also excluded the conjugate pad. The same wash steps were then performed and the TMB substrate was added. 
Since this test was identical to the test performed on the first and second channels of the chip, minus the BSA blocking and PBST washing steps, the results of the two tests were compared to see if blocking with BSA had an impact on the signal.

\section{$\underline{\text { Final ELISA Test }}$}

After finalizing the ELISA protocol, one final test was performed on a single chip. Six channels were designated three different troponin masses ( 2 channels per mass) and one channel was used as a negative control. This test followed the same procedure as described above for the ELISA run without a conjugate pad in the Inclusion vs Exclusion of Conjugate Pad experiment. However, the volumes used for preparing the streptavidin solution were multiplied by three to accommodate for six channels instead of just two. Also, an additional troponin solution was made to test for a total of three troponin masses. The three different troponin solutions contained $1 \mu \mathrm{g}, 100 \mathrm{ng}$, and $10 \mathrm{ng}$ of troponin, respectively. The $1 \mu \mathrm{g}$ and $100 \mathrm{ng}$ solutions were made using the same procedure as described above in the Inclusion vs Exclusion of Conjugate Pad experiment. The $10 \mathrm{ng}$ solution was made using the following procedure:

- Mix $10 \mu \mathrm{l}$ of the $100 \mathrm{ng}$ solution with $90 \mu \mathrm{l}$ of DI water

The same procedure used in the previous test mentioned above was then used to apply each HRP-conjugated detection antibody + troponin solution to each detection spot. However, the volumes used to prepare these mixed solutions were doubled to accommodate for two channels per troponin mass instead of just one. The following wash steps and addition of TMB substrate were also performed in the same manner as described in the previous experiment without a conjugate pad. Cell phone images were then taken of the chip using an iPhone 5 camera. The app Scannable was also used to help adjust for poor lighting while taking the pictures. 


\section{Data Analysis}

The ELISA test images were run through a custom Matlab code that was written by Erik Huynh [36]. This code was able to assign color intensity values to each detection region. These values were directly correlated with the amount of troponin present in each region. 


\section{RESULTS}

\section{Dye Testing}

\section{Fluid Connectivity}

The results from dye testing performed on the first circular chip in its housing with the screws tightened in place can be seen in Figure 22.

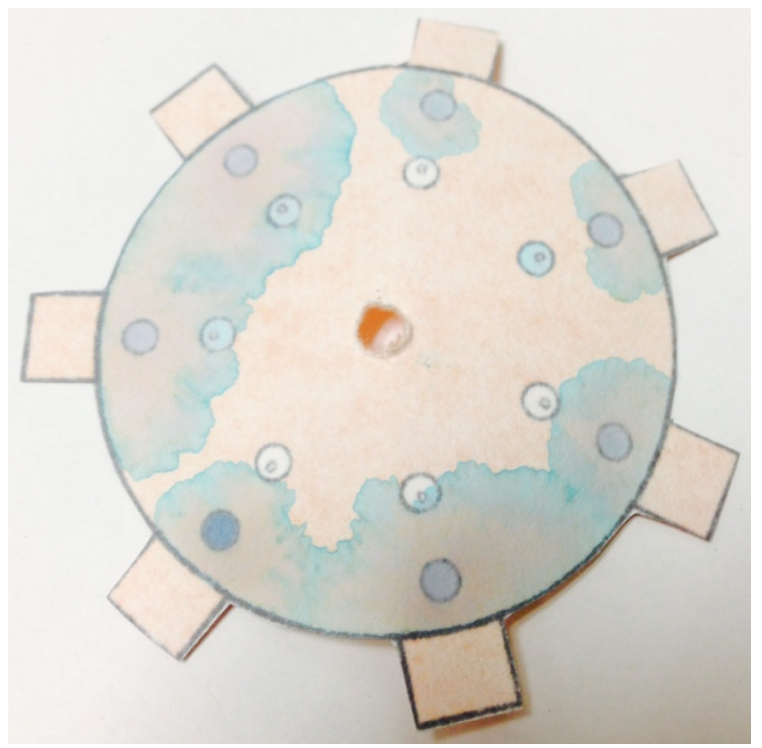

Figure 22: Dye Testing Results for First Circular Chip Positioned in the Housing Assembled with Screws

Due to the unsatisfactory results of the first dye test, the housing was instead held together by paper clamps in hope of applying more pressure to the first circular chip. The results of this dye test can be seen in Figure 23. 


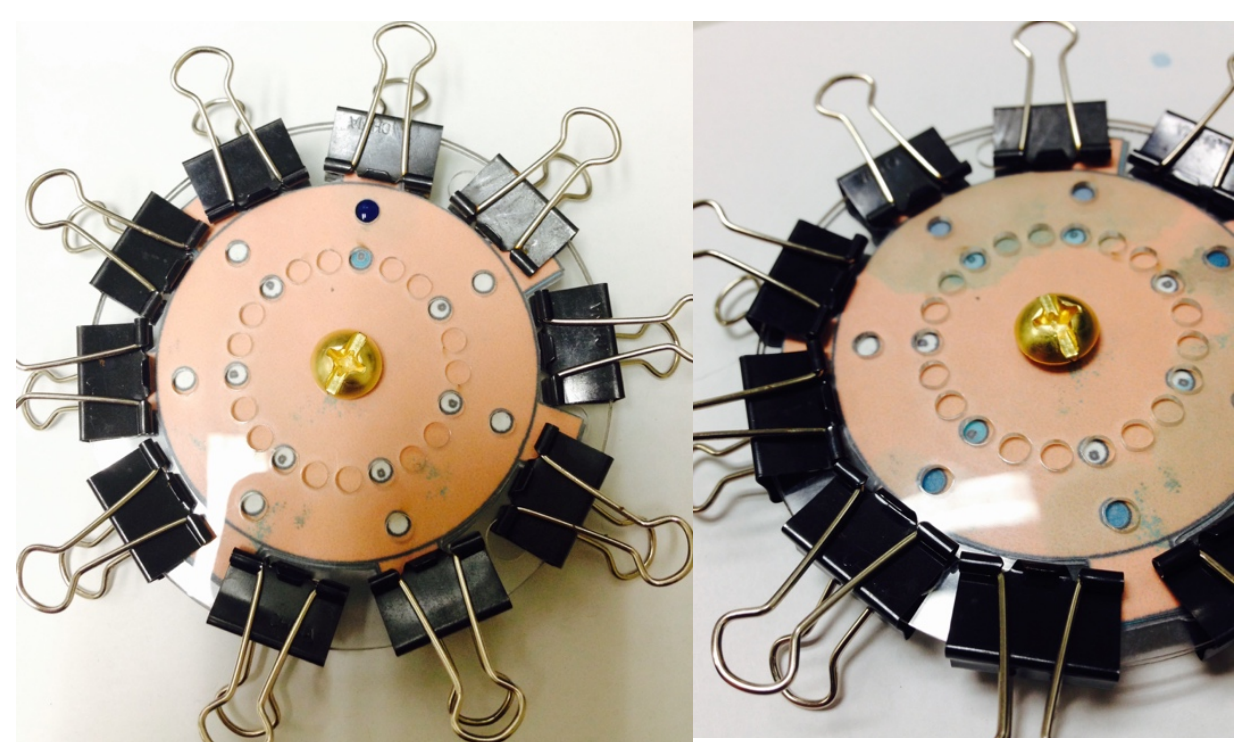

Figure 23: Dye Testing Results for the First Circular Chip Positioned in the Housing Assembled with Paper Clamps

As seen in Figure 23, some of the channels (left image) were successful in keeping the fluid within the channel boundaries while some (right image) were unsuccessful. This dye test was still considered a failure, which lead to the production of a new chip and housing. The results of dye testing with this new chip and housing can be seen in Figure 24.
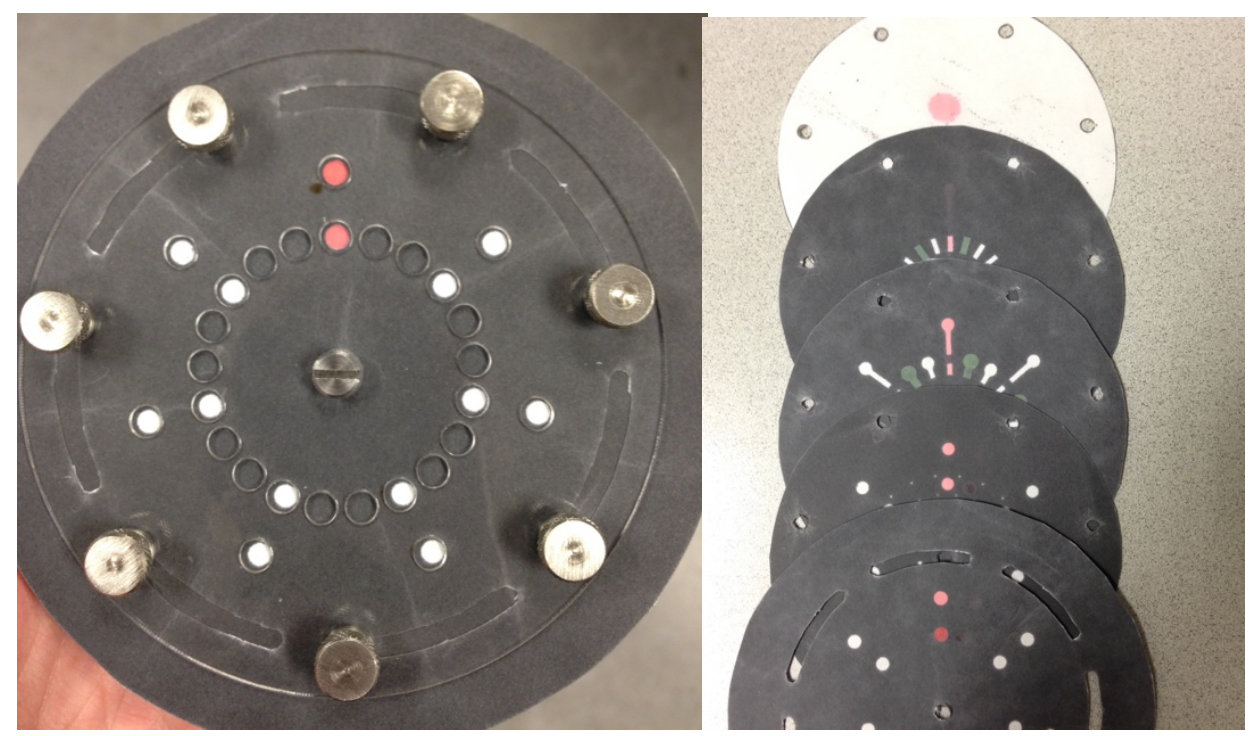

Figure 24: Dye Testing Results for the Final Chip Positioned in the Final Housing 
As seen in Figure 24, the final chip and housing successfully routed fluid within the appropriate channels. Due to these satisfactory results, the final chip and housing were used for all future testing.

\section{Conjugate Pad}

Once the chip and housing were finalized, dye testing with the conjugate pad was performed. The first test performed involved the conjugate pad composed of cellulose paper. The results of this dye test can be seen in Figure 25.

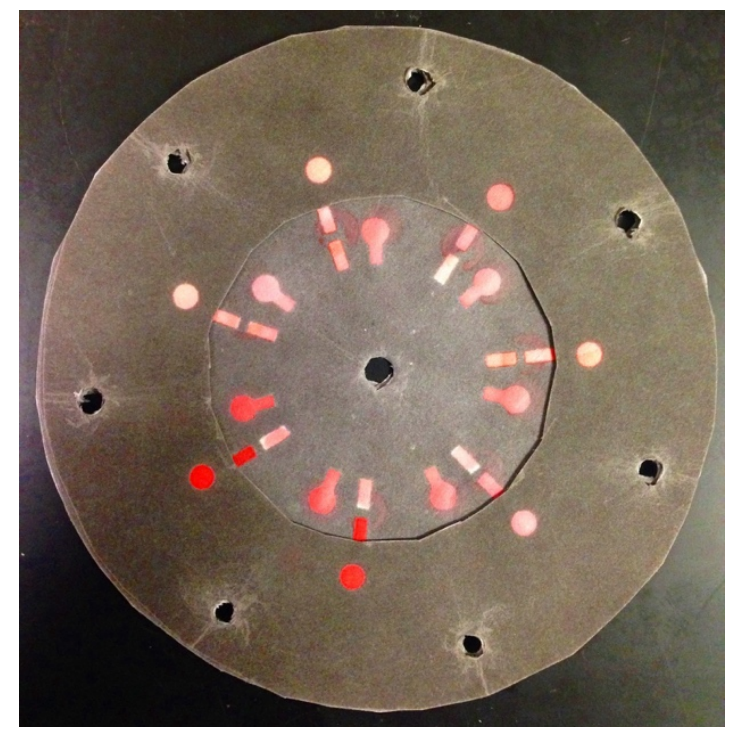

Figure 25: Dye Testing Results for Chip Utilizing the Conjugate Pad Made with rallulno Domar

As can be seen in Figure 25, this test was a success because the dye dried onto the conjugate pad (top layer in the image) was successful wicked throughout the rest of the chip (bottom layers in the image) with the introduction of DI water.

The next dye test performed involved the conjugate pad made of fiberglass. The results of this dye test can be seen in Figure 26. 


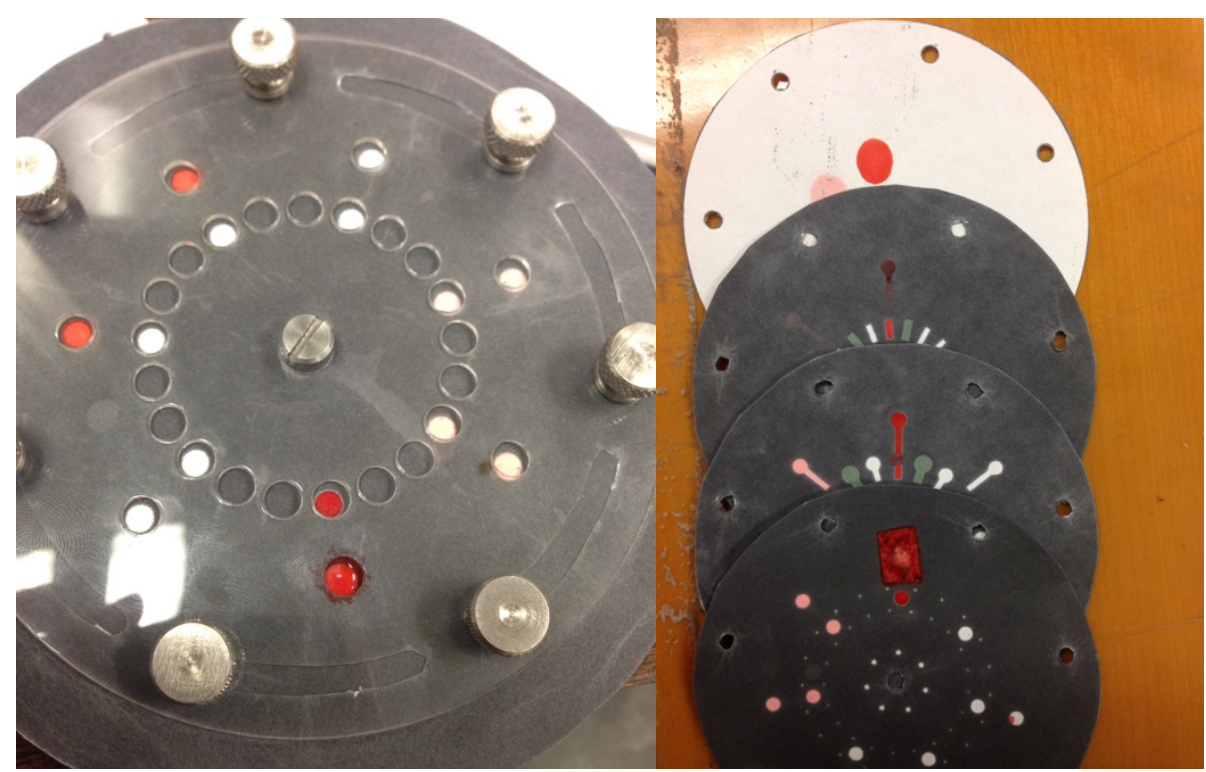

Figure 26: Dye Testing Results for the Chip Utilizing the Conjugate Pad Made with Fiberglass

As seen in Figure 26, this dye test was also a success since the red dye was able to resolubilize from the fiberglass conjugate pad (attached to the top layer in the right image) and wick through the remainder of the chip in the designated channel. However, due to the amount of dye lost within the conjugate pad area itself, the conjugate pad made of cellulose paper was used for all future tests.

\section{Nitrocellulose Detection Layer}

Once the conjugate pad selection was finalized, dye testing was performed with the nitrocellulose layer incorporated into the chip. The results of this testing can be seen in Figure 27. 


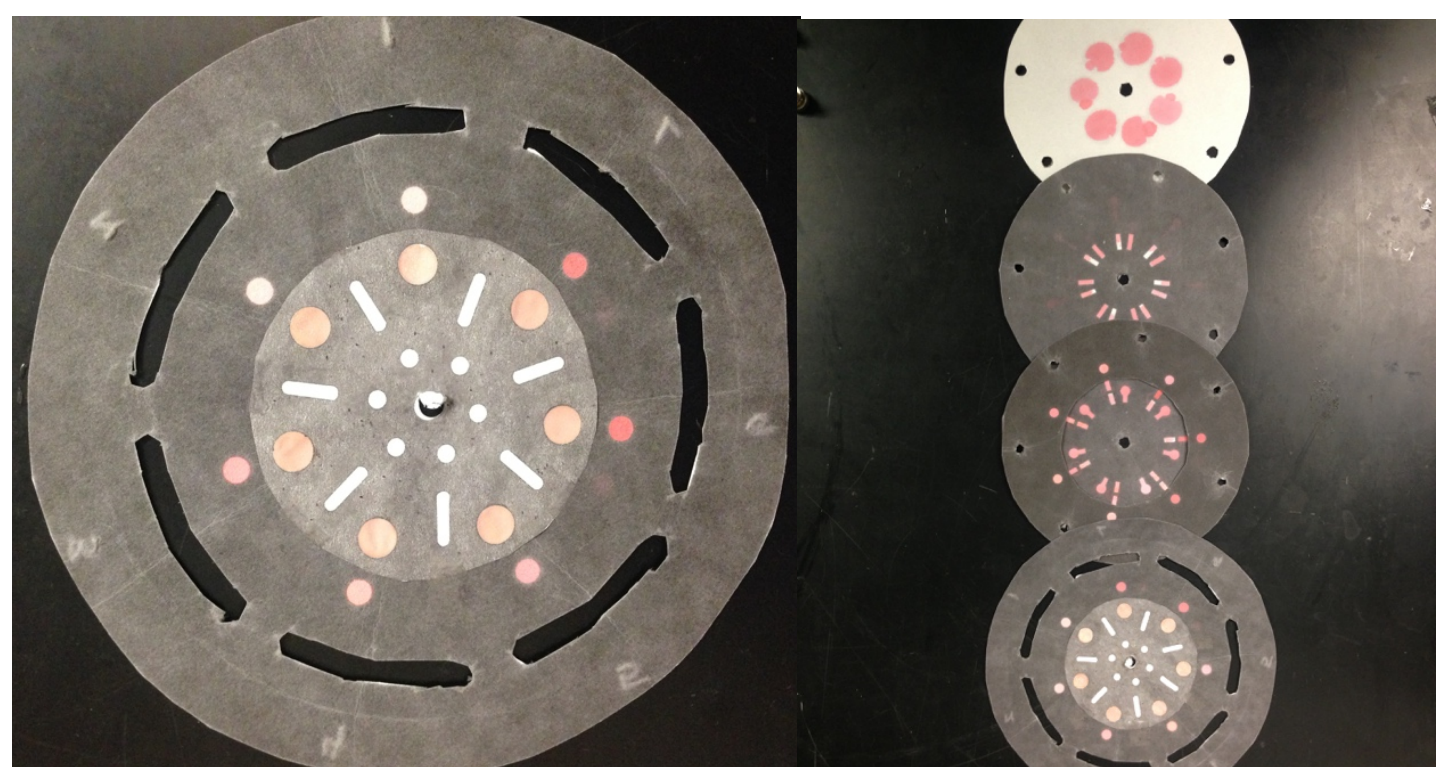

Figure 27: Dye Testing Results for the Chip with the Nitrocellulose Layer Included

As can be seen in Figure 27, the nitrocellulose layer, which was taped to the top layer of the chip (left image), was successfully incorporated into the 3D chip. With both the conjugate pad and nitrocellulose layer included, the 3D chip was able to wick the fluid between layers without it leaving the designated channels.

\section{Development Time Testing}

The development times for a fully assembled chip can be found in Table 1 .

Table 1: Development Times

\begin{tabular}{|l|l|l|l|}
\hline Test & $\begin{array}{l}\text { Time to Detection } \\
\text { Region }\end{array}$ & Time to Absorbent Pad & $\begin{array}{l}\text { Time to Absorbent } \\
\text { pad (Wash step) }\end{array}$ \\
\hline 1 & $0: 24.18$ & $1: 37.64$ & $0: 23.95$ \\
\hline 2 & $0: 14.56$ & $3: 20.68$ & $0: 31.50$ \\
\hline 3 & $0: 9.98$ & $7: 25.04$ & $0: 03.71$ \\
\hline 4 & $0: 14.16$ & $1: 44.15$ & $0: 05.05$ \\
\hline 5 & $0: 17.25$ & $5: 51.55$ & $0: 04.96$ \\
\hline 6 & $0: 58.00$ & $3: 46.05$ & $0: 05.71$ \\
\hline 7 & $0: 29.85$ & $1: 53.18$ & $0: 04.33$ \\
\hline
\end{tabular}


Each test was performed on a single channel on the chip ( 7 total). $50 \mu \mathrm{l}$ of DI water was

used for each test, as well as an additional $50 \mu \mathrm{l}$ for the wash step. The means and standard deviations of each of the development times can be found in Table 2 .

Table 2: Means and Standard Deviations for Development Times

\begin{tabular}{|l|l|l|l|l|l|}
\hline $\begin{array}{l}\text { Mean Time } \\
\text { to Detection } \\
\text { Region }\end{array}$ & $\begin{array}{l}\text { Standard } \\
\text { Deviation for } \\
\text { Time to } \\
\text { Detection } \\
\text { Region }\end{array}$ & $\begin{array}{l}\text { Mean Time } \\
\text { to Absorbent } \\
\text { Pad }\end{array}$ & $\begin{array}{l}\text { Standard } \\
\text { Deviation for } \\
\text { Time to } \\
\text { Absorbent } \\
\text { Pad }\end{array}$ & $\begin{array}{l}\text { Mean Time } \\
\text { to Absorbent } \\
\text { Pad (Wash } \\
\text { step) }\end{array}$ & $\begin{array}{l}\text { Standard } \\
\text { Deviation for } \\
\text { Time to } \\
\text { Absorbent } \\
\text { Pad (Wash } \\
\text { step) }\end{array}$ \\
\hline $0: 23.99$ & $0: 15.20$ & $3: 39.76$ & $2: 04.30$ & $0: 11.32$ & $0: 10.59$ \\
\hline
\end{tabular}

\section{Antibody Experiments: 3D Paper Microfluidic Device}

\section{$\underline{\text { Initial ELISA Test }}$}

The first ELISA test was performed on two separate chips. This test was unsuccessful because no color change was seen on either chip when the TMB substrate was added to the detection regions. These results can be seen in Figure 28.

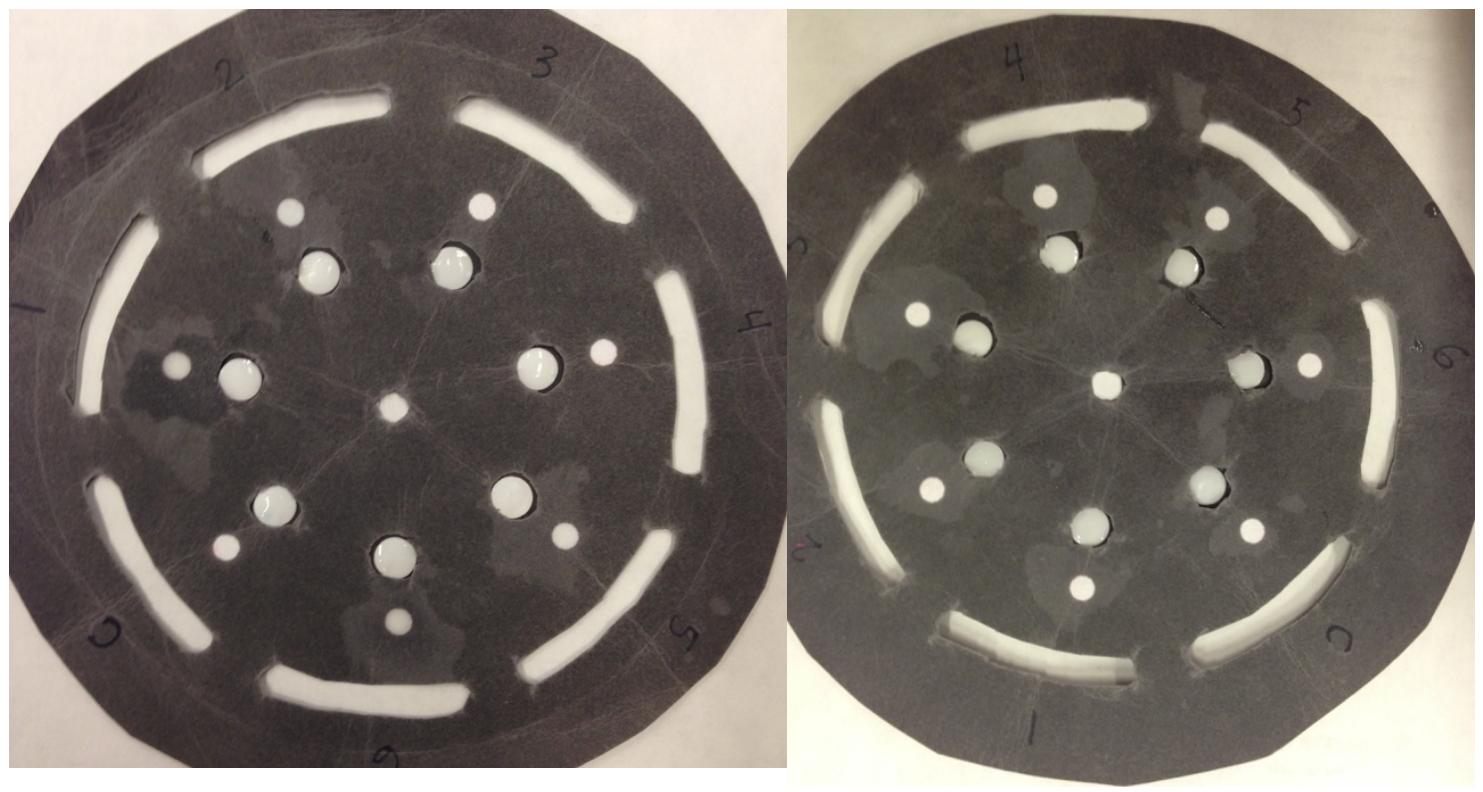

Figure 28: Results for First (left) and Second (right) Final Chips Used in the First ELISA Test 
As can be seen in Figure 28, each detection region remained a clear color similar to the negative controls on each chip, indicating a negative signal. To quantitatively analyze these results, the images were run through a custom Matlab code [36]. This code analyzes each detection region on the chip and assigns a color intensity value, with higher values representing a more intense signal. The Matlab results for the first chip can be seen in Figure 29.

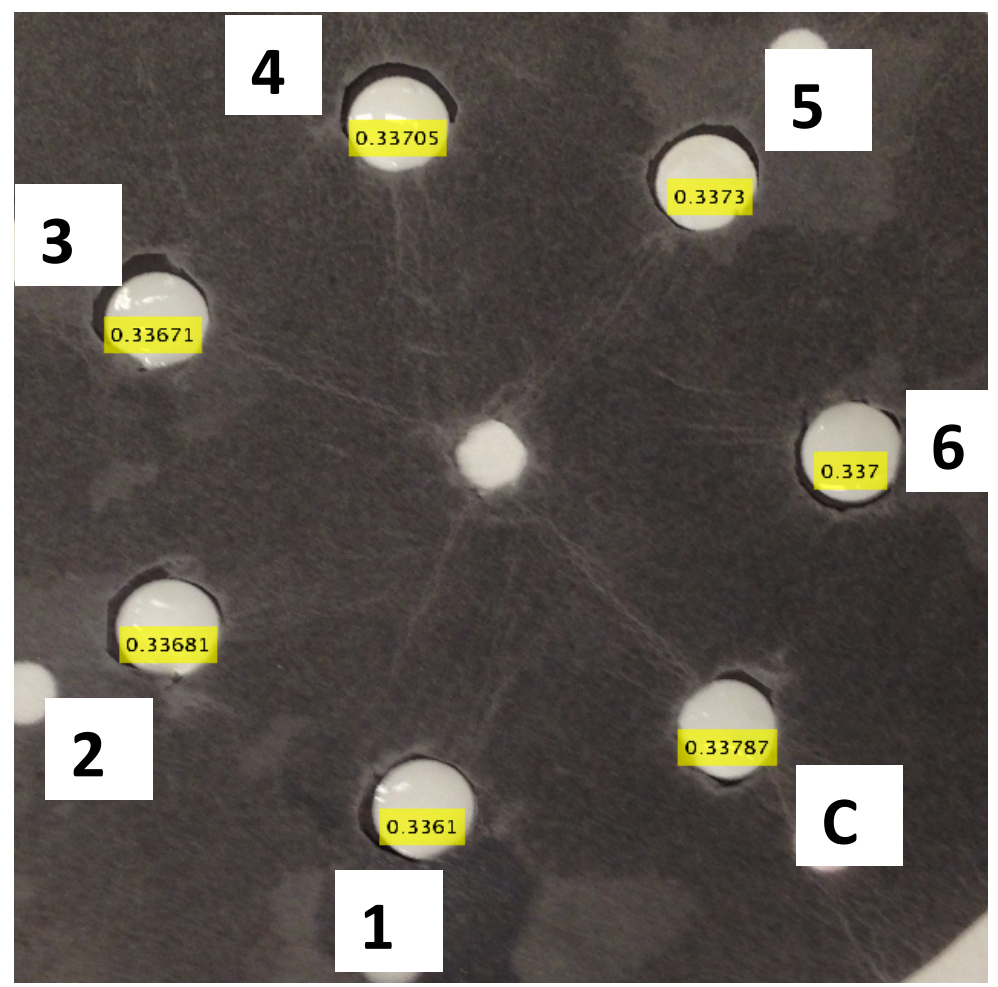

Figure 29: Quantitative Results for the First Chip Used in the First ELISA Test Obtained Using Custom Matlab Code

As seen in Figure 29, the control detection region (labeled C) has almost exactly the same color intensity value (about 0.33 ) as the rest of the detection regions. This proves that channels 1 through 6 have a negative signal since their color intensities do not change relative to the control channel when the TMB substrate is added. Similar results were seen for the second chip in this experiment. These results can be seen in Figure 30. 


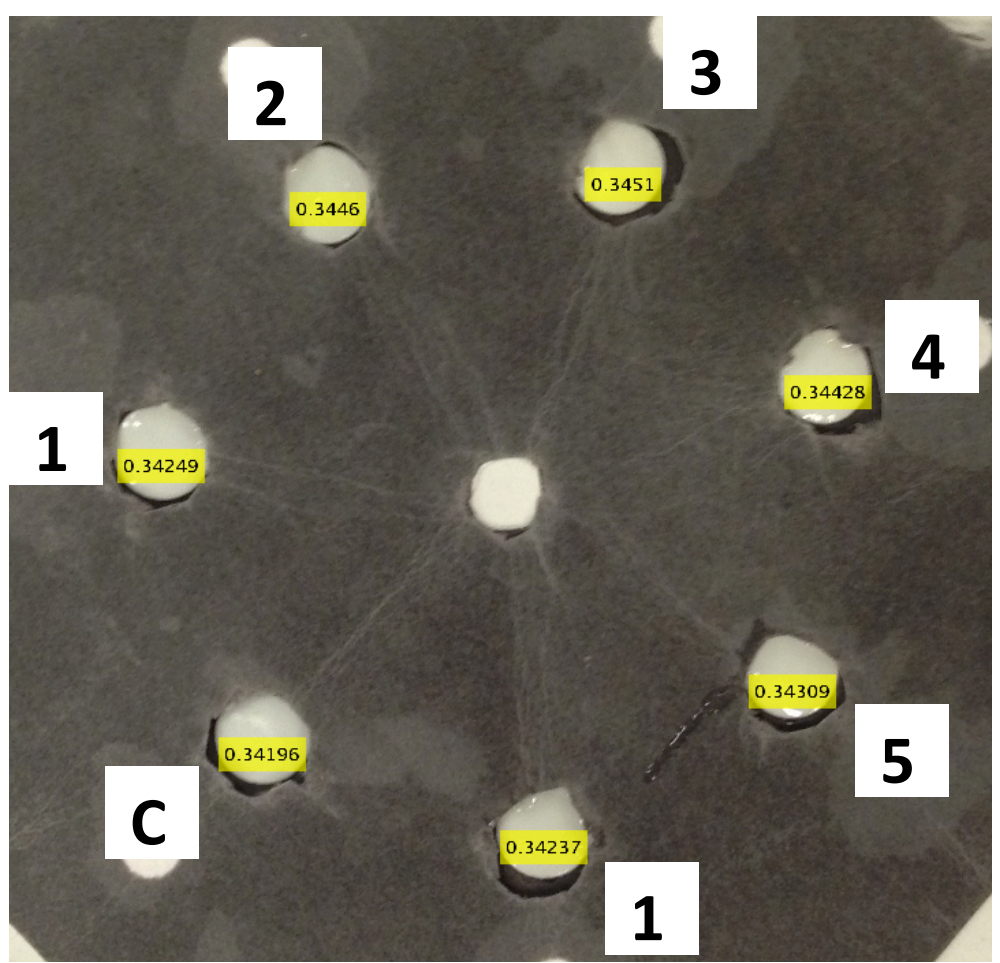

Figure 30: Quantitative Results for the Second Chip Used in the First ELISA Test Obtained Using Custom Matlab Code

As seen in Figure 30, the control detection region (labeled C) has almost exactly the same color intensity value as the rest of the detection regions (about 0.34 ). Due to the negative signal seen in channels 1 through 6 once again, it was concluded that the first ELISA test was a failure. As a result, troubleshooting experiments were run to determine what went wrong.

\section{Troubleshooting Experiments}

\section{Detection Antibody Storage on Conjugate Pad}

To confirm that the cellulose conjugate pad is able to store detection antibody and to compare results between a chip sitting atop regular paper and a chip sitting atop wax-patterned cellulose paper, detection antibody was dried onto two separate chips. The results of this test can be seen in Figure 31. 


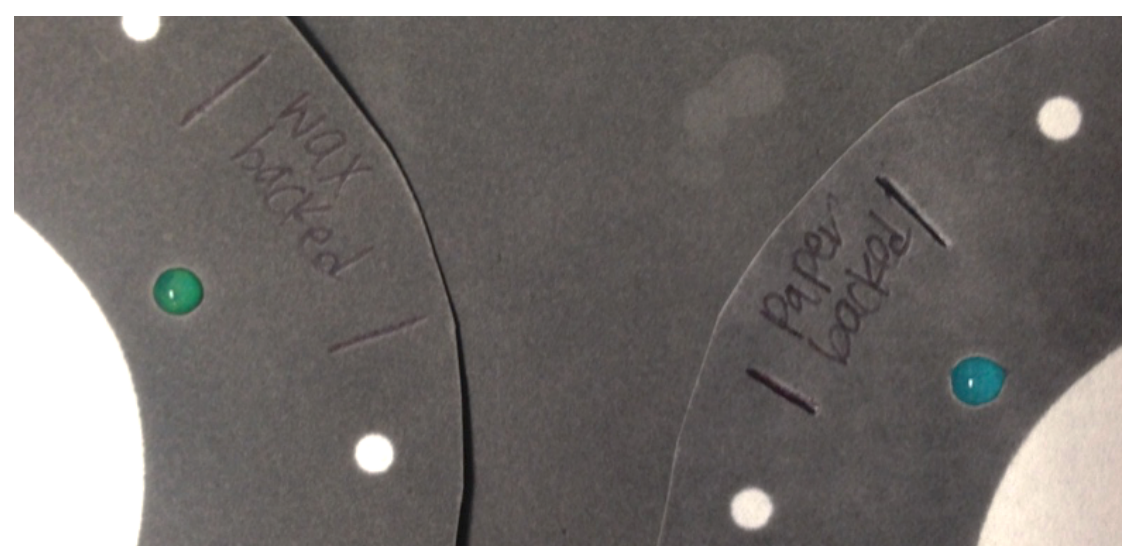

Figure 31: Results for Wax-backed (left) and Paper-backed (right) Chips Used in the Detection Antibody Storage on Conjugate Pad Experiment

The chip on the left was sitting atop wax-patterned paper, while the chip on the right was sitting atop regular paper. As seen in Figure 31, both chips showed a positive signal, indicating that the detection antibody was successfully dried on both. However, the chip sitting atop waxpatterned paper showed a green color change while the chip sitting atop regular paper showed a blue color change. This difference in color indicated that the two chips had different amounts of detection antibody stored on them. The reason for this will be further discussed in chapter 4 . To quantitatively analyze these results, the image was run through custom Matlab code [36]. These results can be seen in Figure 32 . 

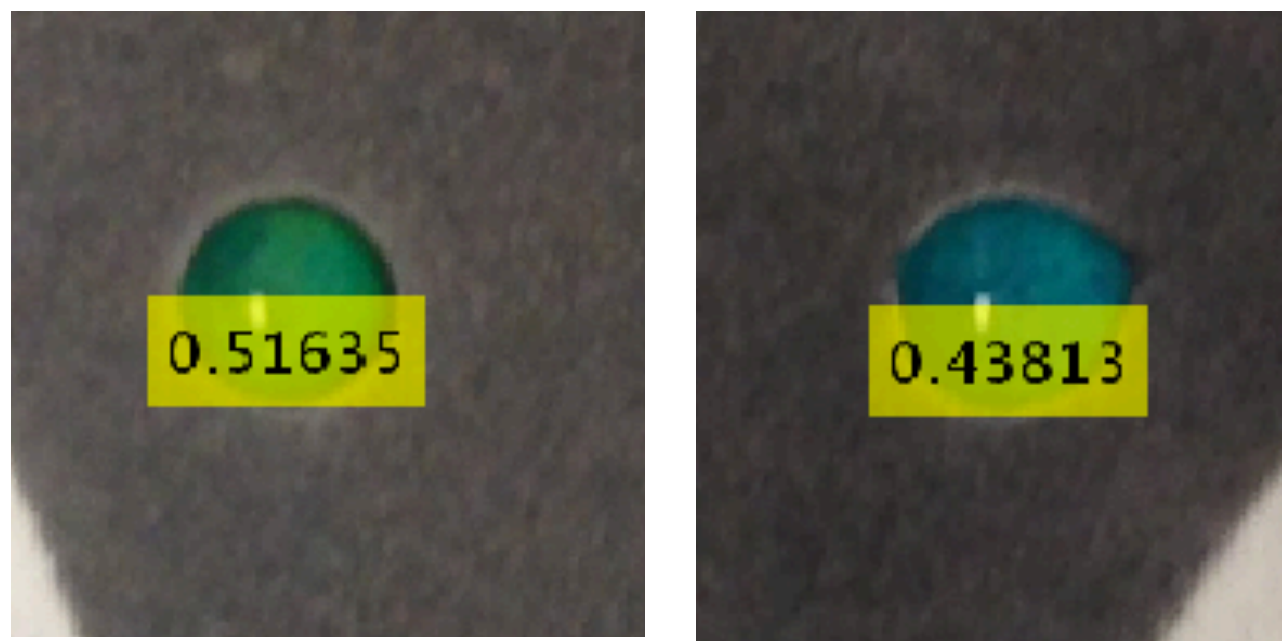

Figure 32: Quantitative Results for Wax-backed (left) and Paper-backed (right) Chips Used in the Detection Antibody Storage on Conjugate Pad Experiment Obtained Using Custom Matlab Code

As seen in Figure 32, the wax-backed chip (left image) had a higher color intensity value (0.51635) than the paper-backed chip (0.43813). This further supported the conclusion that the wax-backed chip was able to store more detection antibody than the paper-backed chip. Due to this finding, all chips used in future experiments were prepared atop a wax-patterned cellulose paper to maximize the amount of reagents stored on each chip.

\section{Inclusion vs. Exclusion of Conjugate Pad}

To determine if the conjugate pad was causing problems during the ELISA, a test was run to compare the results between two 3D chips arrangements: one that included the conjugate pad and one that excluded the conjugate pad. The test was run on a single chip, with two channels assigned to each 3D chip arrangement. One channel was used as a control (labeled C), two channels excluded conjugate pad (labeled 1 and 2), and two channels included the conjugate pad (labeled 3 and 4). Furthermore, channel 1 and 3 used 100 ng of troponin while channels 2 and 4 used $1 \mu \mathrm{g}$ of troponin. These results can be seen in Figure 33. 


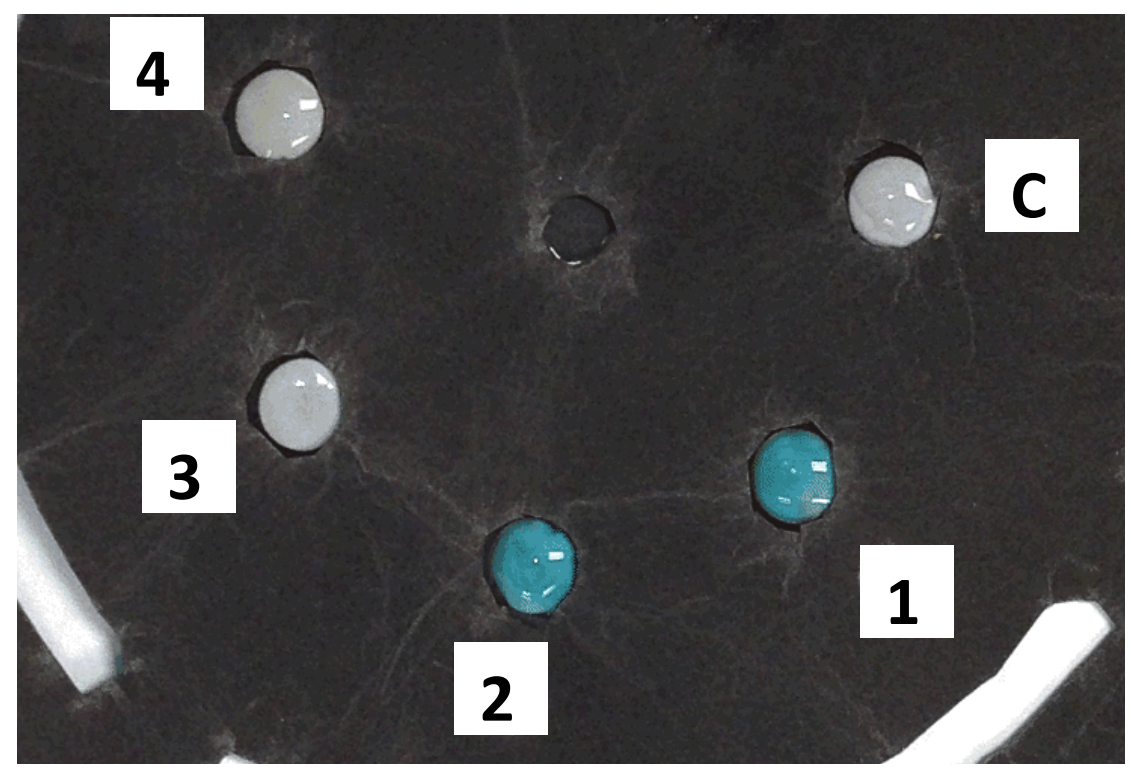

Figure 33: Results for the Inclusion vs. Exclusion of Conjugate Pad Experiment with Channel $\mathrm{C}$ a control, Channels 1 and 2 Excluding the Conjugate Pad, and Channels 3 and 4 Including the Conjugate Pad

As seen in Figure 33, channels 1 and 2 showed a color change while channels 3 and 4 remained a clear color similar to the negative control. This indicated that only channels 1 and 2 were showing a positive signal. Since identical masses of troponin were used in the two channel pairs $(1,2$ and 3,4$)$, they were expected to have similar color changes. To quantitatively analyze these results, this image was run through custom Matlab code [36]. These results can be seen in Figure 34. 


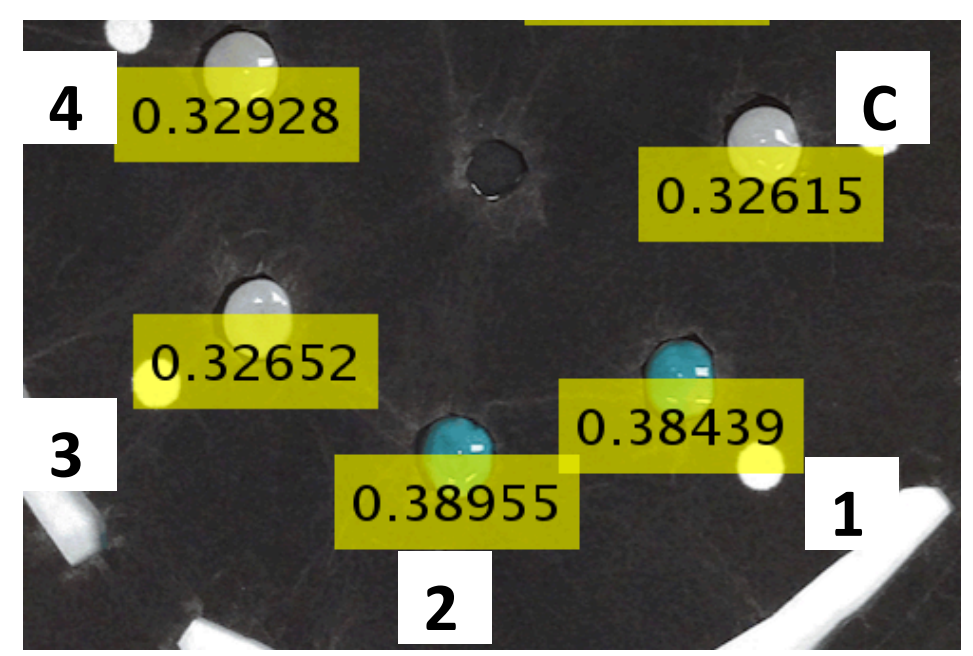

Figure 34: Quantitative Results for the Inclusion vs. Exclusion of Conjugate Pad Experiment Obtained Using Custom Matlab Code. Channel $\mathrm{C}$ Used as a control, Channels 1 and 2 Excluded the Conjugate Pad, and Channels 3 and 4 Included the Conjugate Pad.

As seen in Figure 34, channels with the conjugate pad included (3 and 4) had almost identical color intensity values as the control channel (0.32), indicating a negative signal. The channels that excluded the conjugate pad (1 and 2) had higher color intensity values than the control (0.38), indicating a positive signal. Due to the apparent issues with inclusion of the conjugate pad, the conjugate pad was excluded in all future testing to maximize the detection signal.

\section{BSA Blocking vs. No Blocking}

To determine if blocking with BSA has an effect on the detection signal, a test was run to compare results between channels that were blocked with BSA and channels that were not blocked at all. The test was run on a single chip, with one negative control channel (labeled C), two BSA blocked channels (labeled 1 and 2), and two unblocked channels (labeled 3 and 4).

Channel 1 and 3 used 100 ng of troponin while channels 2 and 4 used $1 \mu \mathrm{g}$ of troponin. Furthermore, all channels did not include the conjugate pad. The results of this test can be seen in Figure 35. 


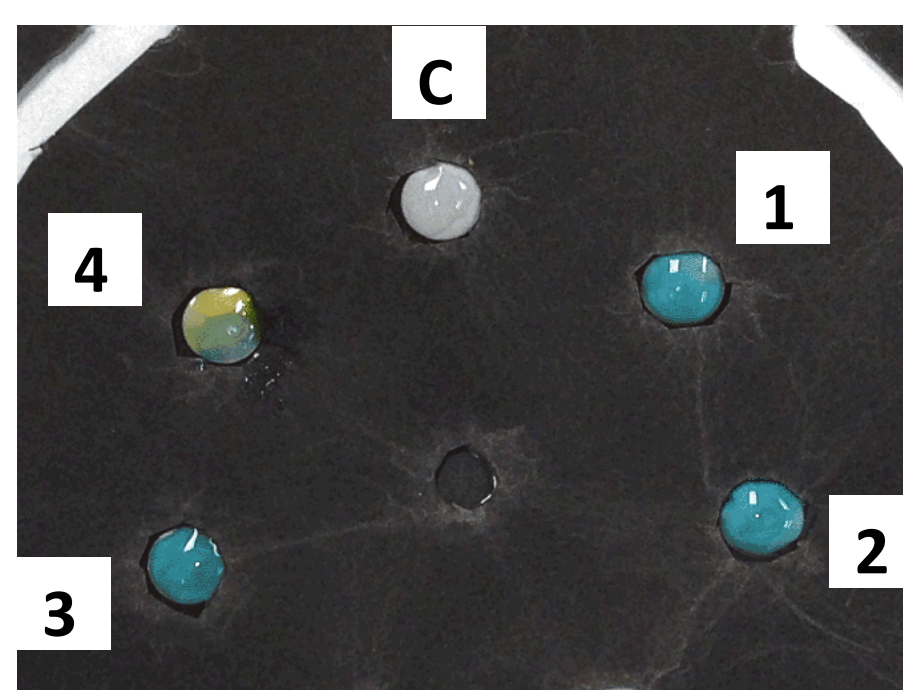

Figure 35: Results for the BSA Blocking vs. No Blocking Experiment with Channel $\mathrm{C}$ a Control, Channels 1 and 2 Blocked with BSA, and Channels 3 and 4 Unblocked

As seen in Figure 35, channels 1 through 4 all showed a color change relevant to the control channel, indicating a positive signal. However, channel 4 quickly turned from a blue color to a yellow color. Since the yellow color is considered a by product of the reaction between HRP and TMB substrate, a quicker transition to this yellow color indicates a larger concentration of HRP present. Since only channel 4 changed to a yellow color, it seems to have the highest concentration of HRP and thus the strongest signal. To quantitatively analyze these results, the image was run through custom Matlab code [36]. These results can be seen in Figure 36. 


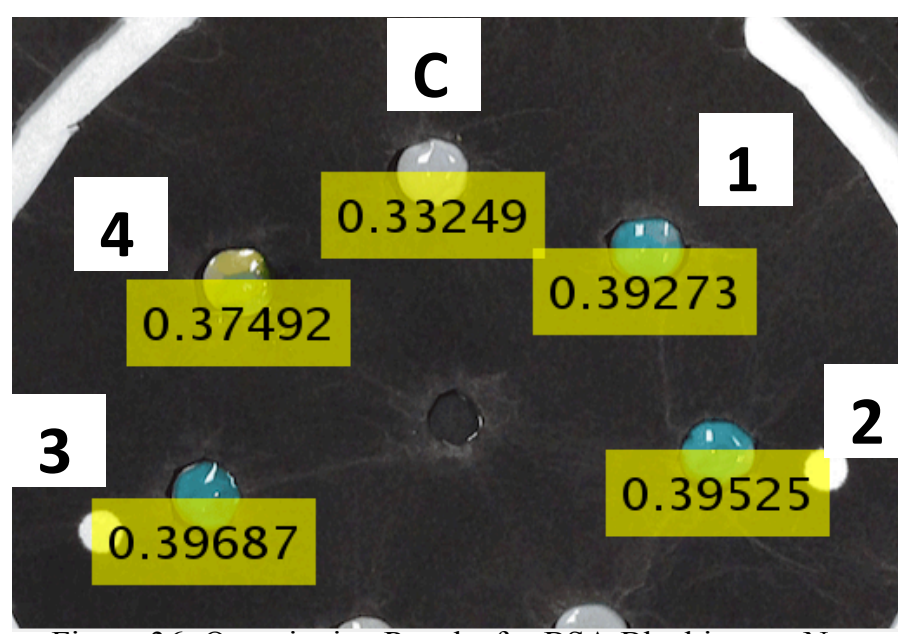

Figure 36: Quantitative Results for BSA Blocking vs. No Blocking Experiment Obtained Using Custom Matlab Code. Channel C Used as a Control, Channels 1 and 2 Blocked with BSA, and Channels 3 and 4 Unblocked.

As seen in Figure 36, channels 1, 2, and 3 all have similar color intensity values $(\sim 0.39)$. Channel 4, on the other hand, has a slightly lower color intensity value (0.37). Even though the yellow color indicates a higher concentration of HRP, the code is setup to analyze a blue color so it is unable to accurately analyze channel 4 . Overall, it appeared that the unblocked channels (3 and 4) had a stronger signal than the BSA blocked channels (1 and 2), even though both channel pairs had identical masses of troponin pipetted onto them. To prevent an enhanced signal due to non-specific binding of the detection antibody to the nitrocellulose detection layer, all future tests were performed with a BSA blocking step.

\section{$\underline{\text { Final ELISA Test }}$}

Once the ELISA protocol was finalized, a final test was run to compare the difference in signals between three different troponin masses: $10 \mathrm{ng}, 100 \mathrm{ng}$, and $1 \mu \mathrm{g}$. The results of this test can be seen in Figure 37. 


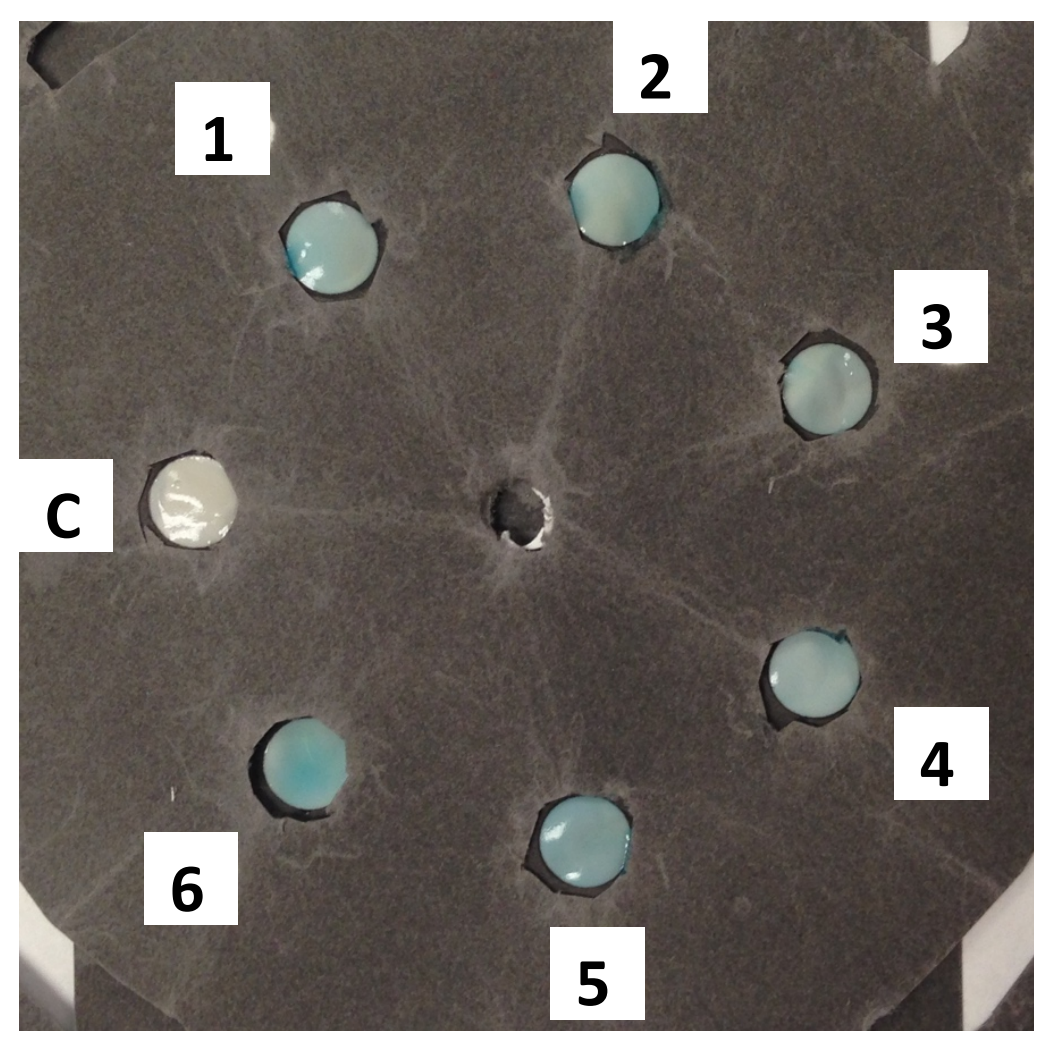

Figure 37: Results for the Final ELISA Test with Channel C a control, Channels 1 and 4 Using 10 ng Troponin, Channels 2 and

5 Using $100 \mathrm{ng}$ Troponin, and Channels 3 and 6 Using $1 \mu \mathrm{g}$ Troponin

As seen in Figure 37, channels 1 through 6 showed a color change relative to the negative control channel (labeled C). Channels 1 and 4 received 10 ng of troponin, channels 2 and 5 received $100 \mathrm{ng}$ of troponin, and channels 3 and 6 received $1 \mu \mathrm{g}$ of troponin. However, the color differences between these 6 channels was hard to see by eye. To quantitatively analyze these results, the image was run through custom Matlab code [36]. These results can be seen in Figure 38. 


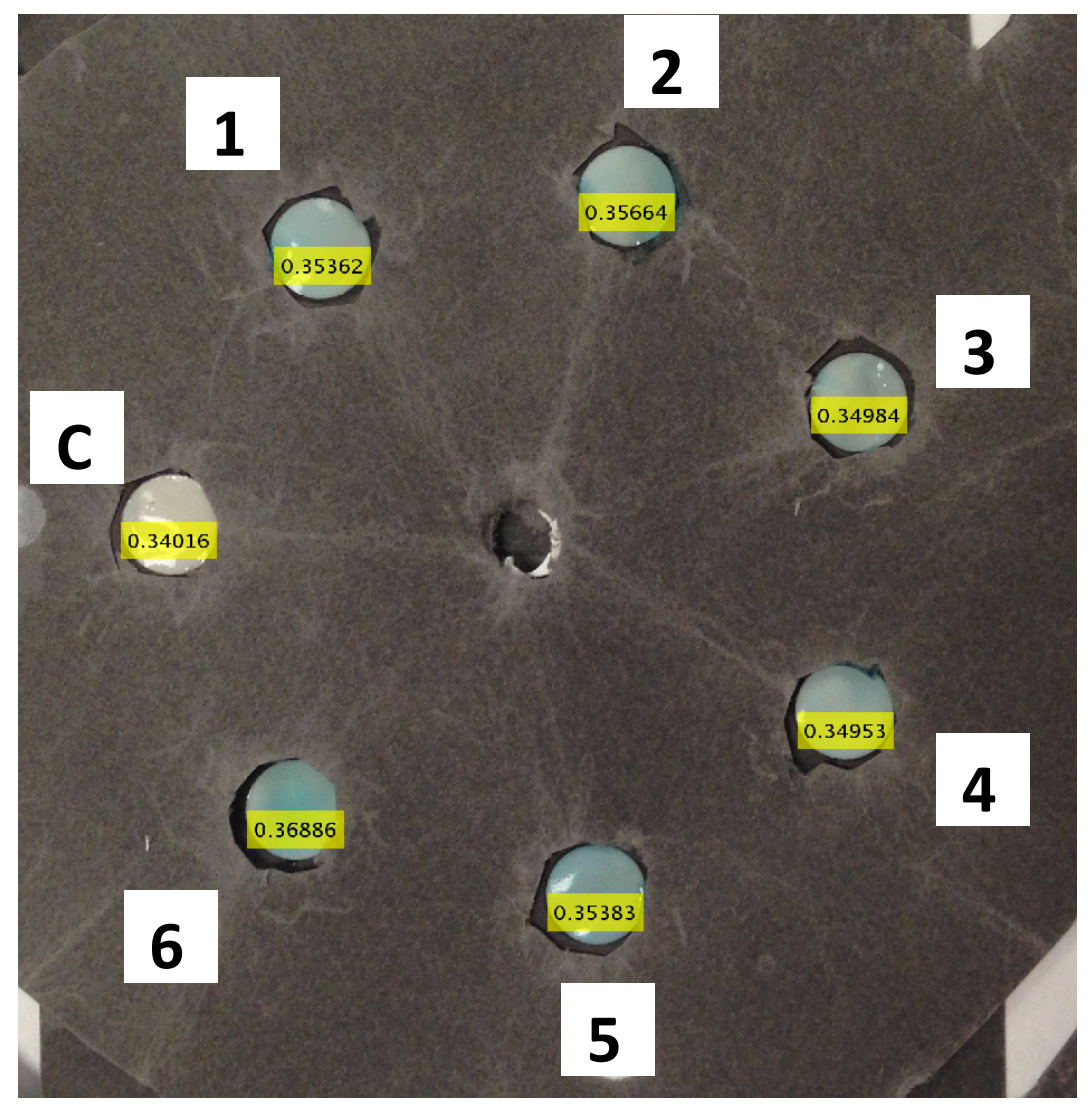

Figure 38: Quantitative Results for the Final ELISA Test Obtained Using Custom Matlab Code. Channel C Used as a control, Channels 1 and 4 Using $10 \mathrm{ng}$ Troponin, Channels 2 and 5 Using $100 \mathrm{ng}$ Troponin, and Channels 3 and 6 Using $1 \mu \mathrm{g}$ Troponin.

As seen in Figure 38, the six channels all had higher color intensity values $(>0.340)$ than the negative control channel. Channels 4 through 6 successfully showed a ladder effect in the signal intensity for the three troponin masses (10 ng in channel 4, $100 \mathrm{ng}$ in channel 5, and $1 \mu \mathrm{g}$ in channel 6). Channels 1 through 3 were not as successful at showing this same ladder effect since channel 3 (containing $1 \mu \mathrm{g}$ of troponin) had the smallest color intensity value. Possible explanations for this will be discussed in Chapter IIII. 
To compare this paper-based ELISA to a standard ELISA, the test was also run on a well plate. This test was performed by Jason Dierkhising, a biology student at Cal Poly San Luis Obispo. The results from this test can be seen in Figure 39.

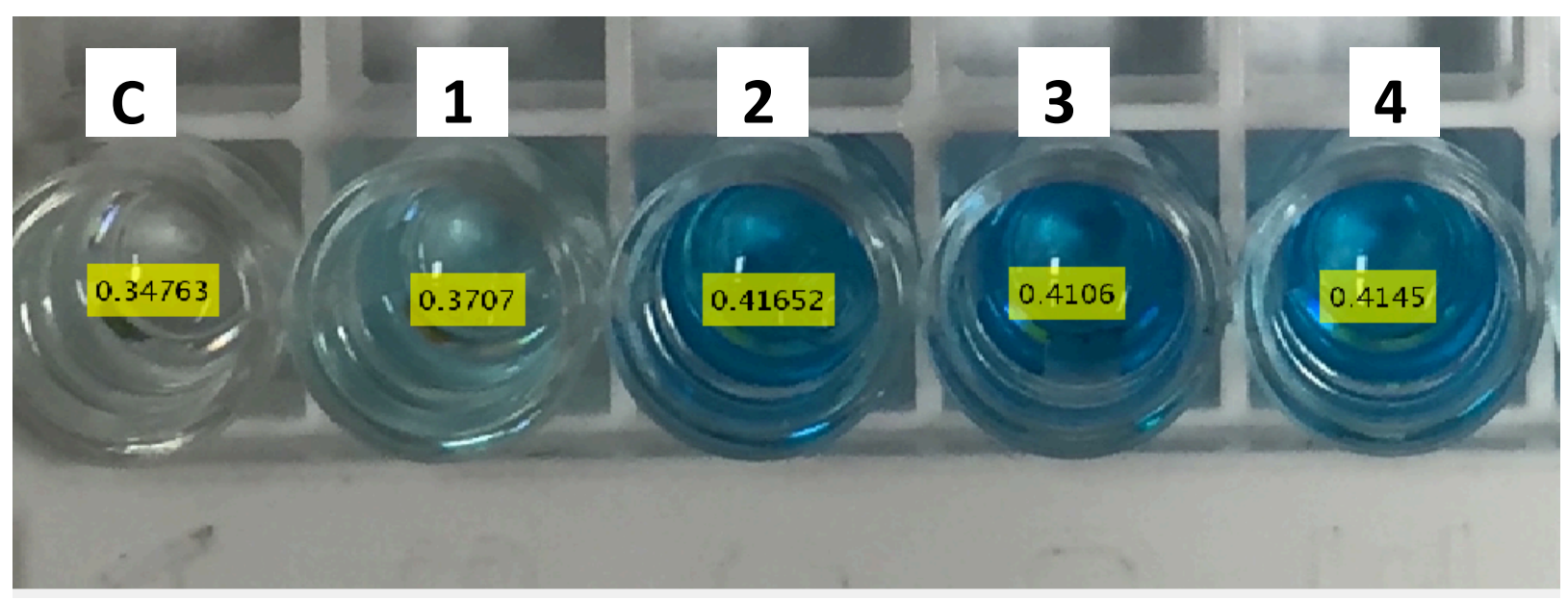

Figure 39: Quantitative Results for the Standard ELISA Test Obtained Using Custom Matlab Code. Well C Used as a Control, Well 1 Using 5 ng Troponin, Well 2 Using 50 ng Troponin, Well 3 Using 500 ng Troponin, and Well 4 Using $1.5 \mu \mathrm{g}$ Troponin.

The troponin masses used in this test were $0 \mathrm{ng}$ (well C), $5 \mathrm{ng}$ (well 1), $50 \mathrm{ng}$ (well 2), $500 \mathrm{ng}$ (well 3), and $1.5 \mu \mathrm{g}$ (well 4). A custom Matlab code was used to quantitatively analyze the results from this standard ELISA test [36]. A ladder effect was seen in the first three wells, from the negative control to channel 2. However, the signal seemed to max out after channel 2 since channels 2,3 , and 4 all had almost identical color intensity values $(\sim 0.41)$. This made it difficult to differentiate between troponin amounts greater than $50 \mathrm{ng}$. 


\section{DISCUSSION}

\section{Dye Testing}

\section{Fluid Connectivity}

As seen in Chapter III, the first circular chip was unable to successfully route fluid throughout the appropriate channels in the dye testing experiments. The first experiment involved the first circular chip within its housing, which was tightened by screws. As seen in Figure 22, the blue fluid used in this test immediately leaked over the channel boundaries and spread throughout the chip in all directions. Since the chip was unable to keep the fluid within the appropriate channels, it was assumed that either the wax borders were printed unsuccessfully or there was not enough pressure applied to keep the fluid from running over the borders randomly. To determine if more pressure would help improve the chip's fluid routing abilities, the next experiment used paper clamps to tighten the housing instead of screws. However, even with these stronger clamps, the chip and housing pair was still unable to successfully route fluid throughout the appropriate channels. As seen in Figure 23, some of the channels were able to contain the fluid in their wax boundaries, but the majority were still unable to. Due to these results, it appeared that pressure was not the only issue.

Since the channels appeared to be too small to properly transport fluid without it spilling over into the wax regions, the next chip was designed to have larger channels. Also, after noticing that the colored wax did not seem to melt all the way through the cellulose paper, the new chip was printed using only black wax. A new housing was also designed to apply more pressure to the chip. A thicker piece of acrylic was used for the bottom housing component to increase pressure when the top housing was screwed into it. After all of these changes were made, the next dye experiment was performed and was a success. The fluid was wicked 
throughout the chip in the appropriate channels with little to no leakage, as seen in Figure 24. From these dye experiments, it was concluded that when the channels on the chip are too small the fluid tends to leak over the boundaries. This is most likely due to the fact that the fluid is wicked at a slow rate through narrow channels so it starts to bead up to avoid the surrounding wax regions. As this fluid build up increases in size, there is a larger force of gravity pulling the bead of water apart and eventually the cohesion forces holding it together are overpowered. This causes the fluid to leak over into the wax regions before being wicked throughout the rest of the channel. Thus, if a chip is unable to route fluid through the appropriate channels, the channel sizes may need to be increased. Also, the black wax was melted through the chip more successfully than the colored waxes so it should always be used when printing the chips. Lastly, to apply more pressure to the chip, a thicker housing should be used. Overall, these initial dye experiments were a crucial part of the design process for both the final chip and housing.

\section{Conjugate Pad}

There were two different conjugate pad materials investigated in this set of dye experiments: cellulose paper and fiberglass strips. Both materials were able to successfully store the red dye solution and then resolubilize it as the sample was wicked through. As seen in Figure 25, the red dye was released from the cellulose conjugate pad and wicked throughout the rest of the chip in the appropriate channels. Similar results were seen for the fiberglass conjugate pad in Figure 26. However, since the fiberglass conjugate pad was cut out and glued to the bottom side of the top layer, it needed to be large enough for the four corners to be glued without interfering with fluid flow. This required the square to be larger than the channel area it covered, providing extra space for fluid to flow. Since the fiberglass was rather absorbent, some of the fluid passing through it traveled horizontally throughout the pad instead of vertically through it. 
This may have led to a loss of signal since the fluid is able to flow to the borders of the conjugate pad instead of through the remainder of the channel beneath the pad. On the other hand, the cellulose paper allows for the dye solution to be dried onto an area surrounded by wax that is equal to the channel size. This ensures that the fluid will flow through the conjugate pad and onto the next layer with little loss. For this reason, the cellulose paper was chosen as the material for the conjugate pad.

Nitrocellulose Detection Layer

Dye testing performed with the nitrocellulose layer was also successful. However, attaching the nitrocellulose layer to the top layer was a bit more challenging. The layer was first attached using super glue. The glue was applied to the designated glue areas on the nitrocellulose layer (all white regions beside the outer ring of circles) and the layer was pressed onto the bottom side of the top layer. This process quickly became messy and the glue was sometimes smudged over the channel areas on the chip. To avoid interfering with fluid flow, tape was used to attach the nitrocellulose layer instead of glue. This method was a lot cleaner and made it easier to line up the channels on the top layer and nitrocellulose layer. Once the nitrocellulose layer was integrated into the 3D chip, the dye testing was performed. As seen in Figure 27, the dye solution was successfully wicked throughout the chip in the appropriate channels.

\section{Development Time Testing}

Based on the results from the development time testing, it was concluded that the 3D microfluidic chip is able to quickly wick the samples throughout its layers, but with much variability. Although the average development times were relatively low, the standard deviations for these times were high. As seen in Table 2, the averages for the three development times 
(time to detection region, time to absorbent pad, and time to absorbent pad for the wash) were all under 4 minutes. These fast development times are desired since the diagnosis of stroke is a time sensitive process. However, all three standard deviations were high relative to each mean. For example, the mean development time to the absorbent pad was 3 minutes and 39 seconds with a standard deviation of 2 minutes and 4 seconds. This large deviation is likely due to the fact that some channels are lined up between layers better than others. Since there are 7 channels per chip it was hard to align the layers so that all 7 channels were perfectly lined up. The channels that were lined up better likely had faster development times since the fluid is able to easily wick from layer to layer. Channels that were misaligned likely had slower development times because there were smaller areas of contact between the channels on different layers, making it harder for the fluid to flow through. This explains why the development times to the absorbent pad ranged from 1 minute and 37 seconds to 7 minutes and 25 seconds. Fortunately, taking an average of these development times helps to correct for these differences. Overall, the chip was efficient in wicking the fluid throughout its layers as long as it was properly aligned.

\section{Antibody Experiments: 3D Paper Microfluidic Device}

\section{$\underline{\text { Initial ELISA Test }}$}

Although GFAP was the intended protein for this project, the biology team ran into complications when trying to pair the protein with an appropriate antibody. Due to the unsuccessful pairing of GFAP with its antibodies, natural human cardiac troponin T protein was used instead. There has been research that suggests the troponin protein is elevated in stroke patients. A study conducted by Mochmann, et al. showed that stroke patients had elevated troponin levels of $>50 \mathrm{ng} / \mathrm{L}$ [35]. Assuming that $10 \mathrm{ml}$ are taken from a patient during a blood draw, any troponin mass of $0.5 \mathrm{ng}$ or more may indicate that the patient has had a stroke. For the 
purposes of this thesis, three different troponin masses were used to see a ladder effect in the signal.

The first ELISA test was unsuccessful in detecting the three masses of troponin in the samples. As seen in Figure 29, the first chip had about the same color intensity values $(\sim 0.33)$ for all seven channels, including the negative control. Similar results were seen for the second chip in Figure 30, with all channels having color intensity values of about 0.34 . Unfortunately, since most of the steps performed during the ELISA cannot be visibly seen, there was no way of knowing what went wrong during the test. Based on issues seen in previous theses projects, the first suspect of error was the conjugate pad. However, since new reagents specific to this project were being used, there is always the question of whether or not the correct concentrations were being used.

To verify that a signal could be produced with the troponin masses used in this first test, a standard ELISA was performed by Jason Dierkhising, a Cal Poly biology student. The same masses of troponin were used in this test: $1 \mathrm{ng}, 10 \mathrm{ng}$, and $100 \mathrm{ng}$. After running the standard ELISA, it was confirmed that these troponin masses were able to produce a signal. This indicated that the signal was either lost on the microfluidic chip or that something was interfering with it. In case of signal loss, higher masses of troponin were used for all future testing: $10 \mathrm{ng}$, $100 \mathrm{ng}$, and $1 \mu \mathrm{g}$. Overall, this initial test was a failure and individual components of the chip/steps in the ELISA process needed to be tested to pinpoint where the test went wrong.

\section{Troubleshooting Experiments}

Detection Antibody Storage on Conjugate Pad

This experiment successfully proved that the detection antibody can be dried and stored on the conjugate pad. As seen in Figure 32, there was an obvious color change on both chips 
used in this experiment, indicating that the antibody was present on the dried conjugate pads. However, the difference in signal seen between the two chips suggests that the material the chips were sitting on top of throughout the storing process affected the results. The pad sitting atop wax-patterned cellulose paper showed a stronger signal than the pad sitting atop regular paper. The green color represents a stronger signal because it indicates that there are traces of yellow mixed into the normal blue signal. The yellow color is a breakdown product from the reaction between HRP and TMB so its presence indicates that more HRP is present and able to react with TMB. Since the wax-backed chip appeared to have more HRP present due to its green signal, it was assumed that more of the detection antibody was successfully stored onto it than the paperbacked chip. This result was expected since the chip sitting atop wax-patterned paper had a hydrophobic barrier preventing the sample from leaving the conjugate pad. However, the chip sitting atop regular paper had nothing to prevent the sample from flowing off the conjugate pad and onto the paper beneath it. This flow from the conjugate pad onto the paper below led to a loss of signal, as seen by the color difference between the two chips. Overall, it was concluded that all chips should be prepared and dried atop wax paper to maximize the storage of antibodies and signal.

\section{Inclusion vs. Exclusion of Conjugate Pad}

Since the conjugate pad has caused issues in previous theses experiments, it was the main focus of concern for this project. This test allowed for a direct comparison between a chip that included the conjugate pad and a chip that excluded the conjugate pad. As seen in Figure 32, the two channels that excluded the conjugate pad from their chip arrangement showed a color change, while the two channels that included the conjugate pad did not. Equal masses of troponin (100 $\mathrm{ng}$ in one channel and $1 \mu \mathrm{g}$ in the other) were used for both sets of channels so in 
theory the signals should have been identical between the two sets. However, the channels without the conjugate pad present had a positive signal while the channels with the conjugate pad had a negative signal. Based on these results, it was concluded that the conjugate pad was interfering with the signal on the chip. Since previous experiments have proven that the conjugate pad is able to successfully store the detection antibody, it can be assumed that the problem lies within the resolubilization step. Even though the antibody is stored on the conjugate pad, the lack of signal indicates that it is unable to make it to the detection region. Thus, it is likely that the detection antibody never left the conjugate pad. This suggests that the sample is unable to successfully resolubilize the detection antibody as it passes through the conjugate pad. However, further experiments would need to be performed to determine if this is actually the cause of the lack of signal. For the purposes of this thesis, the conjugate pad was excluded in all future tests to ensure a better signal.

\section{BSA Blocking vs. No Blocking}

Due to the lack of signal seen in the first ELISA experiment, it was questioned whether or not the BSA blocking step was interfering with the binding of troponin to the detection and capture antibodies. To determine the affect of BSA on the ELISA signal, another test was run to compare two channels that were BSA blocked with two channels that were not blocked at all. As seen in Figure 36, there were distinct differences between the two channel sets. The channels that were blocked with BSA (1 and 2) were both a blue color with color intensity values of about 0.39. On the other hand, the unblocked channels ( 3 and 4) were both a blue color at first, but then channel 4 quickly turned green and then yellow. As mentioned before, this yellow color is a breakdown product of the reaction between TMB and HRP and indicates that more HRP is

present than in the other channels. However, since the Matlab code used to process the images is 
designed to analyze a blue color, it is unable to give an accurate reading for the yellow color. Thus, it assigned channel 4 a lower color intensity value (0.37) than the other channels even though the yellow color actually indicates a stronger signal.

In conclusion, the unblocked channels showed a stronger signal than the BSA blocked channels for the same mass of troponin. However, since there was still a strong signal seen in the BSA blocked channels it was concluded that the BSA was not interfering with the interaction between troponin and the detection and capture antibodies. Based on these results, it was assumed that the stronger signal seen in the unblocked channels was due to non-specific binding of the HRP detection antibody to the nitrocellulose layer. This was likely causing a false amplification of the signal. To prevent this non-specific binding from contributing to the signal, all future tests blocked the nitrocellulose layer with BSA before use.

\section{$\underline{\text { Final ELISA Test }}$}

After fine-tuning each step in the ELISA process, the final test was run successfully. However, human error was still a factor in this test as the the results were not entirely perfect. As seen in Figure 38, the ladder effect in the signal for the three masses of troponin (10 ng, 100 $\mathrm{ng}$, and $1 \mu \mathrm{g}$ ) was successfully portrayed in one channel set, but not the other. Channels 4 through 6 showed an incremental increase in the color intensity values (from 0.349 to 0.369 ). Channels 1 through 3, on the other hand, showed an increase in color intensity values between channels 1 and 2 , but not 2 and 3 . This indicated a loss of signal in channel 3, likely due to human error throughout the experiment. Another explanation for this may be that since the surface area of the detection region is relatively small, the area may have become saturated with the troponin solution at the lower mass, making the signal plateau at that lower level. This would explain why each of the three masses had very similar signal intensity values. However, 
all six of the channels showed a positive signal and the ladder effect was seen in both channel sets for the most part. Based on these results, it was concluded that biologically relevant masses of troponin can be successfully detected on the microfluidic device.

One inconsistency noted was the variability in the color intensity values between channels with equal troponin masses. Compared to the BSA blocked channels in the BSA Blocking vs. No Blocking experiment, the channels in the final ELISA test had substantially lower color intensity values even though they were exposed to equal troponin masses. This may have been due to the fact that the troponin/antibody solution in the BSA experiment was introduced directly to the detection region on the chip, while the troponin/antibody solution in the final ELISA experiment was introduced to the sample pad. From the sample pad, the solution had to travel down the channel to reach the detection region. While traveling from the sample pad, some of the troponin/antibody in the sample may have been lost along the way before reaching the detection region. This would cause a loss in signal and would explain why the final ELISA test had lower color intensity values than the previous BSA experiment. However, overall this final test was successful in detecting the desired troponin masses.

To compare the paper-based ELISA to a standard ELISA, similar troponin masses were used in a standard ELISA performed in wells. This test was performed by Jason Dierkhising, a biology student at Cal Poly San Luis Obispo. Although the masses were not identical to the ones used in this thesis, a comparison was still able to be made. As seen in Figures 38 and 39, the color intensity values for the negative controls in both the paper-based and standard ELISA tests were similar ( 0.34). This suggested that the Matlab code was successful in correcting for variable lighting conditions since it was able to process two pictures taken from different cameras in different lighting and assign almost identical color intensity values to the control 
channels. Since the $5 \mathrm{ng}$ sample used in the standard ELISA test was the closest to the $10 \mathrm{ng}$ sample used in the final paper-based ELISA test, a comparison was made between their color intensity values. The $5 \mathrm{ng}$ well in the standard ELISA had an intensity value of 0.37 , while the $10 \mathrm{ng}$ channels in the final ELISA test had an average value of about 0.35 . The paper-based ELISA had a higher amount of troponin and yet a lower color intensity value, suggesting that the paper platform experienced signal loss. Furthermore, both the $100 \mathrm{ng}$ and $1 \mu \mathrm{g}$ channels on the paper-based ELISA had lower color intensity values than the $5 \mathrm{ng}$ well in the standard ELISA. The weaker signals produced in the paper-based ELISA are likely due to a loss of reagents in the chip as the sample flows throughout the channels. Unlike the standard ELISA, where all of the reagents are contained in a single well, the ELISA performed on the microfluidic device is spread throughout the entire chip. Some of the protein/antibodies are likely to be left behind as the sample flows down the channel. Since the signal is read in only one place on the chip (detection region) these proteins/antibodies that were left behind in other regions are unable to contribute to the signal. This leads to signal loss and thus explains why the paper-based ELISA had weaker signals overall.

Although the final ELISA test performed on the paper microfluidic device showed weaker signals than the standard ELISA, it was still enough to indicate a positive signal and show a ladder effect between different troponin masses. This thesis successfully proved that ELISA techniques can be incorporated onto a paper microfluidic device. The signal loss seen on this paper platform can be further investigated in future experiments. 


\section{CONCLUSIONS}

Overall, this thesis demonstrated that a 3D paper-based microfluidic device is able to successfully detect biologically relevant concentrations of a stroke biomarker utilizing ELISA techniques. The microfluidic chip was fabricated out of cellulose and nitrocellulose membranes and was able to successfully route fluid through designated channels. An external housing was made to apply pressure to the chip and ensure connectivity between layers. This connectivity was confirmed through dye experiments, verifying that the external housing applied adequate pressure to the microfluidic chip to allow for both horizontal and vertical fluid flow. Furthermore, ELISA techniques were successfully integrated into the microfluidic chip, as shown by the generation of a signal on the device after the introduction of TMB substrate. This signal indicated that the troponin protein was able to be sandwiched between the capture and detection antibody, allowing for the HRP-conjugated detection antibody to react with the TMB substrate and generate a blue signal. This was a major accomplishment of the project because the paper-based microfluidic chip was able to accommodate for the wash steps required in an ELISA. This was an important success because most current paper-based chips have not had the ability to allow for wash steps. With the circular chip design and its ability to spin to open up new channels, the wash steps were successfully performed in the ELISA experiments.

Furthermore, incorporation of ELISA techniques into the microfluidic chip, allowed for a quantitative analysis of the results. Using custom Matlab code [36], color intensity values were assigned to each detection region. Color intensity values have a linear correlation with the amount of troponin. This automated visual analysis allows for not only the detection of the stroke biomarker, but also the calculation of its concentration. Using this technology, doctors across the world can accurately differentiate between the two types of stroke in a more time 
efficient manner. Since many stroke biomarkers are normally present in the human body, strokes are often correlated with a change in the concentration of these biomarkers. Thus, without this quantitative analysis it is impossible to diagnose stroke using these biomarkers since detection is not sufficient to accurately diagnose stroke. The integration of ELISA technology and paper microfluidic techniques is a major step toward improving the stroke diagnostic process because it allows for the use of stroke biomarkers in a rapid, cost-effective technology. This thesis showed that a paper microfluidic device can successfully detect a stroke biomarker and display a signal with a color intensity that correlates with its concentration. This simpler diagnostic process will allow for a quicker and more appropriate treatment of stroke patients and provide significant clinical impact by saving lives. 


\section{FUTURE WORK}

Although this project was successful, there are aspects of the system that could be improved for better results. For example, the alignment of the chip layers proved to be difficult during the dye experiments. Since each chip had seven channels, it was challenging to align all the channels simultaneously during assembling. Furthermore, keeping the chip aligned while being placed into the housing was even more of a challenge since the layers would move relative to one another during the process. After the chip was assembled, positioned in the housing, and secured by tightening the housing, the layers were almost always shifted slightly. These small misalignments were detected during the dye experiments when the fluid was unable to successfully flow throughout the channel at a reasonable rate. This misalignment also affected the development time results, causing some channels to have slow development times due to the poor connection between layers. To address this issue, a new housing component could be developed that better aligns and secures the chip. For instance, the bottom housing piece could have small pegs sticking up around the outside border. The chips could then have small holes cut out in the appropriate locations so that they could be inserted onto the pegs individually. This would help secure the chips as they are being assembled. Furthermore, the pegs would also insert through the top housing piece, allowing the housing to be tightened without having to move the chip. This would allow for an easier and more accurate assembly of the chip within the housing, which would produce more consistent results for both the dye and development time testing.

Another component that could be improved in the future is the conjugate pad. Since the conjugate pad seemed to interfere with the signal in this project, a set of experiments could help determine the root causes of the issues with the pad. Based on the results from this thesis, it is 
assumed that the conjugate pad is unable to properly resolubilize the detection antibody after it is dried. A number of solutions could be used to help with this resolubilization process, including the use of ionic detergents such as SDS. However, these detergents are known to be harsh on proteins and thus not desirable since they run the risk of denaturing the proteins and interfering with the signal. Additionally, the sample could soak on top of the conjugate pad for an extended period of time before allowing it to flow throughout the rest of the chip. This pre-soaking of the pad could be achieved by adding and additional spin position to the chip design. The first chip position would have the channels on the top layer and conjugate pad skewed to the left of the channels on the rest of the layers. This design would put the sample and conjugate pad above wax regions on the remaining layers. When the sample is first introduced to the sample pad, it will flow through to the conjugate pad and then stop due to a wax barrier beneath the conjugate pad, allowing the sample to soak on the conjugate pad for however long is needed to resolubilize the detection antibody. After a specified time, the top two layers would be spun to align their channels with the channels on the layers beneath them, allowing for the sample to continue to flow throughout the rest of the chip. The ELISA would then be performed normally from this step on. This new design could improve resolubilization of the detection antibody from the conjugate pad by allowing for longer contact time between the sample and the dried antibody.

Future work could also focus on reducing the signal loss observed with the paper-based ELISA. For instance, the microfluidic chip could be altered to prevent any sample from being left on the paper. Different wash solutions could be compared to determine if they improve efficiency in picking up lost reagents and delivering them to the detection region, thus increasing the signal. Furthermore, the channel lengths could be reduced to determine if a shorter travel distance minimizes the amount of reagents lost. Ideally, the channels would all be stacked one 
on top of the other in a single circular region, mimicking the idea of wells on a paper platform. This design would need to be tested to ensure that there is still enough channel length for the sample to flow through each region in an adequate time frame for protein-antibody binding to occur. Also, the design would need to prevent cross-over contamination between regions, which could lead to a false positive signal. For example, if any detection antibody remains on the conjugate pad, which would sit atop the detection region, then it may react with the TMB substrate once it is added, generating a false positive signal.

Lastly, future experiments could be run to investigate if the small surface area of the detection region was responsible for the little change in signal intensity seen between the three troponin masses. There is reason to believe the detection region was too small to accommodate the larger troponin masses, causing the detection region to become saturated at a lower concentration. To further investigate this speculation, testing could be performed using various sized diameters for the detection region. The same troponin mass would be used on all of the different sized diameters in order to see if there was a difference in the signal. If the larger diameters show a stronger signal, there is evidence to suggest the smaller diameters became saturated and were unable to show the full signal due to lack of available binding sites for the capture antibodies.

Overall, this thesis proved that an ELISA can be performed on a paper microfluidic device, but there are still many aspects of this process that could use improvement. Hopefully, through future work performed in this area of research, a paper-based ELISA will produce a signal that is as strong and as accurate as that of a standard ELISA. 


\section{REFERENCES}

[1] "Stroke." World Heart Federation. World Heart Federation, n.d. Web. 06 Nov. 2016. $<$ http://www.world-heart-federation.org/cardiovascular-health/stroke/>.

[2] "Nine Percent to 30\% of Strokes Misdiagnosed in ED." AHC Media Continuing Medical Education Publishing RSS. AHC Media, 1 July 2013. Web. 06 Nov. 2016. $<$ https://www.ahcmedia.com/articles/63612-nine-percent-to-30-of-strokes-misdiagnosedin-ed $>$.

[3] Saenger, A. K., and R. H. Christenson. "Stroke Biomarkers: Progress and Challenges for Diagnosis, Prognosis, Differentiation, and Treatment." Clinical Chemistry 56.1 (2009): 21-33. Web. 15 Sept. 2015.

[4] "Types of Stroke - NHLBI, NIH." U.S National Library of Medicine. U.S. National Library of Medicine, 22 June 2016. Web. 06 Nov. 2016. $<$ https://www.nhlbi.nih.gov/health/health-topics/topics/stroke/types>.

[5] "Stroke Research | Neuroscience Research | Tocris Bioscience." Tocris. Tocris Bioscience, n.d. Web. 06 Nov. 2016. $<$ https://www.tocris.com/researchArea.php?ItemId=128726\#.V6SSt_krIqN $>$.

[6] "Stroke Treatments." Stroke Association. American Heart Association, n.d. Web. 06 Nov. 2016. <http://www.strokeassociation.org/STROKEORG/AboutStroke/Treatment/StrokeTreatments_UCM_310892_Article.jsp\#.WARNEZMrKRs>.

[7] "CT Scan vs MRI." Diffen. Diffen, n.d. Web. 06 Nov. 2016. $<$ http://www.diffen.com/difference/CT_Scan_vs_MRI $>$.

[8] "Types of Stroke." Stroke Association. American Heart Association, n.d. Web. 06 Nov. 2016. 
$<$ http://www.strokeassociation.org/STROKEORG/AboutStroke/TypesofStroke/Types-ofStroke_UCM_308531_SubHomePage.jsp>.

[9] "Magnetic Resonance Angiography (MRA)." Hopkins Medicine. The John Hopkins University, n.d. Web. 06 Nov. 2016.

$<$ http://www.hopkinsmedicine.org/healthlibrary/test_procedures/cardiovascular/magnetic _resonance_angiography_135,14/>.

[10] "CT Scan." Stroke Center. The Internet Stroke Center, n.d. Web. 06 Nov. 2016. $<$ http://www.strokecenter.org/patients/stroke-diagnosis/imaging-tests/ct-scan/>.

[11] Gubala, Vladimir, Leanne F. Harris, Antonio J. Ricco, Ming X. Tan, and David E. Williams. "Point of Care Diagnostics: Status and Future." Analytical Chemistry 84 (2012): 487-515. ACS Publications. American Chemistry Society. Web. 25 Aug. 2016.

[12] Sia, Samuel K., and Larry J. Kricka. "Microfluidics and Point-of-care Testing." Lab on a Chip 8.12 (2008): 1982. The Royal Society of Chemistry. Web. 16 June 2016.

[13] Martinez, Andres W., Scott T. Phillips, and George M. Whitesides. "Diagnostics for the Developing World: Microfluidic Paper-Based Analytical Devices." Analytical Chemistry 82 (2010): 3-10. ACS Publications. Analytical Chemistry, 1 Jan. 2010. Web. 12 July 2016. <http://pubs.acs.org/doi/pdf/10.1021/ac9013989>.

[14] Millipore Corporation. Rapid Lateral Flow Test Strips: Considerations for Product Development. Billerica: Millipore Corporation, 2008. Print.

[15] "Frequently Asked Questions (FAQ's)." MDI Membrane. Advanced Microdevices, n.d. Web. 06 Nov. 2016. <http://www.mdimembrane.com/FAQs.html>.

[16] Zhao, Limian, and Phu Duong. Choosing the Best Syringe Filters for Biological Sample Filtration. Rep. N.p.: Agilent Technologies, 2012. Print. 
[17] Man, Protein. "Which Blocking Agent for Western Blotting?" G-Biosciences, 28 Sept. 2012. Web. 06 Nov. 2016. <http://info.gbiosciences.com/blog/bid/159273/WhichBlocking-Agent-for-Western-Blotting>.

[18] "Enhancement of the Detection Limit for Lateral Flow Immunoassays: Evaluation and Comparison of Bioconjugates." National Center for Biotechnology Information. U.S. National Library of Medicine, 31 Jan. 2012. Web. 06 Nov. 2016. $<$ http://www.ncbi.nlm.nih.gov/pubmed/22100215>.

[19] Ward, Jennifer. Nitrocellulose Paper Based Microfluidic Platform Development and Surface Functionalization with Anit-IgE Aptamers. Thesis. Cal Poly San Luis Obispo, 2012. San Luis Obispo: Cal Poly San Luis Obispo, 2012. Print.

[20] "Cellulose Acetate Membrane Filters." Advantec MFS, Inc., n.d. Web. 06 Nov. 2016. $<$ http://www.advantecmfs.com/filtration/membranes/mb_ca.shtml $>$.

[21] "Lateral Flow Immunoassays." Cytodiagnostics, Inc., n.d. Web. 06 Nov. 2016. $<$ http://www.cytodiagnostics.com/store/pc/Lateral-Flow-Immunoassays-d6.htm>.

[22] Lu, Yao, Welwel Shi, Jianhua Qin, and Bingcheng Lin. "Fabrication and Characterization of Paper-Based Microfluidics Prepared in Nitrocellulose Membrane by Wax Printing." Analytical Chemistry 82 (2010): 329-35. Web. 18 Sept. 2016.

[23] NASA. "Reynolds Number." Glenn Research Center, n.d. Web. 06 Nov. 2016. $<$ https://www.grc.nasa.gov/www/BGH/reynolds.html $>$.

[24] "Reynolds Number." The Engineering Toolbox. N.p., n.d. Web. 06 Nov. 2016. $<$ http://www.engineeringtoolbox.com/reynolds-number-d_237.html $>$.

[25] Ducree, Jens. Physics of Microfluidic Systems. N.d. Lecture slides. $<$ http://dcu.ie/ ducreeje/myfluidix/Materials/03_Physics_01.pdf $>$. 
[26] Bruus, Henrik. "Chapter 1 Governing Equations in Microfluidics." Royal Society of Chemistry, 2014. Web. 06 Nov. 2016.

$<$ http://pubs.rsc.org/en/content/chapterhtml/2014/bk9781849736718-00001?isbn=978-184973-671-8>.

[27] Berthier, Jean, Kenneth A. Brakke, and Erwin Berthier. "Chapter 7: Paper-Based Microfluidics." Open Microfluidics. Hoboken, NJ: John Wiley \& Sons, 2016. 229-57. Print.

[28] Or, Dani, and Markus Tuller. Capillarity. N.p., 13 Sept. 2013. Web. 7 Nov. 2016. $<\mathrm{http}: / /$ www.engr.uconn.edu/environ/envphys/pdf/vadose_pdf/Capillarity_revised01.pd $>$.

[29] "Chapter 2: ELISA Formats." Bio-Rad, n.d. Web. 06 Nov. 2016. <https://www.bio-radantibodies.com/elisa-types-direct-indirect-sandwich-competition-elisa-formats.html>.

[30] R\&D Systems. ELISA Development Guide. N.p.: R\&D Systems, n.d. Print.

[31] Reynolds, M. A. "Early Biomarkers of Stroke." Clinical Chemistry 49.10 (2003): 1733-739. Web.

[32] Ramos-Fernandez, M., M. F. Bellolio, and L. G. Stead. "Matrix Metalloproteinase-9 as a Marker for Acute Ischemic Stroke: A Systematic Review." National Center for Biotechnology Information. U.S. National Library of Medicine, 17 June 2010. Web. 06 Nov. 2016. <http://www.ncbi.nlm.nih.gov/pubmed/21044610>.

[33] Foerch, C., I. Curdt, B. Yan, F. Dvorak, M. Hermans, J. Berkefeld, A. Raabe, T. NeumannHaefelin, H. Steinmetz, and M. Sitzer. "Serum Glial Fibrillary Acidic Protein as a Biomarker for Intracerebral Haemorrhage in Patients with Acute Stroke." Journal of Neurology, Neurosurgery \& Psychiatry 77.2 (2006): 181-84. Web.

[34] Tageson, Mackenzie. Functional 3D Cellulose and Nitrocellulose Paper-based, Multiplex 
Diagnostic Platforms Without Coupling Agents. Thesis. Cal Poly San Luis Obispo, 2013. San Luis Obispo: Cal Poly San Luis Obispo, 2013. Print.

[35] Mochmann, H. C., J. F. Scheitz, G. C. Petzoid, K. G. Haeusler, H. J. Audebert, U. Laufs, C. Schneider, U. Landmesser, N. Werner, M. Endres, B. Witzenbichler, C. H. Nolte, and TRELAS Study Group. "Coronary Angiographic Findings in Acute Ischemic Stroke Patients With Elevated Cardiac Troponin: The Troponin Elevation in Acute Ischemic Stroke (T..." National Center for Biotechnology Information. U.S. National Library of Medicine, 1 Mar. 2016. Web. 06 Nov. 2016.

$<$ https://www.ncbi.nlm.nih.gov/pubmed/26933082?dopt=Abstract $>$.

[36] Huynh, Erik V. Development of a 3-D Microfluidic Paper-based Analytical Device for ELISA Reagents and a Smartphone Image Analysis Diagnostic Algorithm. Cal Poly San Luis Obispo, 16 June 2016. Web. 25 Sept. 2016.

[37] http://pubs.rsc.org/services/images/RSCpubs.ePlatform.Service.FreeContent.Image Service.svc/ImageService/Articleimage/2013/LC/c3lc50169h/c3lc50169h-f3.gif [38] Sandwich ELISA: https://www.lsbio.com/image2/human-alpha-fetoprotein-elisa-kitsandwich-elisa-ls-f10000/255806_1927627.jpg 


\section{APPENDICES}

\section{APPENDIX A: CHIP AND HOUSING DIMENSIONS}

The following figures show the dimensions of the different chip designs and housing component used in this project. The dimensions are reported in millimeters.

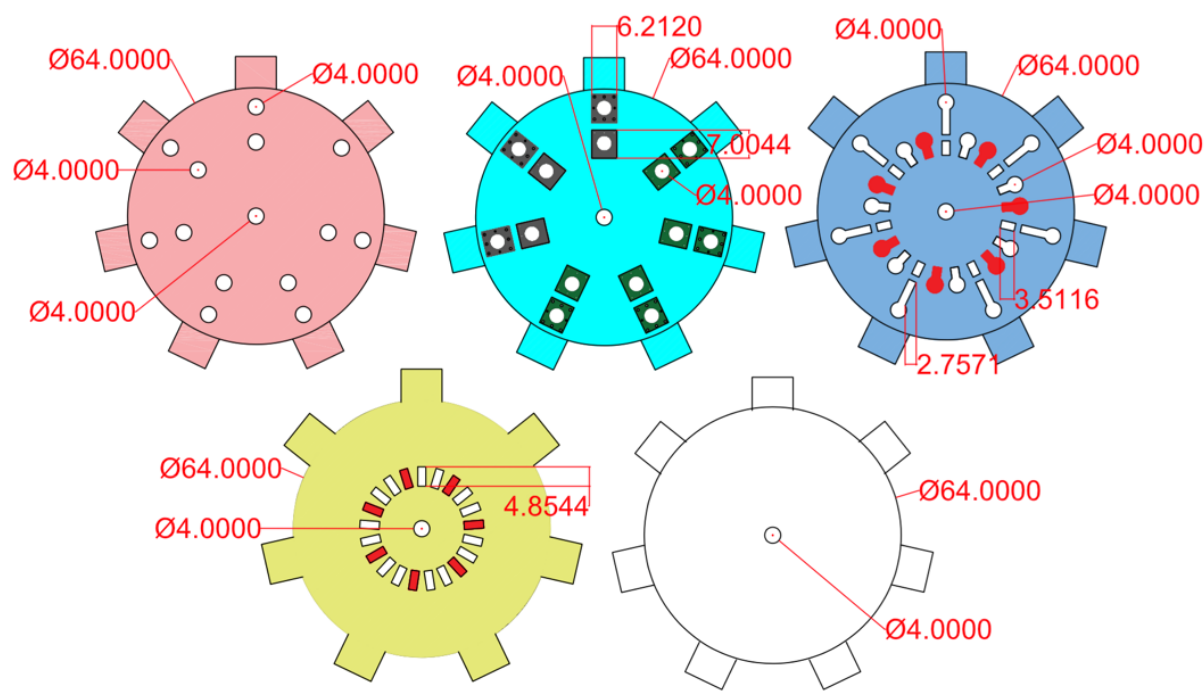

Figure 40: Dimensions of the Second Chip Design (First Circular Chip)

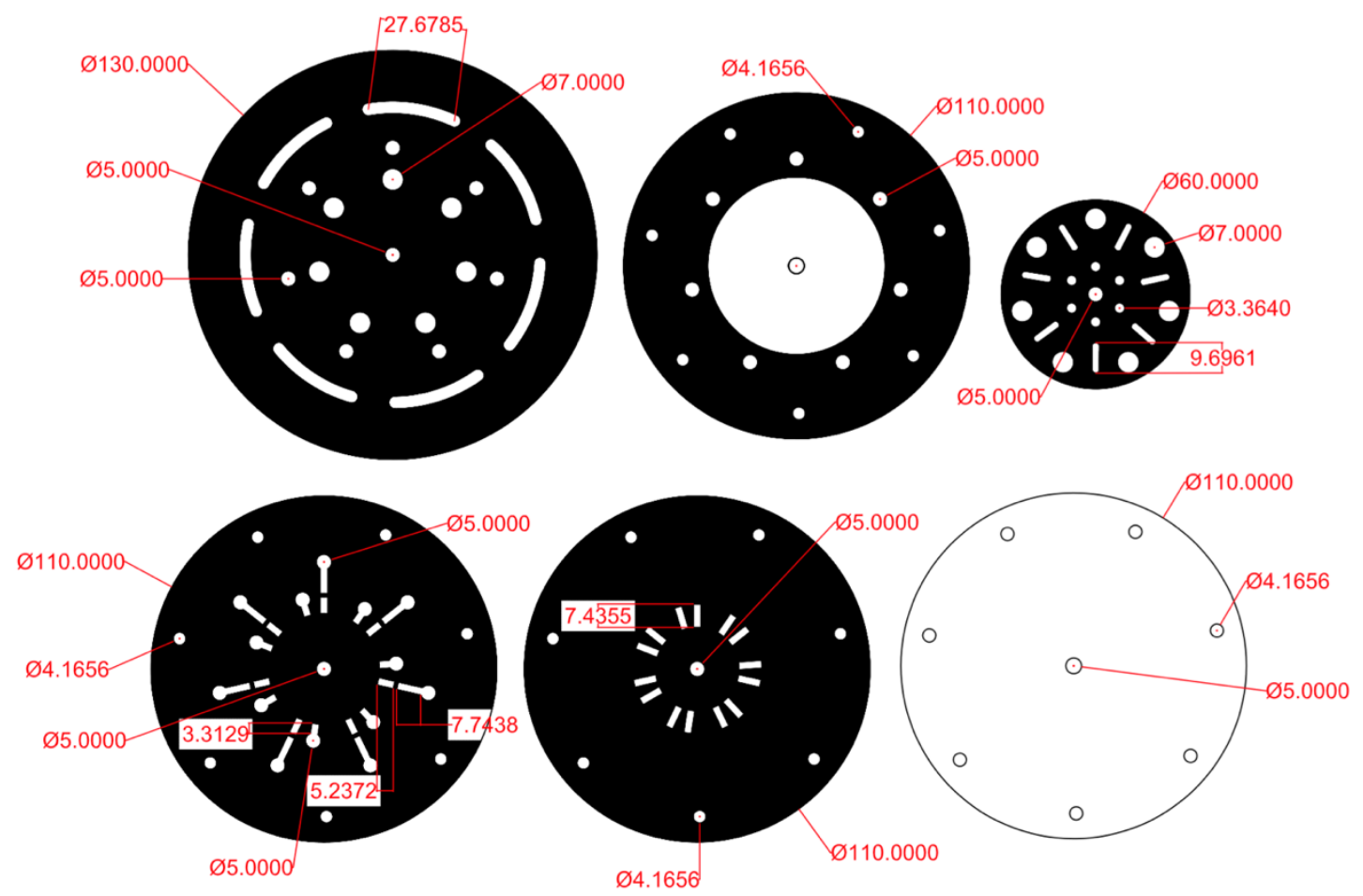

Figure 41: Dimensions of the Final Chip 


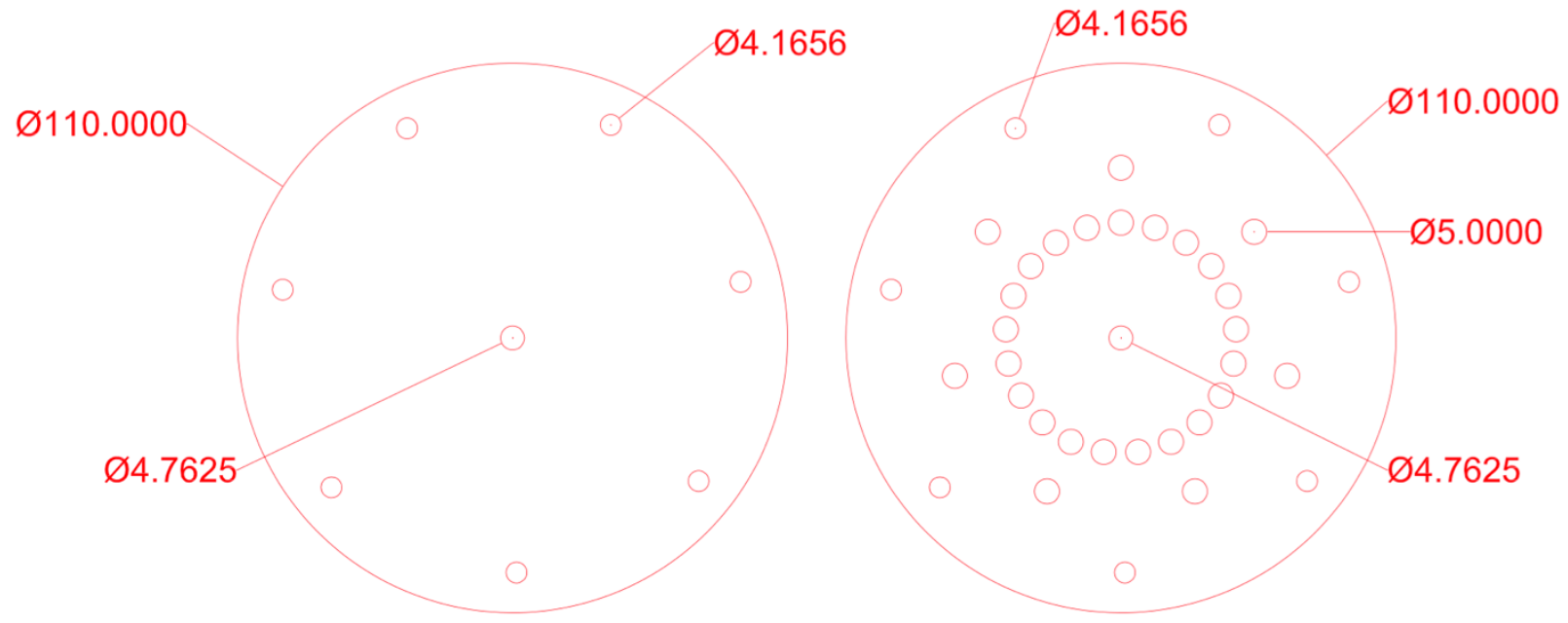

Figure 42: Dimensions of the Initial Housing Components
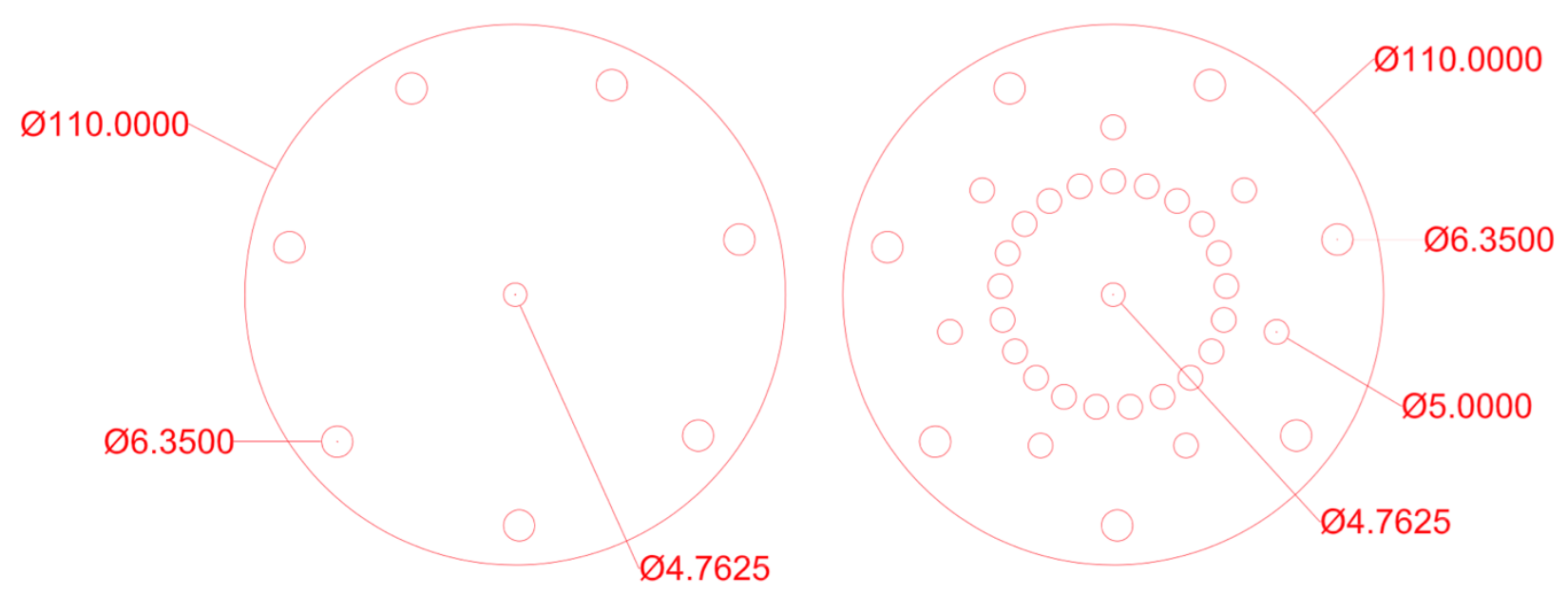

Figure 43: Dimensions of the Final Housing Components 


\section{APPENDIX B: BIOCHEMISTRY CALCULATIONS}

\section{Preparation of the Stock Solutions}

Preparation of the BSA stock solution:

Measure out $80 \mu \mathrm{l}$ of initial BSA solution

$\left(1 \frac{u g}{u l}(B S A\right.$ solution $\left.)\right) * 80 u l$ sample $=80 u g B S A$

Add $720 \mu l$ of DI water

$\left(\frac{80 u g(B S A)}{(720 u l(D I \text { water })+80 u l(B S A)}\right)=0.1 \frac{u g}{u l}=100 \frac{\boldsymbol{u g}}{\boldsymbol{m l}}$ BSA solution

Preparation of the BSA buffer solution:

Dissolve $0.1 \mathrm{~g}$ of sucrose in $500 \mu \mathrm{l}$ of water

$\left(\frac{g \text { (sucrose })}{500 u l(\text { DI water })}\right)=200 \frac{u g}{u l}$ sucrose solution

Measure out $24 \mu \mathrm{l}$ of initial BSA solution

$\left(1 \frac{u g}{u l}(B S A\right.$ solution $\left.)\right) * 24 u l$ sample $=24 u g B S A$

Add $216 \mu 1$ of DI water and $2 \mu 1$ of sucrose solution

$$
\begin{gathered}
\left(\frac{24 u g(B S A)}{(216 u l(D I \text { water })+2 u l(\text { sucrose solution }))}=0.11 \frac{u g}{u l}\right. \\
=\mathbf{1 1 0} \frac{\mathbf{u g}}{\mathbf{m l}} \text { BSA buffer solution }
\end{gathered}
$$

Preparation of the streptavidin stock solution:

Measure out $36 \mu l$ of initial streptavidin solution

$$
\left(0.5 \frac{u g}{u l}(\text { streptavidin solution })\right) * 36 u l \text { sample }=18 \text { ug streptavidin }
$$


Add $240 \mu$ of DI water

$\left(\frac{18 u g(\text { streptavidin })}{(240 u l(D I \quad \text { ater })+36 u l(\text { streptavidin }))}\right)=0.065 \frac{\boldsymbol{u g}}{\boldsymbol{u l}}$ streptavidin solution

\section{Preparation of the Antibody Stock Solutions}

Preparation of the capture antibody stock solution:

Measure out $2 \mu \mathrm{l}$ of initial capture antibody solution

$\left(2 \frac{u g}{u l}(\right.$ capture antibody solution $\left.)\right) * 2$ ul sample $=4$ ug capture antibody

Add $30 \mu 1$ of DI water

$$
\begin{gathered}
\left(\frac{4 u g(\text { capture antibody) }}{(30 \text { ul (DI water) }+2 u l(\text { capture antib dy }))}\right) \\
=0.125 \frac{\boldsymbol{u g}}{\boldsymbol{u l}} \text { capture antibody solution }
\end{gathered}
$$

Preparation of the HRP-conjugated detection antibody stock solution:

Measure out $5 \mu \mathrm{l}$ of initial detection antibody solution

$\left(0.9 \frac{u g}{u l}(\right.$ detection antibody solution $\left.)\right) * 5$ ul sample $=4.5$ ug detection antibody

Add $85 \mu 1$ of DI water

$$
\begin{gathered}
\left(\frac{4.5 \mathrm{ug}(\text { detection antibody })}{(85 u l(\text { DI water })+5 u l(\text { detection antibody }))}\right)=0.05 \frac{u g}{u l} \\
=\mathbf{5 0} \frac{\mathbf{n g}}{\mathbf{u l}} \text { detection antibody solution }
\end{gathered}
$$




\section{Minimum Troponin Mass in Stroke Patients}

$\left(50 \frac{\mathrm{ng}}{\mathrm{L}}(\right.$ serum concentration of troponin in a stroke patient $\left.)\right) *\left(\frac{1 \mathrm{~L}}{1000 \mathrm{ml}}\right)=0.05 \frac{\mathrm{ng}}{\mathrm{ml}}$ $\left(0.05 \frac{\mathrm{ng}}{\mathrm{ml}}(\right.$ troponin solution $\left.)\right) * 10 \mathrm{ml}=\mathbf{0 . 5} \mathbf{n g}$

\section{Preparation of Troponin Solutions}

Preparation of $100 \mathrm{ng}$ troponin solution used in the initial ELISA test:

Measure out $2 \mu \mathrm{l}$ of stock troponin solution

$\left(1.93 \frac{u g}{u l}(\right.$ troponin sol tion $\left.)\right) * 2$ ul sample $=3.86 u g$ troponin

Add $77 \mu 1$ of DI water

$\left(\frac{3.86 u g(\text { troponin })}{(77 u l(\text { DI water })+2 u l(\text { troponin }))}\right)=0.049 \frac{u g}{u l}$ troponin solution

Pipette $2 \mu l$ of this troponin solution onto each detection region

$\left(0.049 \frac{u g}{u l}(\right.$ troponin solution $\left.)\right) * 2$ ul sample $\approx 0.10 u g=100$ ng troponin

Preparation of $10 \mathrm{ng}$ troponin solution used in the initial ELISA test:

Measure out $2 \mu \mathrm{l}$ of the $100 \mathrm{ng}$ troponin solution

$\left(0.049 \frac{u g}{u l}(\right.$ troponin solution $\left.)\right) * 2$ ul sample $\approx 0.10 u g$ troponin

Add $18 \mu$ of DI water

$\left(\frac{0.10 u g(\text { troponin })}{(18 u l(\text { DI water })+2 u l(\text { troponin }))}\right)=0.005 \frac{u g}{u l}$ troponin solution

Pipette $2 \mu \mathrm{l}$ of this troponin solution onto each detection region 


$$
\left(0.005 \frac{u g}{u l}(\text { troponon solution })\right) * 2 u l \text { sample }=0.01 u g=10 \text { ng troponin }
$$

Preparation of $1 \mathrm{ng}$ troponin solution used in the initial ELISA test:

Measure out $2 \mu \mathrm{l}$ of the $100 \mathrm{ng}$ troponin solution

$$
\left(0.049 \frac{u g}{u l}(\text { troponin solution })\right) * 2 \text { ul sample } \approx 0.10 u g \text { troponin }
$$

Add $198 \mu$ l of DI water

$$
\left.\left(\frac{0.10 u g(\text { troponin })}{(198 u l(\text { DI water })+2 u l(\text { tropon }} n\right)\right)=0.0005 \frac{u g}{u l} \text { troponin solution }
$$

Pipette $2 \mu \mathrm{l}$ of this troponin solution onto each detection region

$$
\left(0.0005 \frac{u g}{u l}(\text { troponon solution })\right) * 2 \text { ul sample }=0.001 \mathrm{ug}=\mathbf{1} \mathbf{n g} \text { troponin }
$$

Preparation of $1 \mu \mathrm{g}$ troponin solution used in the final ELISA test:

Measure out $3 \mu \mathrm{l}$ of the stock troponin solution

$\left(1.93 \frac{u g}{u l}\right.$ (troponin solution $\left.)\right) * 3$ ul sample $=5.79$ ug troponin

Add $8.58 \mu 1$ of DI water

$$
\left(\frac{5.79 u g(\text { troponin })}{(8.58 u l(\text { DI water })+3 \text { ul (troponin }))}\right)=0.5 \frac{u g}{u l} \text { troponin solution }
$$

Measure out $10 \mu \mathrm{l}$ of this troponin solution

$$
\left(0.5 \frac{u g}{u l}(\text { troponin solution })\right) * 10 u l \text { sample }=5 \text { ug troponin }
$$

Add $90 \mu \mathrm{l}$ of DI water

$$
\left(\frac{5 u g(\text { troponin })}{(90 u l(\text { DI water })+10 u l(\text { troponin }))}\right)=0.05 \frac{u g}{u l} \text { troponin solution }
$$


Pipette $20 \mu \mathrm{l}$ of this troponin solution onto each detection region

$\left(0.05 \frac{u g}{u l}(\right.$ troponon solu ion $\left.)\right) * 20$ ul sample $=1$ ug troponin

Preparation of $100 \mathrm{ng}$ troponin solution used in the final ELISA test:

Measure out $10 \mu \mathrm{l}$ of the $1 \mu \mathrm{g}$ troponin solution

$\left(0.05 \frac{u g}{u l}(\right.$ troponin solution $\left.)\right) * 10 u l$ sample $=0.5 u g$ troponin

Add $90 \mu l$ of DI water

$\left(\frac{0.5 u g(\text { troponin })}{(90 u l(D I \text { water })+10 u l(\text { troponin }))}\right)=0.005 \frac{u g}{u l}$ troponin solution

Pipette $20 \mu \mathrm{l}$ of this troponin solution onto each detection region

$\left(0.005 \frac{g}{u l}(\right.$ troponon solution $\left.)\right) * 20 u l$ sample $=0.1 \mathrm{ug}=\mathbf{1 0 0} \mathbf{n g}$ troponin

Preparation of $10 \mathrm{ng}$ troponin solution used in the final ELISA test:

Measure out $10 \mu \mathrm{l}$ of the $100 \mathrm{ng}$ troponin solution

$\left(0.005 \frac{u g}{u l}(\right.$ trop nin solution $\left.)\right) * 10 u l$ sample $=0.05$ ug troponin

Add $90 \mu l$ of DI water

$\left(\frac{0.05 u g(\text { troponin })}{(90 u l(\text { DI water })+10 u l(\text { troponin }))}\right)=0.0005 \frac{u g}{u l}$ troponin solution

Pipette $20 \mu \mathrm{l}$ of this troponin solution onto each detection region

$\left(0.0005 \frac{u g}{u l}(\right.$ troponon solution $\left.)\right) * 20 u l$ sample $=0.01 u g=10$ ng troponin 